\title{
Roles of HDAC3-Orchestrated Circadian Clock \\ Gene Oscillations in Diabetic Rats Following Myocardial Ischemia/Reperfusion Injury
}

\section{Zhen Qiu}

Wuhan University Renmin Hospital

Hao Ming

Wuhan University Renmin Hospital

Shaoqing Lei

Wuhan University Renmin Hospital

Bin Zhou

Wuhan University Renmin Hospital

Bo Zhao

Wuhan University Renmin Hospital

Yanli Yu

Wuhan University Renmin Hospital

Rui Xue

Wuhan University Renmin Hospital

Zhong-yuan Xia ( $\nabla$ xiazhongyuan2005@aliyun.com )

Wuhan University Renmin Hospital

Original investigation

Keywords: HDAC3, circadian clock gene oscillations, mitophagy, myocardial ischemia/reperfusion injury

Posted Date: April 22nd, 2020

DOI: https://doi.org/10.21203/rs.3.rs-20879/v1

License: (1) (1) This work is licensed under a Creative Commons Attribution 4.0 International License.

Read Full License 


\title{
Roles of HDAC3-Orchestrated Circadian Clock Gene Oscillations in Diabetic Rats Following Myocardial Ischemia/Reperfusion Injury
}

\author{
Zhen Qiu ${ }^{1}$, Hao Ming ${ }^{1}$, Shaoqing Lei ${ }^{1}$, Bin Zhou ${ }^{1}$, \\ Bo Zhao ${ }^{1}$, Yanli Yu ${ }^{1}$, Rui Xue ${ }^{2}$, Zhong-yuan Xia ${ }^{1 *}$ \\ ${ }^{1}$ Department of Anesthesiology, Renmin Hospital of Wuhan University, Wuhan, Hubei 430060, China \\ ${ }^{2}$ Department of Anesthesiology, Renmin Hospital, Hubei University of Medicine, Shiyan, Hubei \\ 442000, P.R.China \\ Correspondence should be addressed to Zhong-yuan Xia; xiazhongyuan2005@aliyun.com
}

\begin{abstract}
Background: Circadian clock has been closely related to the development of diabetes mellitus and cardiovascular disease; the disruption of circadian clock exacerbates myocardial ischemia/reperfusion injury (MI/RI). HDAC3 is a key component of the circadian negative feedback loop by recruitmenting the expression pattern of circadian nuclear receptor Rev-erb $\alpha$, then to maintain the stability of circadian gene such as BMAL1. In this research, we explored the mechanism of HDAC3-orchestrated Rev-erb $\alpha /$ BMAL1 pathway in increasing MI/RI vulnerability of diabetes, and its relationship with mitophagy.
\end{abstract}

Methods and results: Streptozocin (STZ) was used to establish type 1 diabetes by intraperitoneal injection. After 8 weeks, the diabetic and non-diabetic rats were exposed to MI/RI by ligating the left anterior descending coronary artery (LDA) for 30 minutes and reperfusion for 120 minutes at four time points of zeitgeber (ZT) 0, 6, 12 and 18. Circadian clock gene oscillations were rapidly attenuated in diabetic I/RI hearts, versus sham and/or the non-diabetic I/RI hearts, in accord with circadian mitophagy. By utilizing AAV-HDAC3 to knockdown HDAC3 expression, HDAC3 deficiency significantly attenuated diabetic MI/RI associated with increased autophagy activation. In vitro experiments, primary cardiomyocytes with or without HDAC3 siRNA and Rev-erb $\alpha$ siRNA exposed to hypoxia/reoxygenation (H/R). The expression of HDAC3 and Rev-erb $\alpha$ in cardiomyocytes was increased under high glucose condition with decreased BMAL1 expression and autophagy level. After H/R stimulation, the cell injury was obviously increased with upregulated HDAC3 and Rev-erb $\alpha$ expression and decreased BMAL1 level. Moreover, high glucose aggravated H/R injury compared with low glucose by reducing autophagy level, increasing the levels of HDAC3 and Rev-erb $\alpha$ and down-regulating BMAL1 expression. HDAC3 and Rev-erb $\alpha$ siRNA can alleviate high glucose and $\mathrm{H} / \mathrm{R}$-induced injury by up-regulating BMAL1 expression and mitophagy levels.

Conclusion: These findings suggest that in diabetic rat MI/RI, the circadian clocks were rapidly impaired then to inhibit mitophagy; cardiac-targeted manipulation of HDAC3-orchestrated Rev-erba/BMAL1 pathway may be the mechanism of the decreased toleration to ischemia with a circadian dependent variability.

Keywords: HDAC3, circadian clock gene oscillations, mitophagy, myocardial ischemia/reperfusion injury 


\section{Background}

Diabetes mellitus is a major human health challenge with continuously increasing prevalence, currently estimated $1 / 8$ population of worldwide, and there will be 640 million diabetic patients in world in 2040 [1]. Diabetes and its complications are a serious threat to human health. Ischemic heart disease is the main cardiovascular complication of diabetes and the leading cause of death [2]. Therefore, the prophylaxis and treatment of cardiovascular complications of diabetes is a hot spot and difficulty for global health organization. Increasing evidences including our previous study have shown that the incidence of myocardial ischemia in diabetes is not only obviously higher than non-diabetes, but also more susceptible to myocardial ischemia/reperfusion injury (MI/RI) with increased infarct size and higher new congestive heart failure rate than non-diabetes [3-6]. However, the specific mechanisms of diabetes aggravating MI/RI are not clear, and diabetic MI/RI remain inadequately managed and the efficacy of targeted measures need to be improved. Therefore, further clarification of the pathological mechanism of diabetic MI/RI and more effective prevention measures to improve the prognosis are important issues to be solved in the current relevant disciplines.

Circadian clock is an important endogenous regulation mechanism widely existed in living organisms. The circadian clock gene oscillations play an important regulated role in diabetes, cardiovascular physiology and pathophysiology [7-8]. In the type 1 diabetes rat model, the expression of core clock genes (Rev-erb $\alpha$, BMAL1) and output genes (dbp, hlf) showed a significant phase shift, which was manifested by the advancement of the rhythm [9]. What's more, recent research has indicated that the intrinsic circadian rhythm of cardiomyocytes may contribute to the time of day dependence of cardiovascular physiology. The onset of myocardial infarction also exhibit a marked circadian rhythm in humans, with increased incidence in the early hours of the morning than in the evening [10]. Perioperative myocardial injury of the patients who undergoing aortic valve replacement is transcriptional close regulated by circadian clock [11]. Ronald Reiter et al reported that there is a circadian dependence on the time of day onset of ischemia in myocardial infarct size and left-ventricular function following STEMI [12].

As a member of class I HDAC family, HDAC3 is a important mediator in glucose metabolism and fat metabolism, and also plays an key role in the circadian rhythm negative feedback loop. The activation of HDAC3 is essential for maintaining normal circadian rhythm metabolism physiology [13]. HDAC3 is required for the inhibition of Rev-erb $\alpha$ activity. The Rev-erb $\alpha$ gene regulates the circadian rhythm to bind site in the BMAL1 gene promoter. The expression of BMAL1 protein is negatively correlated with the mRNA level of Rev-erba [14-15]. On the other hand, HDAC3 activity contributes to ischemic cardiac damage [16]. Studies have indicated that injection of HDAC3 inhibitor before or during reperfusion can reduce infarct size and maintain cardiac systolic function [17]. Circadian clock gene oscillations (i.e. Rev-erb $\alpha$, BMAL1) were rapidly attenuated in I/R, versus the non-ischemic region and sham group [18]. The amplitude of circadian clock genes (Rev-erb $\alpha$, BMAL1) oscillation in the ischemic region is rapidly attenuated, and the synchrony of circadian clock in the ischemic and non-ischemic regions exacerbates MI/RI [19-20]. Knockout or inhibition of Rev-erb $\alpha$ gene reduced myocardial damage in a sleep-awake mouse model of I/R injury [11].

Mitophagy is a key endogenous protective mechanism that maintains complete mitochondrial network function, oxidative balance and cell survival [21]. Recent studies have indicated that 
mitophagy is rhythmically and related to clock gene oscillation whose cyclic induction may provide a novel link between clock and metabolism [22]. Mitophagy is associated with biological oscillations of autophagy genes or their related genes' mRNA levels at different stages of the regulatory network. Further studies have shown that the transcription factor CCAAT/enhanced binding protein beta $(\mathrm{C} / \mathrm{EBP} \beta)$ is a clock gene and the expression of oneself is rhythmic which acts as an important transcription factor regulating mitophagy activity. Interesting, mitophagy rhythm maintenance depends on the regulation of core clock gene BMAL1 and is associated with the activation of C/EBP $\beta$ [23-24]. In diabetic state, hyperglycemia and hyperlipidemia-induced oxidative stress increases mitophagy dysfunction [25]. Previous studies have shown that mitophagy is more severe in type 1 diabetes after $\mathrm{MI} / \mathrm{R}$ insult. The use of autophagy agonist before ischemia can partially restore the mitophagy level, thereby reducing the degree of MI/RI [6]. However, the underlying mechanisms of circadian mitophagy rhythm regulated by the clock gene oscillation loop signaling pathway and whether mitophagy rhythm disorder is an important mechanism of increased MI/RI vulnerability in diabetic state have not yet clarified.

Therefore, in this study, We explored the mechanism of HDAC3-mediated Rev-erba/BMAL1 pathway in increasing MI/RI vulnerability in diabetes, and its relationship with mitophagy rhythm. Our study findings demonstrated that HDAC3-orchestrated Rev-erba/BMAL1 to regulate mitophagy is a novel mechanism in increasing $\mathrm{I} / \mathrm{R}$ vulnerability in diabetic myocardium.

\section{Materials and Methods}

\section{Experimental animals}

SPF healthy male Sprague-Dawley (SD) rats weighing 200-220g, 6-8 weeks of age, were purchased from Beijing Vital River Laboratory Animal Technology Co., Ltd. Rats were kept at the Animal Experimental Center of Renmin Hospital of Wuhan University under constant temperature and humidity with a strick 12-h light/dark cycle regime with free access to water and food. The light time is 7 a.m-7 p.m (ZT0-ZT12), and the dark time is 7 p.m.-7 a.m (time ZT12-ZT24). Experimental protocols were implemented after being reviewed and approved by the Laboratory Animal Welfare \& Ethics Committee (IACUC) of Renmin Hospital of Wuhan University. All researchers have obtained the certificate of professional laboratory animal technology in Hubei Province.

\section{AAV9 infection}

To examine the effects of HDAC3 gene down-expression in MI/R-stimulated diabetic rats, we used recombinant adeno-associated virus serotype 9 (AAV9) vectors with a GFP promoter (HBAAV9-HDAC3 shRNA1-GFP) or HBAAV9-GFP NC which were produced by Hanbio Biotechnology Co., to knockdown HDAC3 gene expression or as control. AAV-HDAC3 was given via tail vein injection at a dose of $2 \times 10^{12} \mathrm{vg} / \mathrm{kg} 3$ weeks before I/R insult.

\section{Establishment of type 1 diabetic rat model}

After fasted 12 hours, rats were administrated by intraperitoneal injection $60 \mathrm{mg} / \mathrm{kg}$ streptozotocin (STZ) (Sigma, USA) to establish diabetes model as described previously [4-6]. The fasting blood glucose (after fasting for 6 hours) was measured after $72 \mathrm{~h}$. The hallmarks of successful establishment 
of diabetes model are blood glucose levels $\geq 16.7 \mathrm{mmol} / \mathrm{L}$ with increased consumption of food and water and increased urination of rats. After that, all rats are continue raised for 8 weeks.

\section{Experimental model of $\mathrm{MI} / \mathrm{RI}$}

The model of MI/RI was established as previously described [4-6]. Briefly, after given 1\% pentobarbital sodium formulated with normal saline by intraperitoneal injection at a dose of $60 \mathrm{mg} / \mathrm{kg}$ to anaesthetize rats, the rats received mechanical ventilation after endotracheal intubation with ECG monitoring. At the fourth intercostal space of the left subclavian midline, we opened the chest to expose the heart; then the left descending coronary artery (LAD) was occluded for 30 min followed by reperfusion for $2 \mathrm{~h}$. Sham control group rats underwent same operation without LAD ligation. The non-diabetes group $(\mathrm{N})$ and the diabetes group (D) were divided into sham operation group and $\mathrm{I} / \mathrm{R}$ group at ZT0, 6, 12, and 18 respectively.

\section{Cardiac functional assessment}

Cardiac function was monitored by animal ultrasound system with rat ultrasound to measure the left ventricular developed pressure (LVDP), EF and FS\% recorded on a polygraph (RM-6240C; Chengdu Instrument Co,. LTD, China) when rats were anaesthetized by $40 \mathrm{mg} / \mathrm{kg}$ pentobarbital sodium. The measurements of two-dimensional and M-mode echocardiographic were analysed with a GE vivid 7 high-resolution in vivo imaging system (VisualSonics, Toronto, ON, Canada).

\section{Infarct size measurement}

At the end of reperfusion, LAD was ligated again and $0.3 \%$ evans blue dye (Sigma, USA) and 1\% 2,3,5-triphenyltetrazolium chloride (TTC) (Sigma, USA) staining were used to detect myocardial infarct size. Briefly, 3\% Evans blue stain was slowly injected through the femoral vein until a clearly distinguish between the blue stained and non-blue stained areas of the myocardium, and then the heart was quickly obtained. After being frozen at $-20{ }^{\circ} \mathrm{C}$ for half an hour, it was cut into $2 \mathrm{~mm}$ thick heart slices into 4-6 slices. Slices was incubated with $1 \%$ TTC solution at $37{ }^{\circ} \mathrm{C}$ thermostatic water. Then $4 \%$ paraformaldehyde was used to fix slices for 15-20 min. The area of red, white and blue in myocardial tissue were detected with a scanner (Epson, v30, Japan), and myocardial infarct size were analyzed by an image analysis system software Image-ProPlus as described previously [4-6].

\section{Measurement of serum troponin-I, CK-MB and LDH}

Serum troponin-I, CK-MB and LDH were used to evaluate myocardial injury. After reperfusion, we used assay kits (Jiancheng, Nanjing, China) to analyze the levels of serum troponin-I, CK-MB and LDH by collecting arterial blood samples through the carotid artery.

\section{Transmission electron microscopy (TEM)}

After reperfusion, $1 \mathrm{~mm}^{3}$ tissue of the left ventricle in rats' hearts were collected and fixed with in $2.5 \%$ glutaraldehyde for $24 \mathrm{~h}$. The tissue samples were washed, fixed, dehydrated, embeded and cured with buffer solution, then were cut into ultrathin sections by an ultra-thin microtome. The TEM 
micrographs of ultrathin sections were detected by TEM (Tecnai $\mathrm{G}^{2} 20$ TWIN, USA) under the help of professional teachers of the Core Facility of Wuhan Institute of Virology.

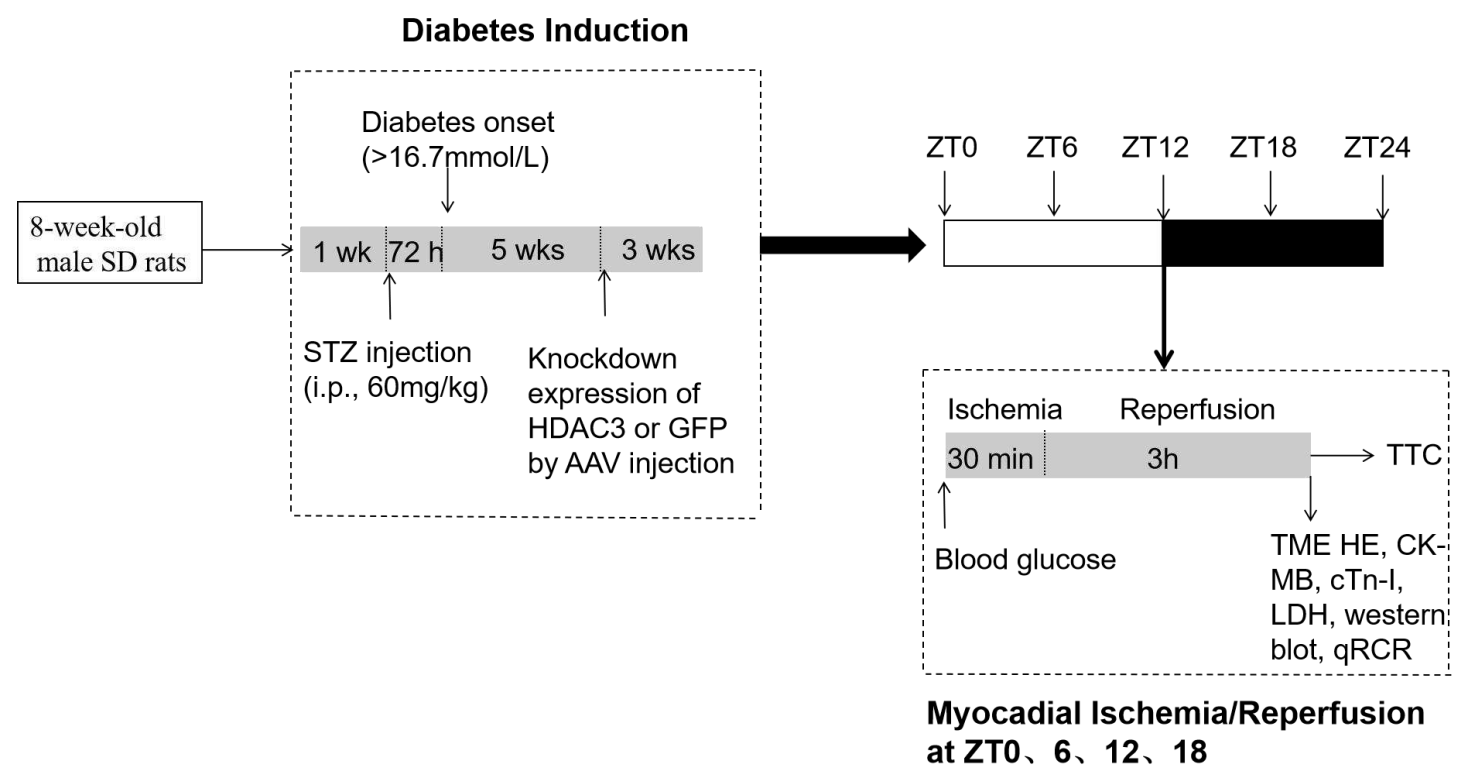

Fig. 1 Schematic diagram of experimental program. It illustrates the period of diabetic induction, the time points of adeno-associated virus injection, ischemia/reperfusion administration and data acquisition.

\section{In vitro experimental protocol and hypoxia/reoxygenation model}

Primary neonatal rat cardiomyocytes were obtained from newborn SD suckling rats at 1-3 days. Briefly, newly born SD rats were disinfected with $75 \%$ alcohol, chest open and the heart was harvest, then cut up in ADS buffer (containing $120 \mathrm{mM} \mathrm{NaCl}, 20 \mathrm{mM}$ HEPES (pH 7.4), $8 \mathrm{mM} \mathrm{NaH2PO}$, 6 $\mathrm{mM}$ glucose, $5 \mathrm{mM} \mathrm{KCL}$, and $0.8 \mathrm{mM} \mathrm{MgSO} 4$; Sinopharm Chemical Reagent co.,Ltd, China). After that, the tissue fragments were digested in ADS solution containing 1\% collagenase from clostridium histolyticum (Sigma, USA) and $0.75 \%$ pancreatin from porcine pancreas (Sigma, USA) for 6 times, 20 minutes each time at $37{ }^{\circ} \mathrm{C}, 70 \mathrm{rpm}$ in constant temperature shaker. Cell suspension was then centrifuged for $3 \mathrm{~min}$ at $2200 \mathrm{rpm}$, and the supernatant was discarded. Resuspend the obtained cell pellet into the top of the percoll gradient, centrifuge at $3000 \mathrm{rpm}$ for 30 minutes at room temperature. Collect the cardiac cells and then was centrifuged at $2200 \mathrm{rpm}$ for 3 minutes with $20 \mathrm{ml}$ ADS buffer. Then the cells were cultured in 6-well plates and 96-wells for experiments with DMEM (Gibco, USA) containing $10 \%$ fetal bovine serum (Gibco, USA) and 1\% penicillin and 1\% streptomycin in a cell culture incubator at $37^{\circ} \mathrm{C}$ within $5 \% \mathrm{CO}_{2}$. HDAC3 siRNA and Rev-erb $\alpha$ siRNA (Ribobio, China) transfections were performed by using lipofectamineTM 2000 (Invitrogen, USA). After $24 \mathrm{~h}$ post-transfection, the cells were exposed to high glucose $(\mathrm{HG})$ for $24 \mathrm{~h}$ treated at non-toxic concentrations. Subsequently, Neonatal cardiomyocytes were subjected to hypoxic conditions $(0.9 \%$ $\mathrm{O}_{2} / 94.1 \% \mathrm{~N}_{2} / 5 \% \mathrm{CO}_{2}$ ) for $6 \mathrm{~h}$, followed $2 \mathrm{~h}$ normal condition for reoxygenation.

\section{Cell viability assay}


CCK-8 assay kit (Jiancheng, Nanjing, China) was used to measure cell viability. After stimulation, the cultured cells in 96-well plates was given $10 \mu \mathrm{l}$ CCK-8 reagent for each well and then incubated for 3 $\mathrm{h}$ in darkness. Perkin Elmer Microplate reader (PerkinElmer Victor 1420, USA) was used to analyze the absorbance at $450 \mathrm{~nm}$.

\section{Mitochondrial ROS measurement}

MitoTracker Red CMXRos assays (YEASEN, China) was used to measure the mitochondrial ROS production. $500 \mathrm{nM}$ MitoTracker Red CMXRos working liquid was added to the cells and incubated with at $37{ }^{\circ} \mathrm{C}$ for $30 \mathrm{~min}$ in darkness. After that, cold PBS was used to washed cells twice. The fluorescence intensity of mitochondrial ROS was recorded by using fluorescence microscopy (Olympus IX51).

\section{Assessment of mitochondrial membrane potential (MMP)}

MMP was detected by JC-1 (Beyotime, China) staining according to manufacturer's instructions. Briefly, at the end of experimental stimulation, cells were incubated with JC-1 dye working liquid at $37^{\circ} \mathrm{C}$ for $20 \mathrm{~min}$ in darkness. Subsequently, JC-1 buffer was used to washed the cells twice. Images of cells were obtained immediately and analyzed by using a fluorescence microscope (Olympus IX51, Japan). When the mitochondrial membrane potential is high, JC-1 aggregates into the matrix of the mitochondria to form a polymer (J-aggregates), which can produce red fluorescence. When the mitochondrial membrane potential is low, JC-1 cannot aggregate mitochondria in the matrix and JC-1 is a monomer at this time, and then green fluorescence can be produced.

\section{Measurement of autophagic flux}

To measure the autophagic flux in cardiomyocytes, we used the tandem fluorescent mRFP-GFP-LC3 adenovirus $(\mathrm{MOI}=100)$ to transfect curtured cells. GFP and mRFP in mRFP-GFP-LC3 adenovirus were used to label and track LC3. $24 \mathrm{~h}$ after adenoviral transfection, cells were washed with PBS, fixed with $4 \%$ paraformaldehyde, mounted with a reagent containing DAPI (Sigma, USA). The expression of GFP and mRFP was detected with Olympus FV1200 laser scanning confocal microscope (Olympus, Japan). Attenuation of GFP indicate that lysosomes and autophagosomes fuse to form autophagosomes, which red fluorescence can only be detected at this time. Yellow (merge of GFP signal and RFP signal) puncta represented early autophagosomes, while red (RFP signal alone) puncta indicate late autolysosomes. Images were abtained by using FV10-ASW3.0 software. The autophagic flux was evaluated by counting the spots of different colors.

\section{Gene expression}

Following the manufacturer's instructions, total RNA was extracted from cells by using trizol reagent (Invitrogen, USA), then reversely transcribed $1 \mu \mathrm{g}$ total RNA into cDNA by using a reverse transcription kit (Takara, China). The mRNA levels of HDAC3, Rev-erb $\alpha$, BMAL1, CEBP/ $\beta$ were performed by quantitative RT-PCR in $20 \mu \mathrm{l}$ reaction system containing specific primers which synthesized by Sangon Biotech (Shanghai, China) and SYBR Green Master Mix (Takara, China) by Bio-Rad CFX Connect Real-Time PCR Detection System (Bio-Rad, USA). The levels of mRNA were 
normalized relative to GAPDH. The expression of genes was analyzed by using the $2^{-\triangle \triangle} \mathrm{CT}$ method.

\section{Western Blotting}

Myocardial proteins were lysed in ice-cold radio immunoprecipitation assay buffer, and then centrifuged at $12000 \mathrm{rpm}$ at $4{ }^{\circ} \mathrm{C}$ for $15 \mathrm{~min}$ to collect supernatants. Protein lysates were loaded into an 5\%-10-12\% SDS-PAGE gel and transferred to polyvinylidene difluoride (PVDF) membrane, and then incubated with specific primary antibodies at $4{ }^{\circ} \mathrm{C}$ overnight. Subsequently incubated with fluorescent secondary antibody for $1 \mathrm{~h}$ at room temperature. The protein bands were obtained by using odyssey color infrared laser scan-imaging instrument (Li-Cor, USA).

\section{Statistical Analysis}

All data are expressed as the mean \pm standard deviation. GraphPad Prism version 8.0 (GraphPad Software, USA) was used to statistical software analysis. Differences among experimental groups were analyzed by ANOVA followed by post-assay/test method by Bonferroni correction for post hoc t-test. $\mathrm{P}$ values $<0.05$ were considered to be statistically significant.

\section{Results}

\section{Circadian clock gene rhythmicity is altered in SZT-induced diabetic rats heart}

Many researches have demonstrated that circadian rhythms plays a profound influence on cardiovascular physiology in rat and other animal models [10-12]. Therefore, to explore the alteration of circadian clock gene, we examined the diurnal oscillations of Rev-erb $\alpha$, BMAL1 and C/EBP $\beta$ mRNA levels in non-diabetic and diabetic rats at the beginning of the light phase (ZT0), the middle of the light phase (ZT6), the beginning of the dark phase (ZT12) and the middle of the dark phase (ZT18). The mRNAs encoding for both of these components (Rev-erb $\alpha$, BMAL1 and C/EBP $\beta$ ) have a circadian expression pattern in hearts isolated from both non-diabetes and diabetes animals (Fig. 2). The expression of Rev-erb $\alpha$ gene in diabetic heart was much higher than in non-diabetic with attenuated amplitude (Fig. 2a). The diurnal oscillations of clock gene BMAL1 were significantly attenuated in diabetic hearts under lower level (Fig. 2b). On the other hand, time-dependent up-regulation of C/EBP $\beta$ was observed in diabetic hearts compared with non-diabetic rats (Fig. 2c). Collectively, these data suggest that the genetic disruption of cardiomyocyte circadian clock altered the mRNA levels of Rev-erb $\alpha$, BMAL1 and $\mathrm{C} / \mathrm{EBP} \beta$ in myocardial tissue of diabetic heart, which implies a potential role in diabetic cardiomyopathy. 

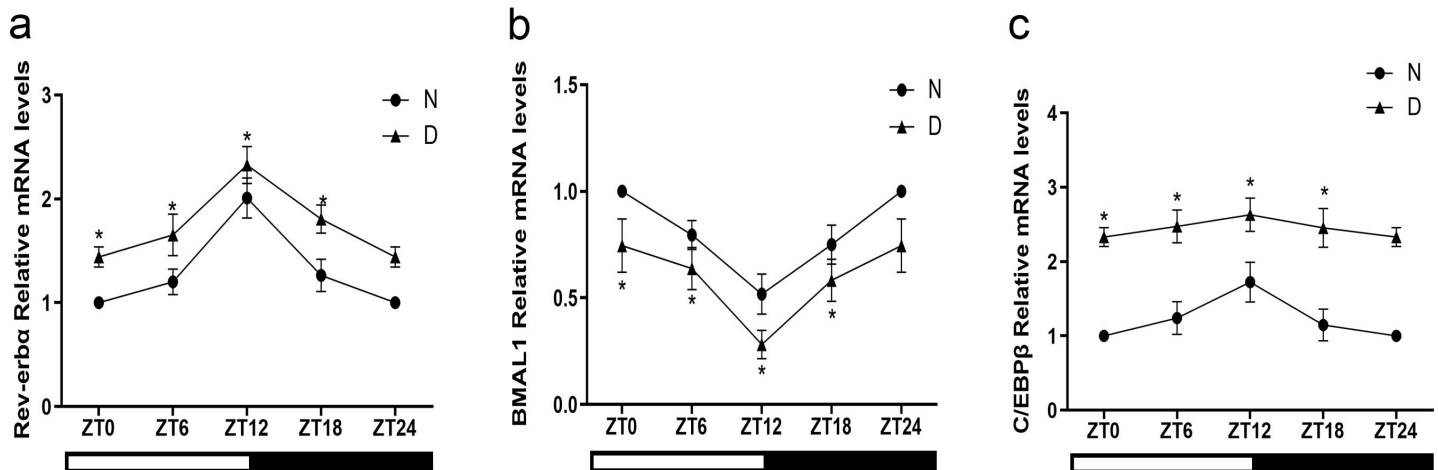

Fig. 2 Rev-erb $\alpha$, BMAL1 and C/EBP $\beta$ mRNA diurnal variations in non-diabetic rats (NS) and diabetic rats (DM). (a-c) Expression levels of Rev-erb $\alpha$ (a), BMAL1 (b) and C/EBP $\beta$ (c) were measured by qPCR over time after reperfusion. $\mathrm{n}=6$ per group. ${ }^{*} \mathrm{P}<0.05$ versus $\mathrm{N}$ within $\mathrm{ZT}$. $\mathrm{N}$, non-diabetes; $\mathrm{D}$, diabetes.

\section{Determination of the circadian dependence of $\mathrm{MI} / \mathrm{RI}$ in diabetic and non-diabetic rats}

To investigate the relationship between time-of-day-dependent oscillations in $M I / R$ tolerance and circadian rhythm under diabetes condition, we subjected diabetic and non-diabetic rats to ischemia/reperfusion stimulation at ZT0, ZT6, ZT12 and ZT18. As shown in Fig. 3a, we observed the diurnal variations of infarct size in non-diabetic and diabetic rats. As predicted, maximal injury was observed at the time of ZT12 shown by infarct size in non-diabetic rats than other time, which is consistent with previous reports [11]. More importantly, the infarct size of diabetic hearts following MI/R at both ZT0, ZT6, ZT12 and ZT18 was significant increased than in non-diabetic rats and there is no difference between these points. The time-of-day-dependence of infarct size in non-diabetic was accompanied by the diurnal variations of cTn-I, CK-MB and LDH following MI/R. Fig. 3b-d shows that the levels of cTn-I, CK-MB and LDH were increased rapidly with attenuation/abolition of diurnal variations in diabetic $\mathrm{I} / \mathrm{R}$ hearts compared with non-diabetic $\mathrm{I} / \mathrm{R}$ hearts at different point. In addition, these parameters peak after ischemic insult is performed at ZT12 compared with ZT0. 

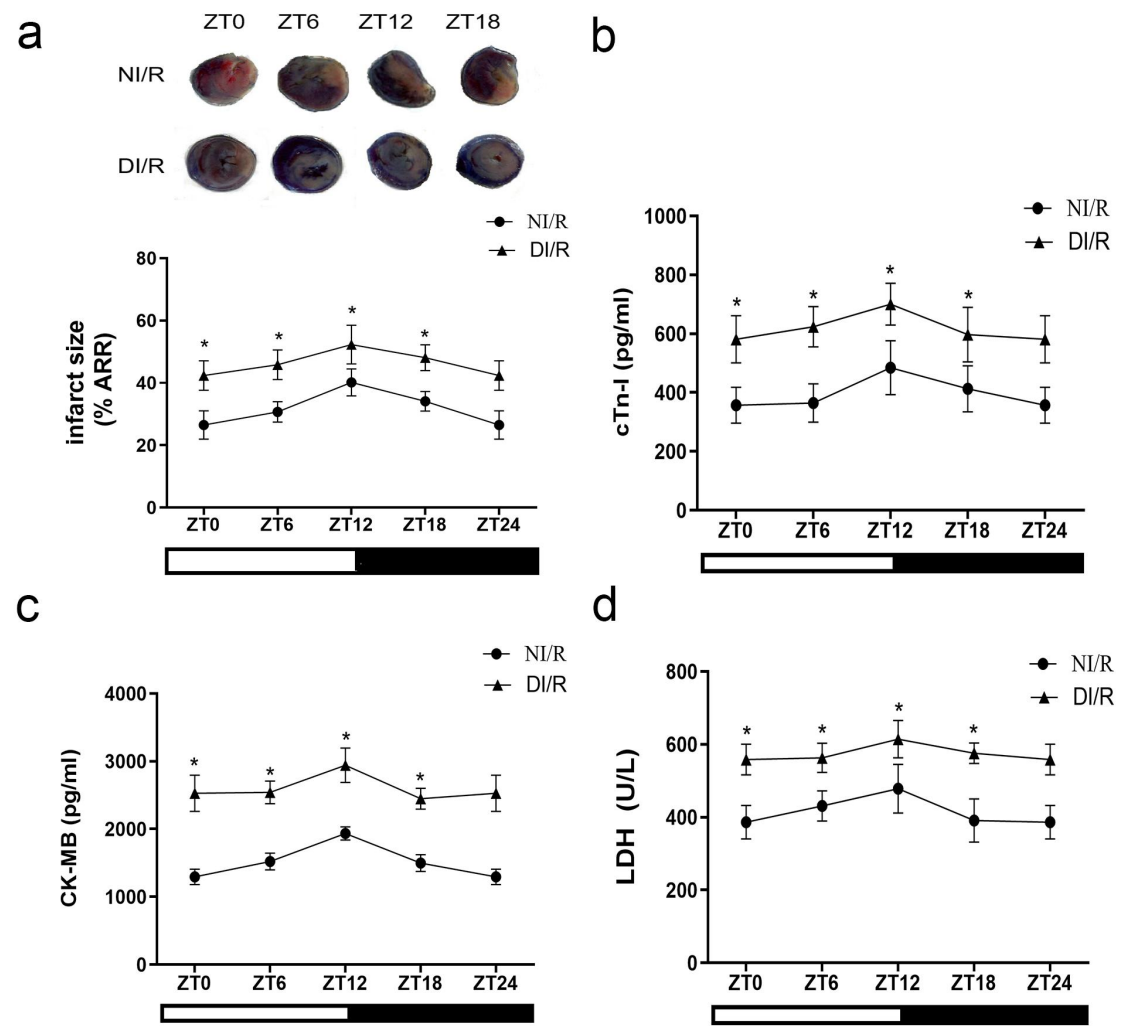

Fig. 3 Infarct size, levels of cTn-I, CK-MB and LDH diurnal variations are dependent on time-of day of MI/RI in diabetic and non-diabetic rats. (a) Infarct size was detected by TTC. (b-d) The levels of cTn-I (b), CK-MB (c) and LDH (d) was detected by ELSAL in the surum of non-diabetes and diabetes with or without I/R insult. Scale bar: $2 \mathrm{~mm}$. $\mathrm{n}=6$ per group. ${ }^{*} P<0.05$ versus $\mathrm{NI} / \mathrm{R}$ within $\mathrm{ZT}$. NI/R, non-diabetes ischemia/reperfusion; DI/R, diabetes ischemia/reperfusion.

\section{Mitophagy is impaired and rhythm disordered at circadian dependent in diabetes after MI/RI}

To determine the activity and rhythm of mitophagy after MI/RI in diabetic and non-diabetic rats, we next used TEM to detect mitochondrial damage, autophagosome and autolysosome counts in rat heart at time-of-day-specific points after MI/RI. We found that the counts of autopahgosomes and autolysosomes shown rough oscillations, minimum at ZT12 in non-diabetic rat (Fig. 4a-b). Compared with non-diabetic rats, the mitochondrial damage is more serious in diabetic rats after MI/RI, and the autophagosomes significantly reduced with no rhythmic fluctuation at different times.

Next, we examined protein expression patterns of mitophagy genes by western blotting. As shown in Fig. 4c-j, in accordance with the changes of autophagosomes and autolysosomes, mitophagy genes C/EBP $\beta$ (Fig. 4c) and BNIP3 (Fig. 4e) peak at ZT12 and Atg4b (Fig. 4d) and LC3II/I (Fig. 4f) minimum at ZT12 with circadian dependent rhythm in non-diabetes sham and I/R group. However, the expression of genes and proteins have shown rhythm disorder at circadian dependent in diabetes with or without $\mathrm{I} / \mathrm{R}$ stimulation. On the other hand, compared with sham group, the protein levels of C/EBP $\beta$ (Fig. 4c) and BNIP3 (Fig. 4e) were significant increased in $\mathrm{I} / \mathrm{R}$ group of non-diabetic or diabetic rats. These data implicated that circadian mitophagy rhythm and circadian of autophagy genes 
in heart is an vital factor in mitochondria function of myocardial, and the mitochondrial damage and mitophagy rhythm disorder in diabetes may be the mechanism of increased vulnerability of MI/RI.

a

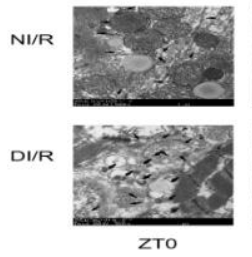

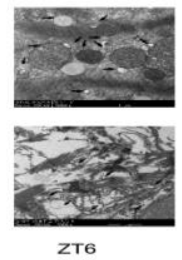

ZT6
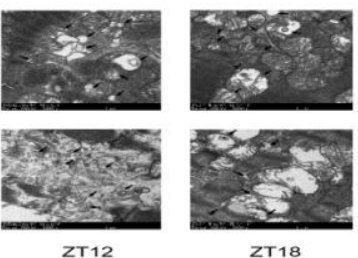

b

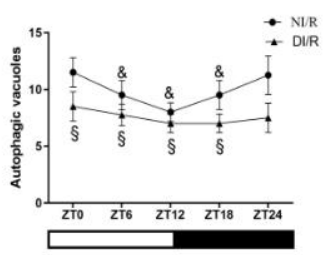

C

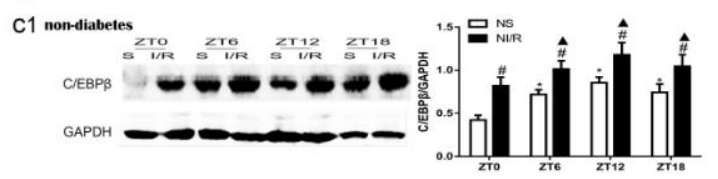

C2

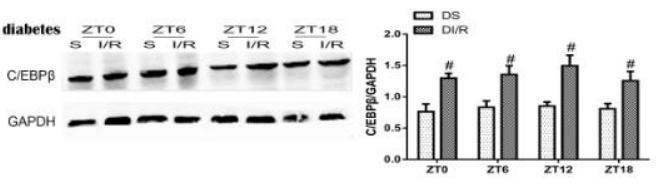

e

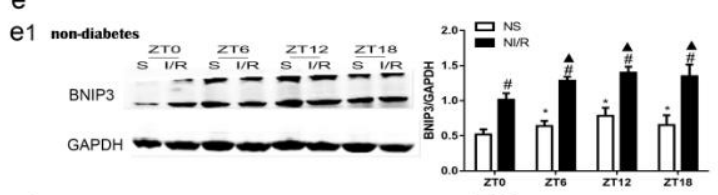

e2

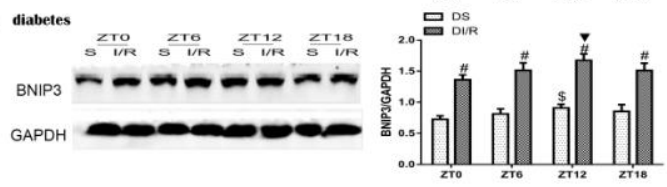

d

d1 non-diabetes

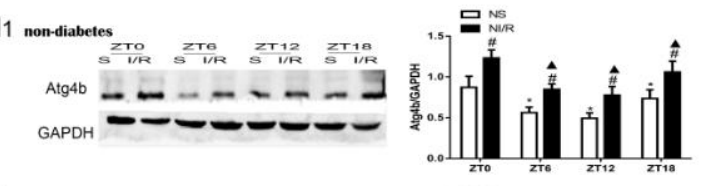

d2 diabet

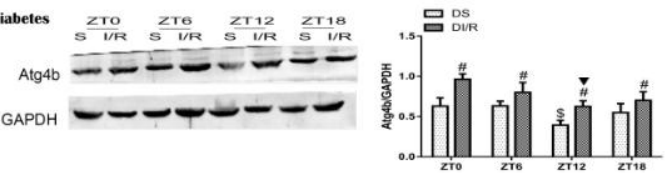

f

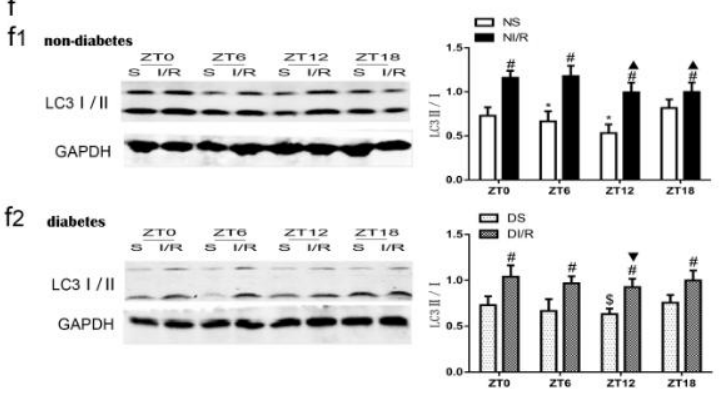

Fig. 4 Mitochondrial damage, autophagosome, and the proteins expression of mitophagy-related genes diurnal variations are dependent on time-of day of MI/RI in non-diabetic and diabetic rats. (a-b) The ultrastructural changes and autophagic vacuoles of rat hearts were detected by TEM, Scale bar: $1 \mu \mathrm{m}$. (c-f) The protein levels of C/EBP $\beta$ (c), Atg4b (d), BNIP3(e) and LC3II/I (f) was detected by western blotting in the myocardial tissues of non-diabetes and diabetes with or without I/R insult. $\mathrm{n}=6$ per group. ${ }^{\S} P<0.05$ versus NI/R within $Z$ T; ${ }^{\&} P<0.05$ versus ZT0 within NI/R; ${ }^{*} P<0.05$ versus ZT0 within NS; ${ }^{\#} P<0.05$ versus sham within $Z \mathrm{~T}$; ${ }^{\$} P<0.05$ versus $Z \mathrm{~T} 0$ within $\mathrm{DS}$; ${ }^{\star} P<0.05$ versus $Z \mathrm{~T} 0$ within NI/R; ${ }^{\nabla} P<0.05$ versus ZT0 within DI/R. NS, non-diabetes sham; NI/R, non-diabetes ischemia/reperfusion; DS, diabetes sham; DI/R, diabetes ischemia/reperfusion.

\section{HDAC3 mediated circadian gene expression oscillations disrupted in diabetic $M I / R$ rats to increased the injury}

Previously published studies suggest that HDAC3 is an essential component for maintaining normal circadian rhythm metabolism physiology and an important role in MI/RI [18,21]. Given the known central role of circadian gene in modulating MI/R-induced myocardial damage. We next examined the gene and protein expression of HDAC3, Rev-erba and BMAL1. As shown in Fig. 5a and c, the expression of HDAC3 was not significantly rhythmic after I/R stimulated in non-diabetes and diabetes; in the same time point, the mRNA and protein levels of HDAC3 were significant increased in DI/R 
group than in NI/R group. As shown in Fig. 5b, Rev-erba mRNA level showed different oscillation amplitude both in non-diabetic and diabetic rats after $\mathrm{I} / \mathrm{R}$ insult, and amplitude in diabetic rats was significantly lower and dislocated than that in non-diabetic rats; the mRNA level of Rev-erbo in diabetic rats were significantly higher than in non-diabetic rats after MI/RI. As shown in Fig. 5e, in non-diabetic or diabetic rats, the level of Rev-erb $\alpha$ protein after $\mathrm{I} / \mathrm{R}$ was significantly higher than that of sham operation groups; showed significant rhythms at different time points. In NI/R groups, the expression level of Rev-erb $\alpha$ protein peaked at ZT12. In diabetic rats, Rev-erb $\alpha$ protein expression levels in sham operation and I/R groups were decreased rhythmic amplitude (Fig. 5e). In condition, the expressions of BMAL1 (Fig. 5c and f) showed different amplitudes of oscillation in non-diabetes and diabetes rats with or without $\mathrm{I} / \mathrm{R}$ insult, and amplitude in diabetic rats was significantly lower and dislocated than that in non-diabetic rats; and the BMAL1 expression shows the lowest level at ZT12 in $\mathrm{NI} / \mathrm{R}$ groups. What's more, in the same time point, compared with NI/R group, the mRNA and protein levels of BMAL1 were significant decreased in DI/R group showing in Fig. 5.

a

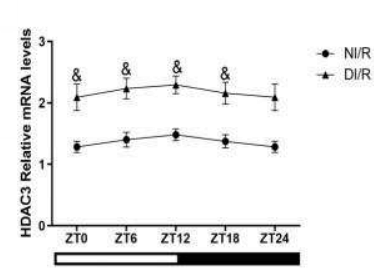

d

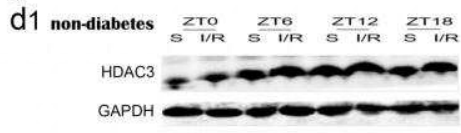

d2
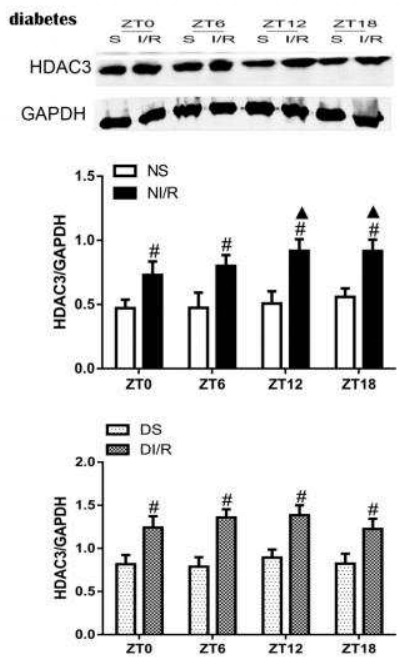

b

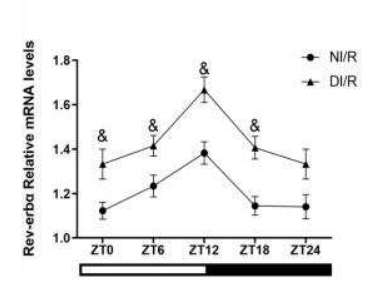

e

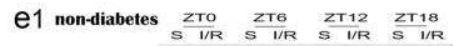

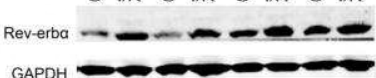

e2
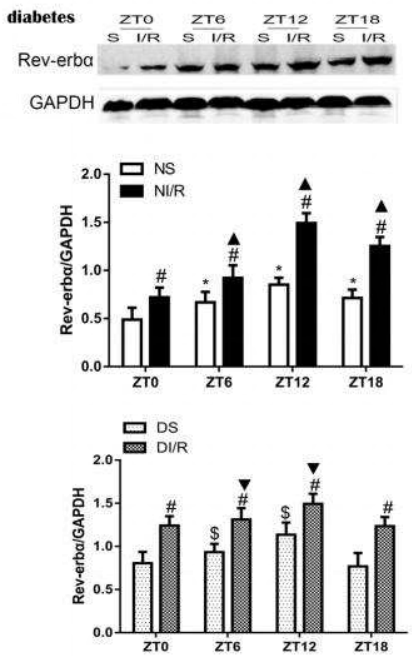

C

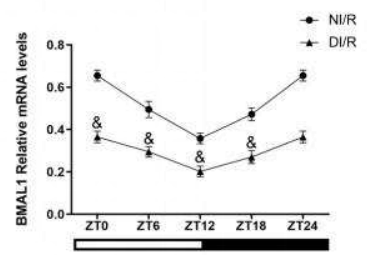

\section{f}

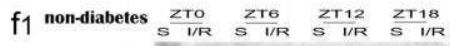
BMAL1 $0-0-\cdots-$ GAPDH

$\mathrm{f} 2$
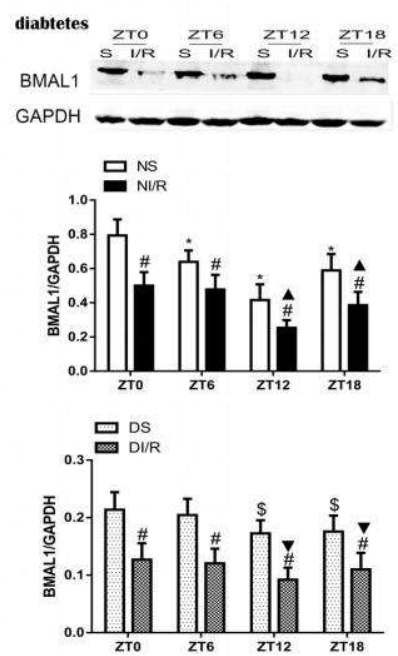

Fig. 5 The mRNA and protein expression of HDAC3 and circadian genes (Rev-erb $\alpha$ and BMAL1) are dependent on time-of day of MI/RI in non-diabetes and diabetes. (a-c) The mRNA levels of HDAC3 (a), Rev-erb $\alpha$ (b) and BMAL1 (c) were analysed by qPCR. (d-f) The protein levels of HDAC3 (d), Rev-erb $\alpha$ (e) and BMAL1 (f) was analysed by western blotting in myocadial tissues of non-diabetes and diabetes with or without $\mathrm{MI} / \mathrm{R}$ insult. $\mathrm{n}=6$ per group. ${ }^{\&} P<0.05$ versus NI/R within ZT; ${ }^{*} P<0.05$ versus ZT0 within NS; ${ }^{\#} P<0.05$ versus sham within $Z \mathrm{~T} ;{ }^{\$} P<0.05$ versus $Z \mathrm{~T} 0$ within DS; ${ }^{\boldsymbol{}}$ 
$P<0.05$ versus ZT0 within NI/R; ${ }^{\nabla} P<0.05$ versus ZT0 within DI/R. NS, non-diabetes sham; NI/R, non-diabetes ischemia/reperfusion; DS, diabetes sham; DI/R, diabetes ischemia/reperfusion.

\section{Up-regulation of HDAC3-mediated Rev-erba/BMAL1 pathway increase the vulnerability to MI/RI by inhibiting mitophagy in diabetes}

Our previous studies have shown that $\mathrm{MI} / \mathrm{RI}$ is more grievous in diabetes than in non-diabetes, and its mechanism is related to the decreased autophagy level in diabetic rats [5-6], but the specific mechanism needs further study. So, in the next, we investigate whether HDAC3-regulated circadian gene mediate autophagy activation is involved in the more serious MI/RI of diabetic rats at ZT12 time point which is the most severe time point of MI/RI. As shown in Fig. 6a, after 8 weeks of diabetes establishment, echocardiography showed an decreased left ventricular ejection fraction (LVEF) and left ventricular fractional shortening (LVFS) in diabetic rats than in non-diabetic rats. After I/R stimulation at ZT12, the protein levels of HDAC3 (Fig. 6b) and Rev-erb $\alpha$ (Fig. 6c) were increased in DI/R group compared with NI/R group; the level of BMAL1 (Fig. 6d) was obviously decreased in DS group than in NS group and $\mathrm{I} / \mathrm{R}$ insult further decreased the level of BMAL1. What's more, the expression of autophagy proteins C/EBP $\beta$ (Fig. 6e), BNIP3 (Fig. 6g), Atg4b (Fig. 6f) and LC3II/I (Fig. 6h) was obviously increased after MI/RI than in sham-operated groups of non-diabetes and diabetes. However, the levels of Atg4b and LC3II/I in diabetes were prominently decreased than that in non-diabetic rats, and the increase degree of autophagy protein was significantly lower in diabetes than in non-diabetes after MI/RI . 
a

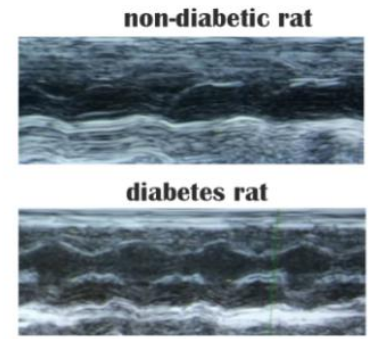

C
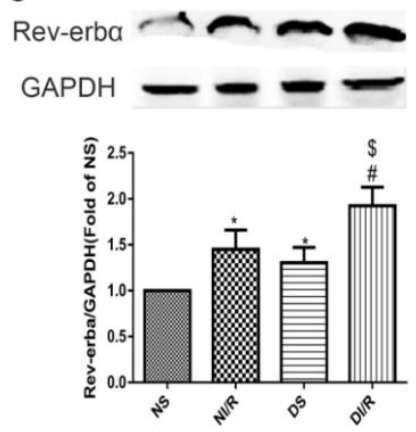

f
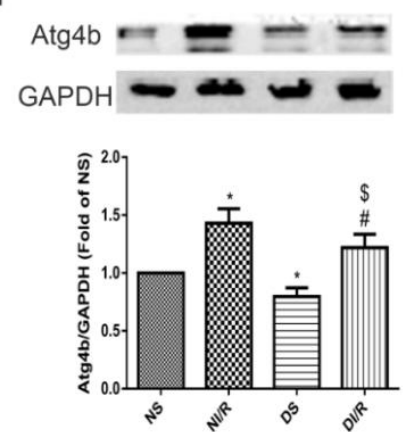

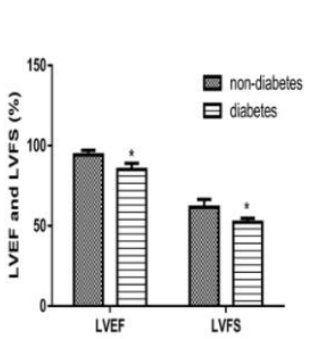

b

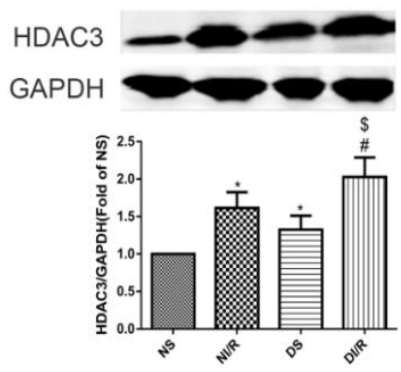

d
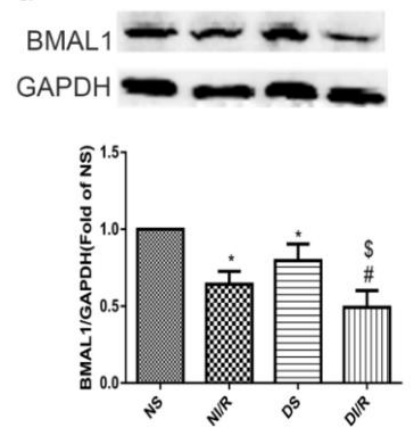

e
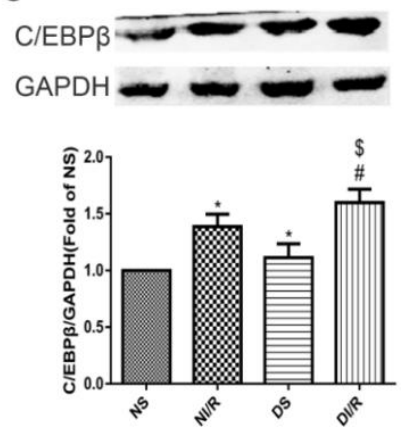

g

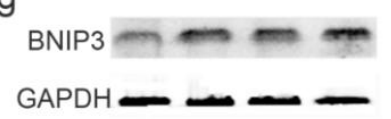

h
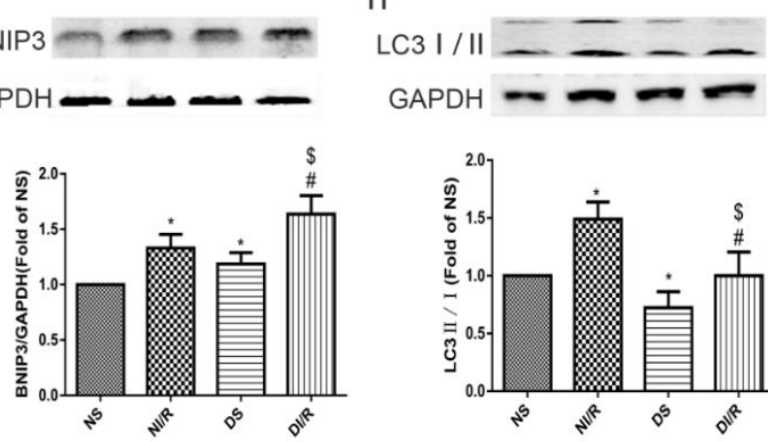

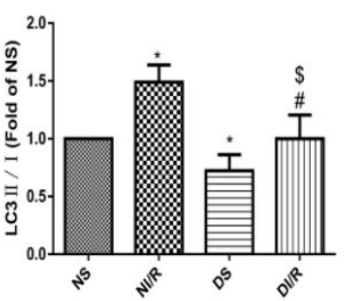

Fig. 6 HDAC3 up-regulated expression promotes cardiac dysfunction and down-regulates autophagy by mediated circadian genes in diabetic rats to aggravate MI/RI. (a) Cardiac function was assessed by echocardiography in non-diabetic and diabetic rats. (b-h) The protein levels of HDAC3 (b), Rev-erb $\alpha$ (c), BMAL1 (d), C/EBP $\beta$ (e), Atg4b (f), BNIP3(g) and LC3II/I (h) was detected by western blotting. Scale bar: $2 \mathrm{~mm}$. $\mathrm{n}=6$ per group. ${ }^{*} P<0.05$ versus NS; ${ }^{\#} P<0.05$ versus $\mathrm{DS} ;{ }^{\$} P<0.05$ versus NI/R. NS, non-diabetes sham; NI/R, non-diabetes ischemia/reperfusion; DS, diabetes sham; DI/R, diabetes ischemia/reperfusion.

\section{Knockdown HDAC3 expression attenuated MI/RI in diabetes}

Up-regulation of HDAC3 in MI/RI implied an vital role in the injury response. Next, we determined the mechanism of hyperglycaemia-induced HDAC3 up-regulation aggravated MI/RI in diabetic rats. So we knockdown the HDAC3 protein expression via HBAAV9-r-HDAC3 shRNA1-GFP injections in myocardial tissue of diabetic rats. 3 weeks after HBAAV9-r-HDAC3 shRNA1-GFP and HBAAV9-GFP NC infection, we found that the GFP signals were clearly visible, which indicated successful transfection and strong expression of AAV-GFP-NC and AAV-GFP-HDAC3 (Fig. 7a). A 
similar pattern of expression was evident by HDAC3 protein. Compared with DM control group and AAV-GFP transfection, AAV-HDAC3 transfection greatly decreased HDAC3 protein levels (Fig. 7b). As shown in Fig. 7c, down-regulation of HDAC3 significant reduced infarct size compared to control group after ischemia for $30 \mathrm{~min}$ and reperfusion for 2 hours. Furthermore, as the key biomarkers for cardiac injury, cTn-I (Fig. 7d), CK-MB (Fig. 7e) and LDH (Fig. 7f) was also obviously blunted in AAV-HDAC3 group.
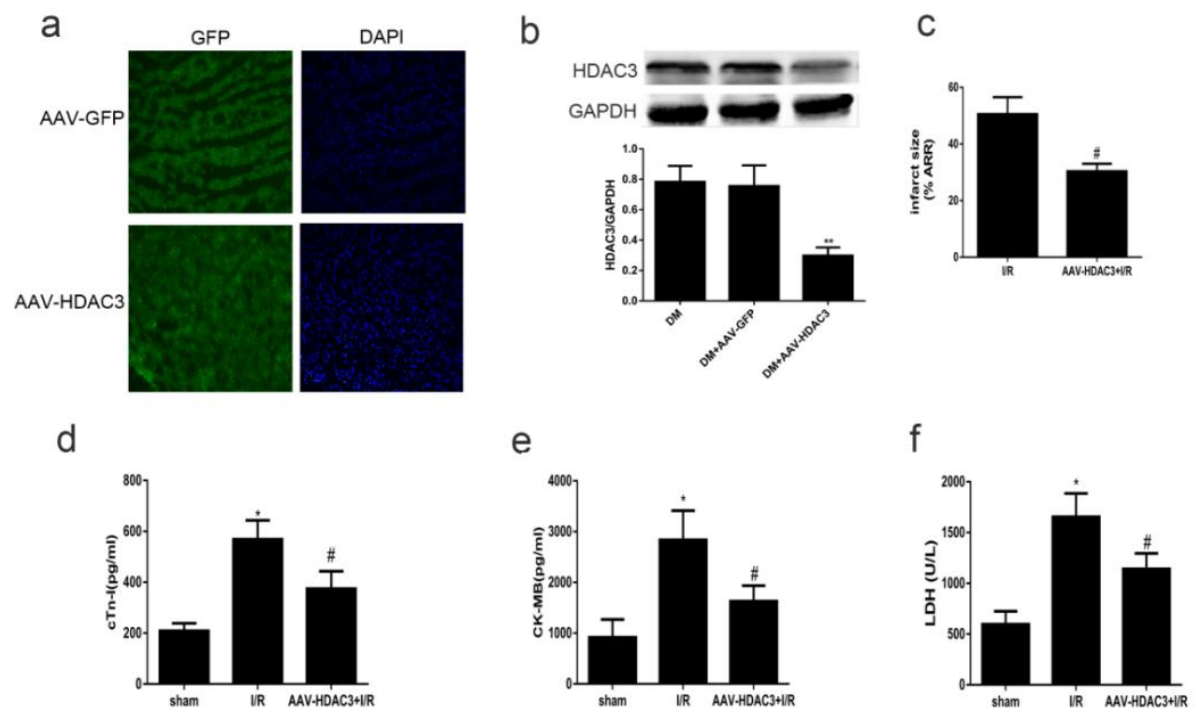

Fig. 7 Knockdown expression of HDAC3 attenuated MI/RI of diabetes. (a) Representative images of immunofluorescence staining for GFP and DAPI in myocardial tissue after AAV-GFP and AAV-HDAC3 transfection. (b) Protein expression of HDAC3 was analyed by western blotting. (c) Infarct size was measured by TTC. (d-f) The serum levels of cTn-I (d), CK-MB (e) and LDH (f) were measured by ELISA assay kits. scale bar $=100 \mu \mathrm{m}$. $\mathrm{n}=6 \mathrm{per}$ group. ${ }^{* *} P<0.01$ versus $\mathrm{DM} ;{ }^{*} P<0.05$ versus sham; ${ }^{\#} P<0.05$ versus I/R. I/R, ischemia/reperfusion; AAV-HDAC3+I/R, HBAAV9-r-HDAC3 shRNA1-GFP injection+ ischemia/reperfusion.

\section{Genetic knockdown of HDAC3 significantly attenuates diabetic MI/RI by mediating Rev-erbo/BMAL1 circadian pathway to recover mitophagy}

To explore the role of knockdown HDAC3 expression in circadian oscillations and autophagy in the $\mathrm{MI} / \mathrm{RI}$ of diabetic rats, we next assayed the mRNA expression of circadian gene, and the proteins expression of circadian genes and autophagy-related gene. As expected, the mRNA level of HDAC3 (Fig. 8a) in I/R group was obviously increased than sham group of diabetic rats with the same result in Rev-erb $\alpha$ mRNA and protein level (Fig. 8b and d). However, the tendency was reversed notably by HDAC3 down-expression. In addition, the mRNA and protein level of BMAL1 (Fig. 8c and e) were significant increased in AAV-HDAC3 $+\mathrm{I} / \mathrm{R}$ group than $\mathrm{I} / \mathrm{R}$ group in diabetes. Western blotting showed that the expression of autophagy proteins C/EBP $\beta$ (Fig. 8f), BNIP3 (Fig. 8g) and LC3 II/I (Fig. 8i) increased significantly after MI/RI compared with the sham group with decreased P62 expression (Fig. 8h). MI/RI following AAV-HDAC3 transfection showed obvious increased LC3II/I level and 
decreased P62 expression. What's more, compared with $\mathrm{I} / \mathrm{R}$ group in diabetes, AAV-HDAC3 transfection significant reversed the increased protein levels of $C / E B P \beta$ and BNIP3.

a

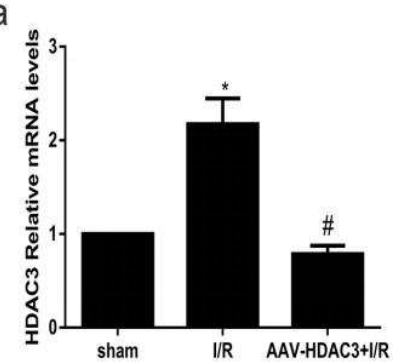

d
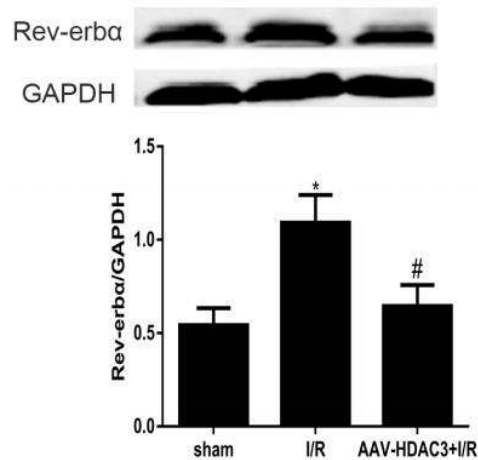

g
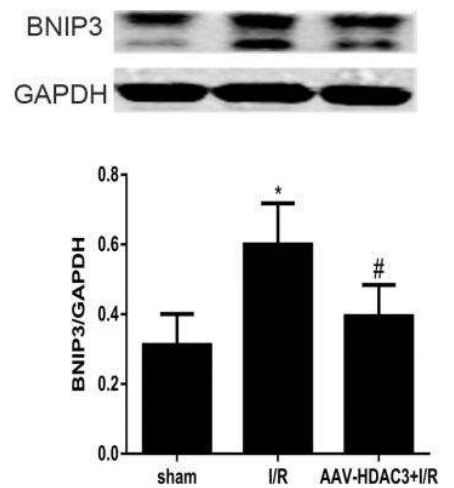

b

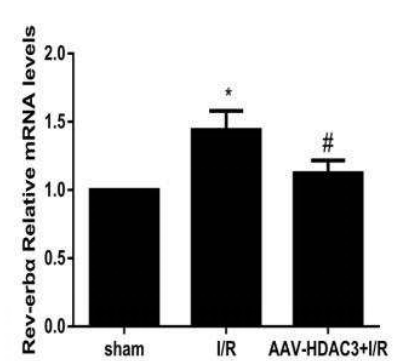

e
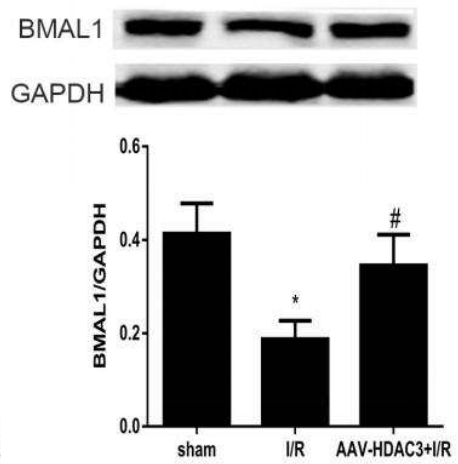

h
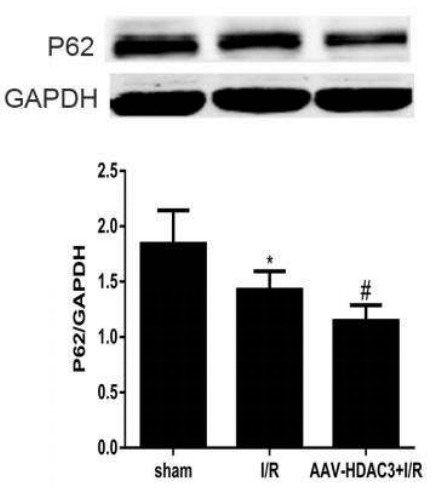

C

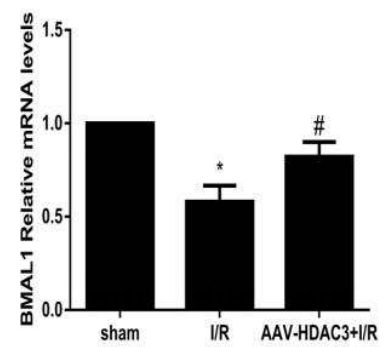

f

C/EBP $\beta$

GAPDH

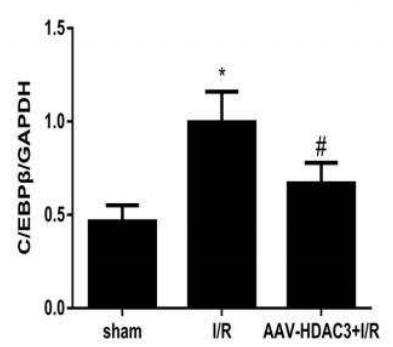

i
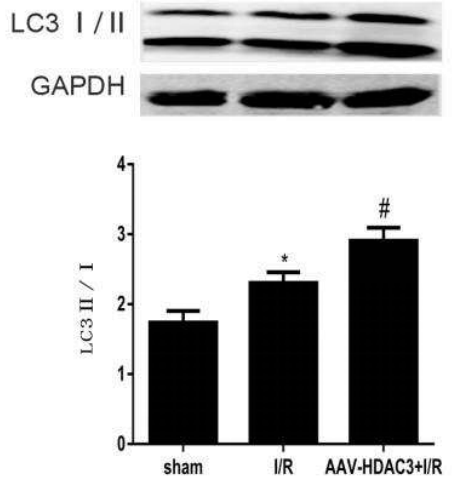

Fig. 8 Knockdown expression of HDAC3 attenuated diabetic MI/RI by regulating Rev-erb $\alpha /$ BMAL1 circadian oscillations to recover mitophagy level. (a-c) The mRNA expressions of HDAC3 (a), Rev-erb $\alpha$ (b) and BMAL1 (c) were analysed by qPCR. (d-i) The protein levels of Rev-erb $\alpha$ (d), BMAL1 (e), C/EBP $\beta$ (f), BNIP3(g), P62 (h) and LC3II/I (i) were measured by western blotting in non-diabetes and diabetes with or without I/R insult. $n=6$ per group. ${ }^{*} P<0.05$ versus sham; ${ }^{*} P<0.05$ versus $\mathrm{I} / \mathrm{R}$. I/R， ischemia/reperfusion; AAV-HDAC3+I/R， HBAAV9-r-HDAC3 shRNA1-GFP injection+ ischemia/reperfusion.

High glucose aggravates $H / R$ injury of neonatal rat cardiomyocytes by reducing autophagic flow levels and mitochondrial function 
As shown in Fig. 9a, HG could significantly decrease cell viability than LG which was further aggravated by H/R stimulation. The level of LDH release (Fig. $9 \mathrm{~b}$ ) in neonatal rat cardiomyocytes was increased in $\mathrm{HG}$ condition and further increased by $\mathrm{H} / \mathrm{R}$ stimulation. Moreover, $\mathrm{HG}$ and $\mathrm{H} / \mathrm{R}$ stimulation significantly increased the mitochondrial ROS level and JC-1 monomer/aggregate ratio (green/red fluorescence intensity ratio reflects the proportion of mitochondrial depolarization) level (Fig. 9c-d). As shown in Fig. 9e, H/R insult significantly increased autophagosomes and autolysosomes dots than that in LG condition. However, HG decreased the level of autophagic flux by down-regulating the autophagosomes and autolysosomes dots than in LG group. In $\mathrm{HG}+\mathrm{H} / \mathrm{R}$ group, the autophagic flux was increased than that in HG group, but the increased level is obviously decreased compared with $\mathrm{LG}+\mathrm{H} / \mathrm{R}$ group. These results indicate that under normal glucose condition, $\mathrm{H} / \mathrm{R}$ treatment can significantly increase the level of autophagy; while under high glucose condition, the basal level of autophagy is down-regulated, and the increase amplitude of autophagy level after $\mathrm{H} / \mathrm{R}$ insult is significantly reduced.

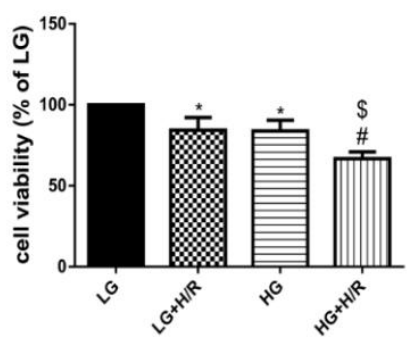

C

Mitochondrial ROS

LG

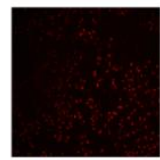

$L G+H / R$

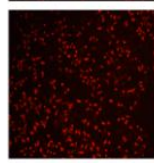

HG
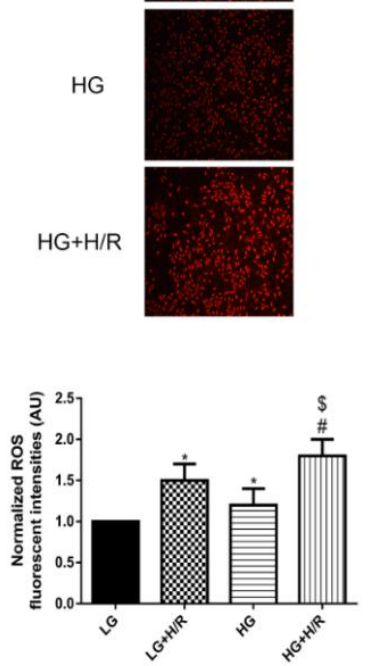

d
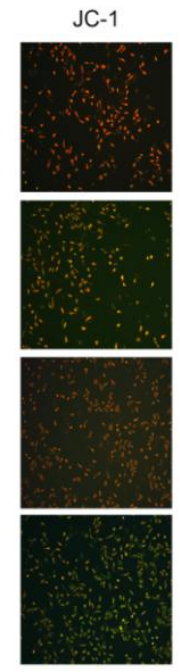

b

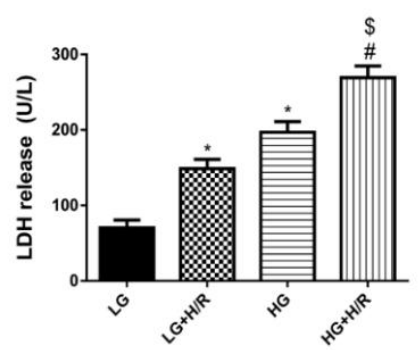

e
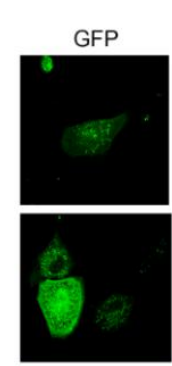

Autophagic flux
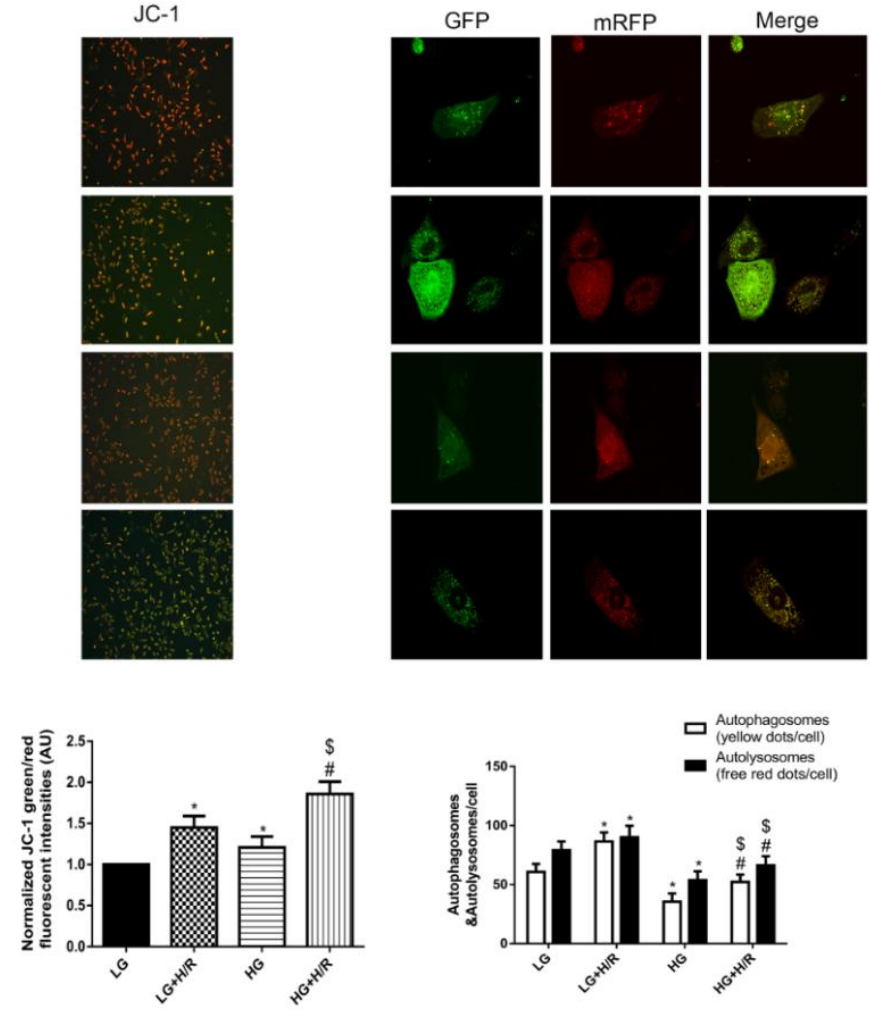
Fig. 9 HG aggravates H/R injury of cardiomyocytes by reducing autophagic flow levels and mitochondrial function. (a) Cell viability was analysed by CCK-8 kit. (b) Serum level of LDH was detected by ELISA kit. (c-d) Mitochondrial ROS (c) and JC-1 (d) were detected to analyse mitochondrial function. Scale bar: $100 \mu \mathrm{m}$. (e) Tandem fluorescent mRFP-GFP-LC3 adenovirus was used to detect the autophagic flux. Scale bar: $20 \mu \mathrm{m}$. n=6 per group. ${ }^{*} P<0.05 \mathrm{LG}$; ${ }^{\#} P<0.05$ versus $\mathrm{HG} ;{ }^{\$} P<0.05$ versus $\mathrm{LG}+\mathrm{H} / \mathrm{R}$. LG, low glucose; $\mathrm{LG}+\mathrm{H} / \mathrm{R}$, low glucose+hypoxia/reoxygenation; HG, high glucose; $\mathrm{HG}+\mathrm{H} / \mathrm{R}$, high glucose+hypoxia/reoxygenation.

\section{Up-regulated expression of HDAC3 mediates Rev-erbo/BMAL1 pathway in high glucose to increase the vulnerability of $\mathbf{H} / \mathbf{R}$ by inhibiting mitophagy in neonatal rat cardiomyocytes}

To evaluate the roles of HDAC3-orchestrated circadian clock gene oscillations and autophagy in cardiomyocytes under $\mathrm{HG}$ and $\mathrm{H} / \mathrm{R}$ insult, we then detected the mRNA and proteins levels of HDAC3, circadian clock gene and autophagy proteins. As shown in Fig. 10, HG could obviously up-regulate the mRNA levels of HDAC3 (Fig. 10a) and Rev-erba (Fig. 10b), and decrease the mRNA level of BMAL1 (Fig. 10c). These changes were further aggravated by H/R. HDAC3 (Fig. 10d) and Rev-erb $\alpha$ (Fig. 10e) mRNA and protein levels were increased in cardiomyocytes in $\mathrm{HG}+\mathrm{H} / \mathrm{R}$ group compared with $\mathrm{LG}+\mathrm{H} / \mathrm{R}$ group with decreased level of BMAL1 (Fig. 10f). However, decreased autophagy activation levels were detected in neonatal rat cardiomyocytes under HG conditions as shown by the decreased LC3II/I level (Fig. 10i) and increased C/EPB (Fig. 10g) and P62 (Fig. 10h) levels. What's more, the $\mathrm{H} / \mathrm{R}$ stimulation could significantly increased autophagy activation to up-regulate autophagy level under LG condition, and decreased levels of autophagy protein in HG condition shown lower increase degree after $\mathrm{H} / \mathrm{R}$ stimulation compared with $\mathrm{LG}+\mathrm{H} / \mathrm{R}$ group (Fig. $10 \mathrm{~g}-\mathrm{i}$ ). All these results demonstrated that high expression of HDAC 3 under HG condition mediated the circadian clock gene to inhibit the activation of autophagy in cultured neonatal rat cardiomyocytes and further aggravated by $\mathrm{H} / \mathrm{R}$ stimulation. 
a

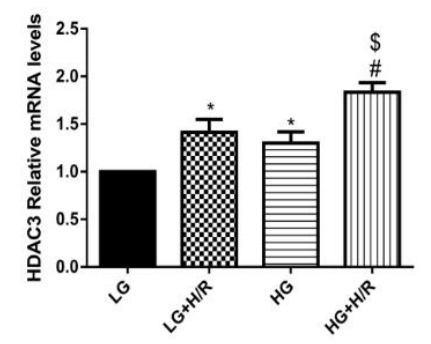

d
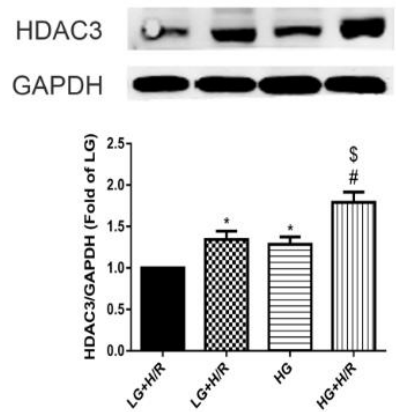

g
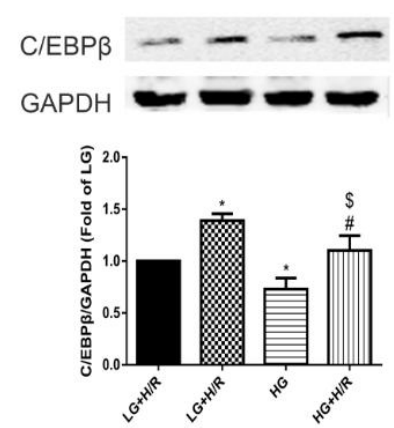

b

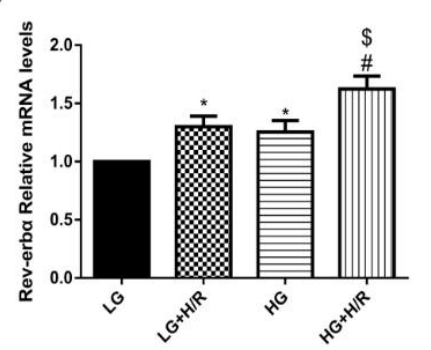

e
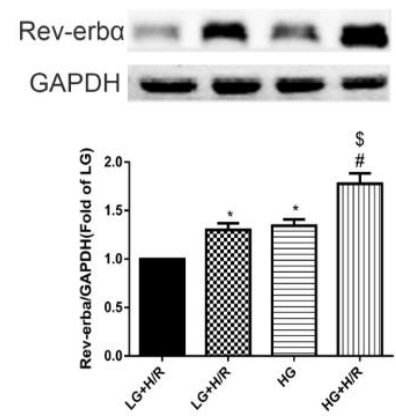

h
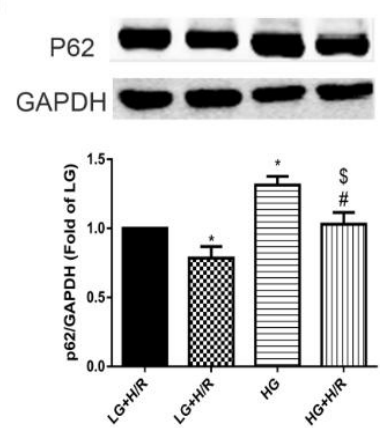

C

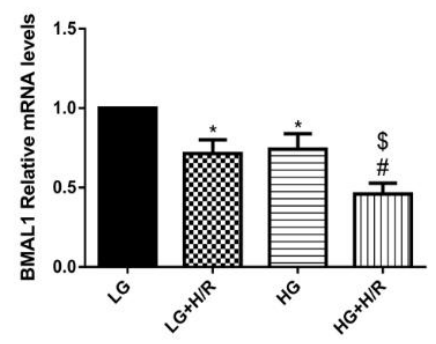

$f$
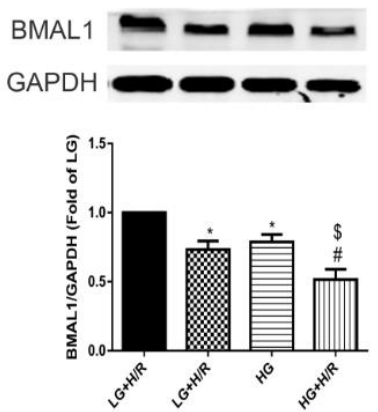

i
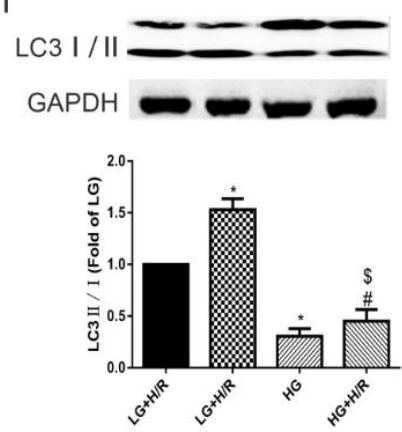

Fig. 10 Increased expression of HDAC3 mediates Rev-erba/BMAL1 in high glucose to increase the vulnerability of H/R by inhibiting mitophagy of neonatal rat cardiomyocytes. (a-c) The mRNA levels of HDAC3 (a), Rev-erb $\alpha$ (b) and BMAL1 (c) were analysed by qPCR. (d-i) The protein levels of HDAC3 (d), Rev-erb $\alpha$ (e), BMAL1 (f), C/EBP $\beta$ (g), P62 (h) and LC3II/I (i) were analysed by western blotting in the cultured neonatal rat cardiomyocytes. $\mathrm{n}=6$ per group. ${ }^{*} P<0.05 \quad$ LG; $\quad{ }^{\#} P<0.05 \quad$ versus $\quad \mathrm{HG} ; \quad{ }^{\$} P<0.05 \quad$ versus $\quad \mathrm{LG}+\mathrm{H} / \mathrm{R}$. LG, low glucose; LG+H/R, low glucose+hypoxia/reoxygenation; $\mathrm{HG}$, high glucose; $\mathrm{HG}+\mathrm{H} / \mathrm{R}$, high glucose+hypoxia/reoxygenation.

\section{Knockdown HDAC3/Rev-erba expression could attenuate $H / R$ injury of neonatal rat cardiomyocytes by regulating BMAL1 to increase mitophagy under HG condition}

To explore the mechanisms of HDAC3 on circadian gene-regulated mitophagy in HG and $\mathrm{H} / \mathrm{R}$-induced injury of neonatal rat cardiomyocytes, we used siRNA to knockdown the expression of HDAC3 or Rev-erb $\alpha$ under HG condition. As shown in Fig. 11a-b, siHDAC3 and siRev obviously decreased $\mathrm{H} / \mathrm{R}$ injury by increasing cell viability and decreasing LDH release in cardiomyocytes with lower mitochondrial ROS (Fig. 11c). As shown in (Fig. 11d), mitochondrial membrane potential was significantly increased by siHDAC 3 and siRev in cultured neonatal cardiomyocytes exposed to $\mathrm{H} / \mathrm{R}$ 
insult under HG condition. As shown in Fig. 11e, the autophagic flux was significantly increased in siHDAC3 and siRev group with increased autophagosomes and autolysosomes dots than in H/R group.

As shown in Fig. 12, the mRNA and protein levels of HDAC3 (Fig. 12a-d) in siHDAC3 group were obviously decreased compared with $\mathrm{H} / \mathrm{R}$ group under $\mathrm{HG}$ condition with the same changes of Rev-erb $\alpha$ mRNA and protein levels (Fig. 12b and e). In addition, compared with H/R group in HG, the mRNA and protein levels of BMAL1 were significant increased (Fig. 12c and f) in H/R+siHDAC3 and $\mathrm{H} / \mathrm{R}+\mathrm{siRev}$ groups. Fig. 12g-i showed that the expression of LC3 II/I was obviously increased after siHDAC3 and siRev infection compared with $\mathrm{H} / \mathrm{R}$ group in $\mathrm{HG}$ condition with decreased $\mathrm{C} / \mathrm{EBP} \beta$ and P62 expression levels.

a

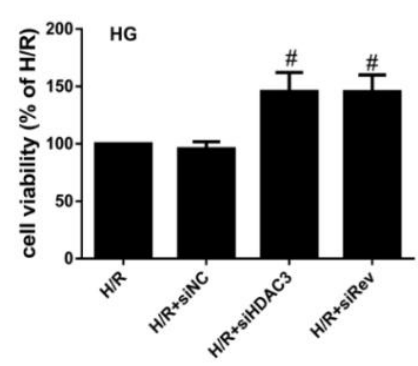

C
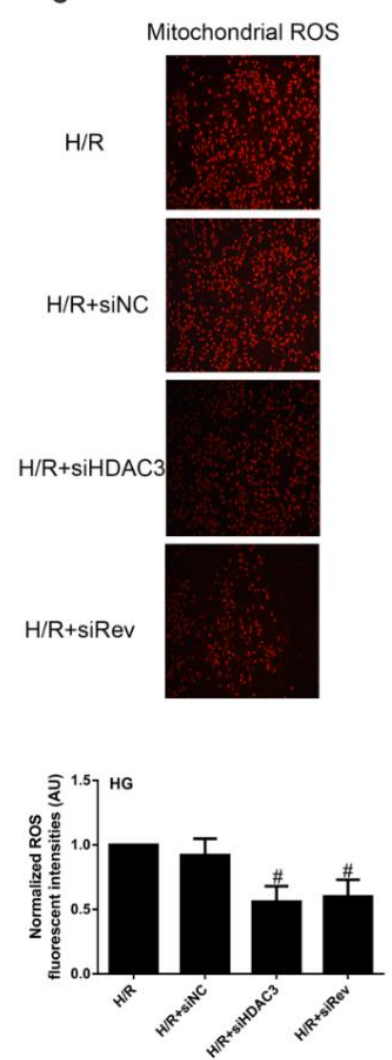

d
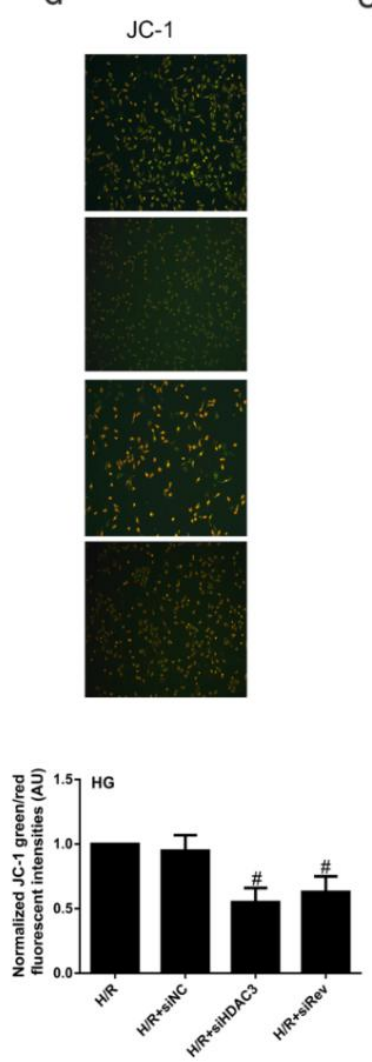

b

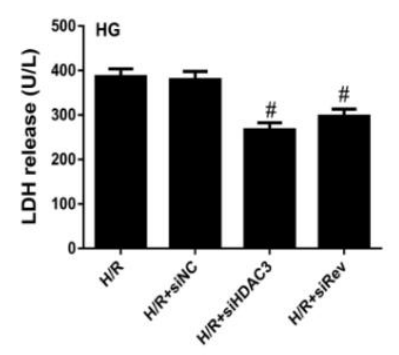

e
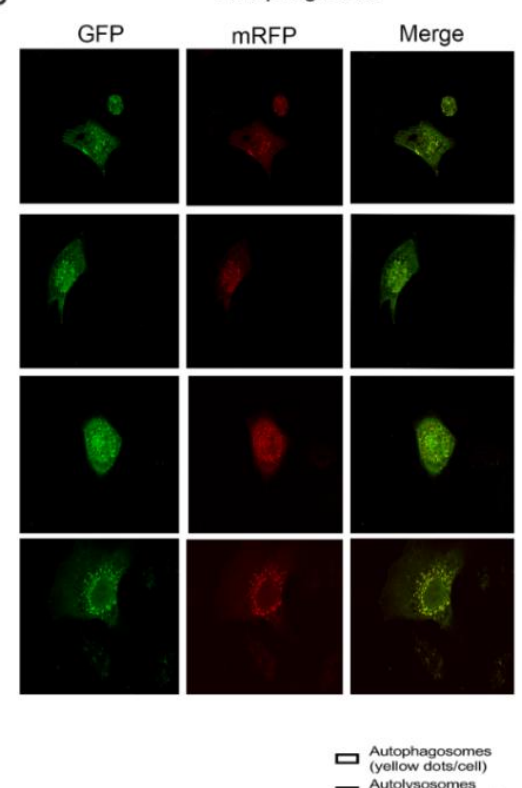

口 $\begin{gathered}\text { Autophagosomes } \\ \text { (yellow dots/cell) } \\ \text { Autolysosomes } \\ \text { (free red dots/cell) }\end{gathered}$

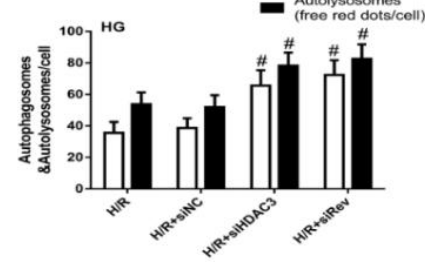

Fig. 11 Knockdown HDAC3/Rev-erb $\alpha$ expression could attenuated H/R injury of neonatal rat cardiomyocytes by increasing autophagic flow levels and mitochondrial function. (a) Cell viability was analysed by CCK-8 kit. (b) The 
serum level of LDH was analysed by ELISA kit. (c-d) Mitochondrial ROS (c) and JC-1 (d) were detected to anlyse mitochondrial function. Scale bar: $100 \mu \mathrm{m}$. (e) Tandem fluorescent mRFP-GFP-LC3 adenovirus was used to detect autophagic flux. Scale bar: $20 \mu \mathrm{m} . \mathrm{n}=6$ per group. ${ }^{\#} P<0.05$ versus $\mathrm{H} / \mathrm{R}$. H/R, hypoxia/reoxygenation; H/R+siNC, hypoxia/reoxygenation+normal control siRNA; H/R+siHDAC3, hypoxia/reoxygenation+ HDAC3 siRNA; H/R+siRev, hypoxia/reoxygenation+Rev-erb $\alpha$ siRNA.

a

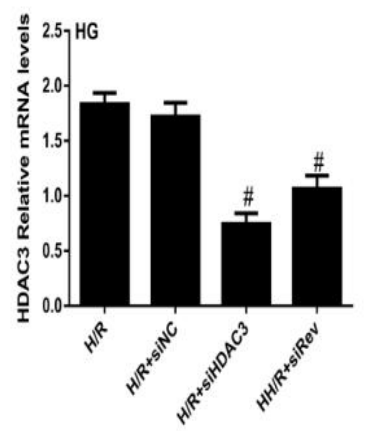

d
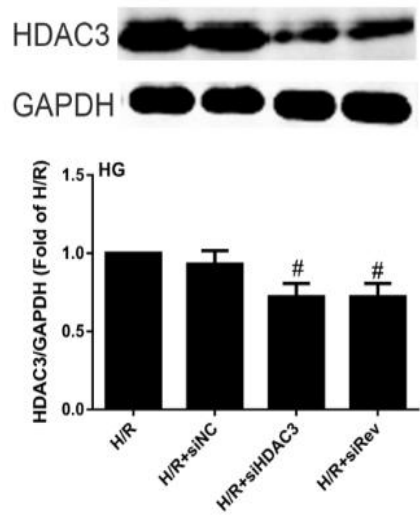

g
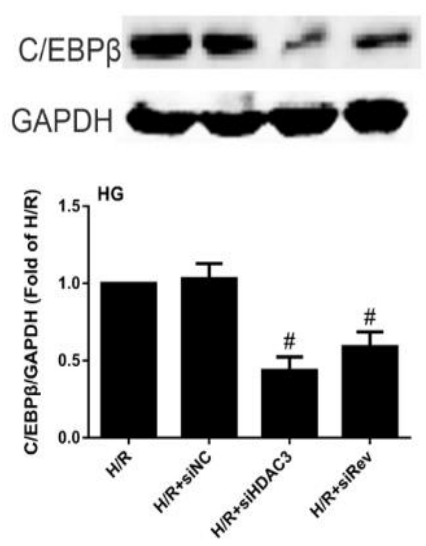

b

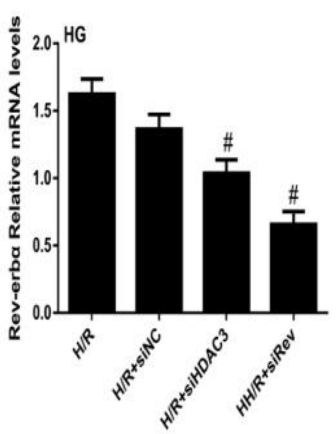

e
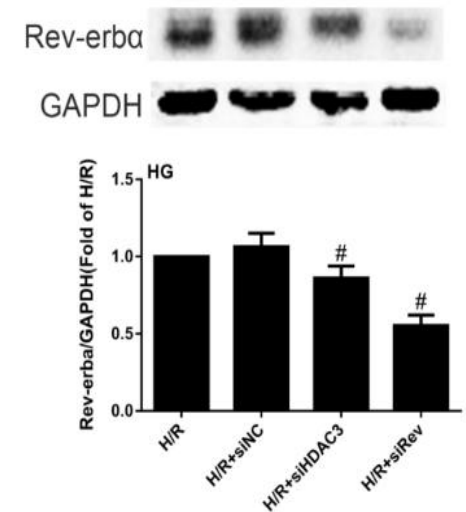

h
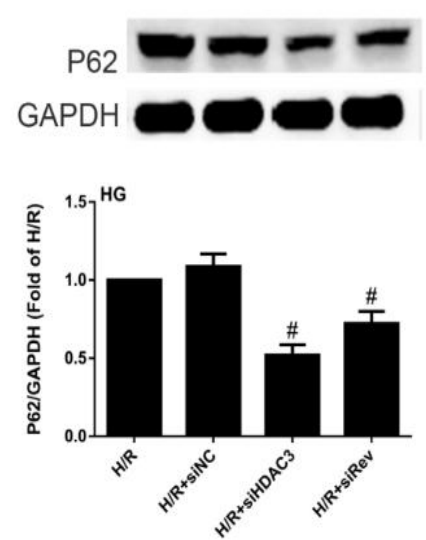

C

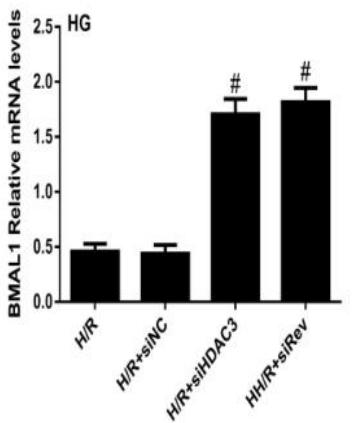

f
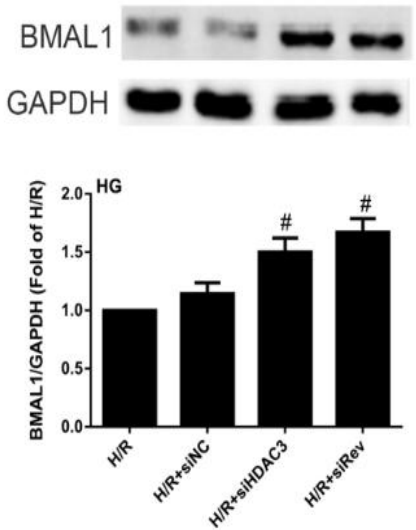

i
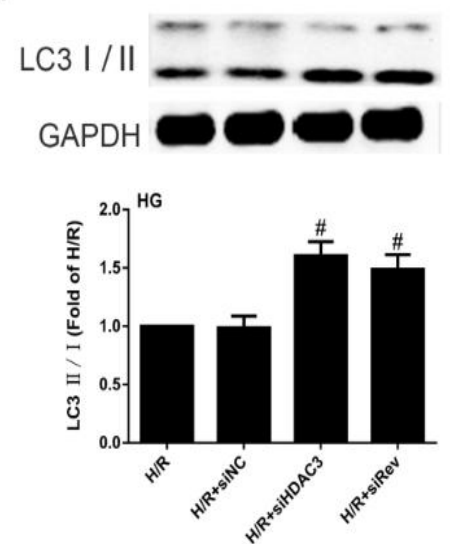

Fig. 12 Knockdown HDAC3/Rev-erb $\alpha$ expression could attenuated $H / R$ injury of neonatal rat cardiomyocytes by up-regulating BMAL1 expression to activate mitophagy of neonatal rat cardiomyocytes under HG condition. (a-c) The mRNA levels of HDAC3 (a), Rev-erba (b) and BMAL1 (c) were analysed by qPCR. (d-i) The protein levels of 
HDAC3 (d), Rev-erba (e), BMAL1 (f), C/EBP 3 (g), P62 (h) and LC3II/I (i) were analysed by western blotting in the cultured neonatal rat cardiomyocytes. $\mathrm{n}=6$ per group. ${ }^{\#} P<0.05$ versus $\mathrm{H} / \mathrm{R}$. H/R, hypoxia/reoxygenation; $\mathrm{H} / \mathrm{R}+\mathrm{siNC}$, hypoxia/reoxygenation+normal control siRNA; H/R+siHDAC3, hypoxia/reoxygenation+ HDAC3 siRNA; H/R+siRev, hypoxia/reoxygenation+Rev-erb $\alpha$ siRNA.

\section{Discussion}

Diabetic patients have higher risk on cardiovascular events compared with non-diabetic patients, and diabetes are more sensitive to ischemic heart diseases. A variety of humans and laboratory animals studies have shown that circadian clocks regulate biological cardiovascular rhythms in both health and disease, which also plays an significant role in myocardial ischemia disease [10-12]. Myocardial mitophagy rhythm disorder is an important pathophysiological feature of MI/RI. Our previous studies have confirmed that mitophagy is impaired during type 1 diabetic MI/RI, and Rap can partially restore the mitophagy level and rhythm of cardiomyocytes, thereby reducing MI/RI [6,20]. In this study, we first explored the roles of HDAC3-orchestrated circadian clock gene oscillations that mediated mitophagy in diabetic MI/RI and neonatal rat cardiomyocytes H/R injury under HG condition. Novel study findings were as follows: First, diurnal variations were initiate in myocardial infarction after $\mathrm{MI} / \mathrm{R}$ in non-diabetes and there is more severity $\mathrm{MI} / \mathrm{RI}$ in diabetes rats without diurnal variations. Second, HDAC3 level was found to be up-regulated in diabetic myocardium and primary cardiomyocytes under HG condition. Third, up-regulated HDAC3 expression in diabetes mediates Rev-erb $\alpha$ BMAL1 pathway to increase the vulnerability of MI/RI. Fourth, HDAC3 mediated circadian gene expression oscillations disrupted to impair mitochondrial function and induce mitophagy dysfunction in diabetic MI/R rats to increase the injury. Finally, cardiac-specific HDAC3 knockdown restored the diabetes-induced vulnerability to MI/RI in diabetes and inhibition of HDAC3 showed beneficial effects against diabetic MI/RI. Conclusively, our present results suggest that HDAC3-orchestrated circadian clock gene oscillations is a novel endogenous protective receptor against diabetic MI/RI.

In recent years, more and more studies have shown that clock gene oscillation plays an significant role in heart disease; the myocardial infarct size and injury caused by $\mathrm{I} / \mathrm{R}$ at different time points during the day are related to circadian clock [11-13]. This study describes a marked circadian distribution of MI/RI in non-diabetic and diabetic rats, depending on the timing of coronary occlusion. Our results indicate that myocardial infarct size and the degree of injury caused by MI/R at different time points in non-diabetic rats are time-dependent and exhibit greatest infarct sizes at ZT12, which is consistent with previous studies [11]. ZT12 corresponds to the sleep-to-wake period of nocturnal rodents. Therefore, the diurnal oscillations and responsiveness in the stimulation (ie., ischemia) are in phase. However, the myocardial infarct size of diabetic rats was significantly increased than non-diabetic rats with more severity myocardial damage, and the myocardial injury at different time points was not significantly fluctuated. These findings are similar to previous reports about circadian cardiovascular effects in humans such as the onset of myocardial infarction or sudden cardiac death [26]. Genetic modulation of circadian clock timing, resulting in subtle circadian dyssychronization accelerated cardiac and renal disease, which is rescued by light/dark cycle-mediated circadian 
resynchronization [27]. These pre-clinical observations [11-12] and our findings may have implications for the development of interventions aimed to reduce the infarct size in clinical trials, they demonstrate that the timing of coronary occlusion will affect the subsequent infarction extent.

Many factors may ultimately affect infarct size following I/R. Clinical and experimental studies have shown that diabetes is more intolerant to MI/RI than non-diabetes and is resistant to various therapeutic methods [28-29]. Hyperglycaemia, hyperlipidaemia, hyperinsulinaemia, and insulin resistance enhanced oxidative stress to activate mitochondrial dysfunction and excessive cytokine generation in the diabetic myocardium so that diabetes cannot be tolerated MI/R. Because glucose and free fatty acid metabolism are regulated by cardiomyocyte circadian clock, the tolerance to ischemia may exhibit variability associated with circadian rhythms [30]. The biological clock is an important endogenous regulation mechanism widely existed in living organisms. The clock gene oscillates and regulates important energy metabolism pathways such as glucose and fat metabolism, so that energy changes exhibit important characteristics-rhythmical to adapt to physiological functions. More and more studies have shown that the disorders and destruction of circadian rhythm play a key role in the development of metabolic diseases such as diabetes, atherosclerotic plaque formation and ischemic heart disease [31]. Study has showed that the loss of synchronization clock with its environment in diabetic heart may effect the development of diabetes [9]. In our study, we found that compared with non-diabetic rats, the circadian rhythm gene oscillations of diabetic rats are attenuated in the heart manifested by the alteration of clock output gene Rev-erb $\alpha$ and BMAL1 rhythmicity in SZT-induced diabetes, which is consistent with the results of Young et al [9]. In further studies, we found that diabetic MI/RI at different time points showed the same attenuation pattern as clock gene rhythm Rev-erb $\alpha$ and BMAL1, showing no significant fluctuations with more serious injury, compared with non-diabetic rats.

As a member of class I HDAC family, HDAC3 is a important factor to regulate glucose and fat metabolism, and also a pivotal element in the circadian rhythm negative feedback loop. HDAC3 null mice prevent glucose tolerance and insulin resistance by lowering blood glucose [32]. Injection of HDAC inhibitor before or during reperfusion can reduce infarct size by $40 \%$ and maintain cardiac systolic function [33]. Rev-erb $\alpha$ is involved in the development of diabetes as an orphan nuclear receptor that regulates sugar and lipid metabolism and energy homeostasis. Rev-erb $\alpha$ promotes transcriptional repression to regulate the circadian rhythm, and constitutes the critical negative electrode of the core circadian clock by directly suppressing the expression of the positive clock component BMAL1. HDAC3 is required for the inhibition of Rev-erb $\alpha$ activity. Rev-erb $\alpha$ directly binds to the BMAL1 gene promoter through two RORE (retinoid-related orphan receptors) binding sites and inhibits its activity [34]. What's more, BMAL1 is an important regulation mechanisms in circadian rhythm of human energy metabolism. BMAL1 knockout mice show circadian rhythm disorder, $\beta$ cell secretion disorder and hyperglycemia, impaired glucose tolerance, and finally development into diabetes [35-36]. In the model of type 1 diabetic rats, the expression of the core clock genes (BMAL1, Per1/2/3) and the output genes (dbp, hlf) showed a significant phase shift, which was manifested by the advancement of the rhythm [32]. These studies suggest that the clock rhythm and stability are important mechanisms for maintaining the body's glucose metabolism balance. Recent studies have indicated that the degree of myocardial injury, cardiac function changes and 
myocardial remodeling after AMI are closely related to the circadian rhythm of the environment in which they are located; the oscillating amplitude of the circadian clock genes (BMAL1, Rev-erba) in the ischemic region exacerbates the development of myocardial infarction and the occurrence of heart failure, suggesting that myocardial damage is related to the circadian rhythm regulated by the circadian clock gene [11,37-38]. Therefore, the HDAC3-orchestrated Rev-erba/BMAL1 gene oscillation participates in the biological clock rhythm and energy metabolism process, and acts an extremely significant role in diabetes and ischemic heart disease. As previous reported, we established type 1 diabetic animal models by intraperitoneal injection STZ. After 8 weeks, the rhythmicity of Rev-erb $\alpha$ and BMAL1 in hearts of diabetic rats was altered and showed an attenuated amplitude compared with that of non-diabetic rats. No significant differences were observed in the zenith (peak) or nadir (trough) of the gene expression rhythms in diabetic rats. We hypothesize that these observations are due to the changes in the circadian rhythms of zeitgebers in diabetes. These results are consistent with a recently published study [32]. After MI/R insult, there was aggravated injury in diabetic rats than in non-diabetes, in consistent with increased expression of HDAC3 and Rev-erb $\alpha$ with lower level of BMAL1 which showed different rhythms compared with control hearts. Further study indicated that inhibition of HDAC3 expression by AAV9-HDAC3 protected against diabetic $\mathrm{MI} / \mathrm{RI}$ by lowering the expression of Rev-erb $\alpha$ to up-reulate the level of BMAL1. These findings emphasized that HDAC3 orchestrated the alterations of circadian clock Rev-erb $\alpha$ and BMAL1 in the heart are probably due to the changes in circulating zeitgebers during diabetes causing a loss of synchronization in stimulus-response coupling, and play a role in aggravated MI/RI of diabetes.

Autophagy is a conservative catabolic process that leads to lysosomal degradation of cellular components. During normal condition, autophagy is involved in tissue and organ remodeling. Under physiological conditions, it is tightly regulated because acts as a housekeeper in the removal of misfolded proteins and damaged organelles. Studies have shown that autophagy activation shows strong circadian rhythms in several tissues including liver, heart, and skeletal muscle [22]. Mitophagy is a key endogenous protection mechanism that maintains the integrity of the entire mitochondrial network, oxidative balance and cell survival. Increased oxidative stress induced by hyperglycemia and hyperlipidemia in diabetic conditions can lead to mitophagy dysfunction [39]. Studies have reported that mitophagy is impaired during $\mathrm{MI} / \mathrm{R}$, and our previous studies have confirmed that mitophagy is more damaged during MI/RI in type 1 diabetes $[6,26]$. Myocardial mitophagy rhythm disorder is an important pathophysiological feature of MI/RI and the autophagy agonist Rap partially restores the mitophagy level and rhythm of cardiomyocytes, thereby reducing the degree of MI/RI [6,40-41]. Here, we found that the level of mitophagy displays robust oscillations and minimums at ZT12 in non-diabetic rat hearts. Moreover, mitophagy level was significantly increased after MI/RI in non-diabetic or diabetic rats. The mitophagy level of diabetic rats was significantly reduced with no rhythmic fluctuation at different times compared with non-diabetic rats. The mitochondrial damage is more serious in diabetic rats after MI/RI, and the increase degree of mitophagy was significantly lower in diabetes than in non-diabetes after MI/RI. These results indicate that mitophagy shows a significant rhythm in non-diabetic rats and activated rhythmically after $I / R$ insult to alleviate myocardial injury. In the type 1 diabetes, the basal level of mitophagy is down-regulated, and 
mitophagy level is insufficient increased after I/R stimulation, thus participating in the diabetic MI/R vulnerability.

As a transcription factor, $\mathrm{C} / \mathrm{EBP} \beta$ is engaged in a variety of physiologic and pathophysiologic processes, especially stress responses. It acts as a new component of the transcriptional network that controls autophagy gene programs and regulates mitophagy activity. Under circadian autophagy rhythm, C/EBP $\beta$ acts a key effect in coordinating the expression of autophagy genes [41]. The level of $\mathrm{C} / \mathrm{EBP} \beta$ is highly rhythmic and mediated by the liver clock in a tissue-autonomous manner [23]. These studies have shown that C/EBP $\beta$ knockout mice exhibit mitophagy rhythm disappearance, while circadian rhythm disorder BMAL1 ${ }^{-/}$mice exhibit a clock-regulated protein $\mathrm{C} / \mathrm{EBP} \beta$ and autophagy-related gene rhythm disorders, thus mitophagy rhythm maintenance depends on the regulation of the core clock gene BMAL1 and is associated with the activation of C/EBP $\beta$ [23-24]. Therefore, the new important feature of mitophagy is rhythm and related to clock gene oscillation. However, the clock gene oscillation loop is through which signaling pathway to regulate the mitophagy rhythm and whether it participates in the MI/RI of diabetes have not been reported so far. In our present study, we found that the expression of $\mathrm{C} / \mathrm{EBP} \beta$ in normal rat myocardial tissue has circadian rhythm while disturbed rhythm in diabetic rats with elevated level. In further study, $\mathrm{C} / \mathrm{EBP} \beta$ expression is up-regulated at a time-dependent manner after MI/RI in non-diabetes rat, and is further up-regulated with oscillation imbalance in diabetic rats after MI/RI. When down-regulated the expression of $\mathrm{HDAC} 3$, the level of $\mathrm{C} / \mathrm{EBP} \beta$ significantly was decreased with attenuated diabetic $\mathrm{MI} / \mathrm{RI}$. These results demonstrated that $\mathrm{C} / \mathrm{EBP} \beta$ as a circadian rhythm gene acts an vital role in diabetic $\mathrm{MI} / \mathrm{RI}$, and the mechnism possibly through regulating mitochondrial function and mitophagy.

There is a link between mitochondrial damage and cell death in cardiomyocytes during hypoxic injury. BNIP3 is a key signaling factor involved in mitochondrial dysfunction, mitophagy and cardiomyocyte death. A unique feature of BNIP3 is its differentiation from other Bcl-2 family members, which can be induced expression during ischemia or hypoxia stress [42]. BNIP3 can trigger mitochondrial disturbances in the mitochondrial inner membrane, leading to respiratory damage, loss of $\Delta \Psi \mathrm{m}$, mitochondrial permeability transition pore opening and cell death. BNIP3 localized in mitochondrie interacts with the autophagosome-localized LC3 acting as a LC3-binding receptor on mitochondrie, and mainly activates excessive mitophagy causing to cell death [43-44]. BNIP3 deficiency significantly reduced neuronal mitophagy and apoptosis, but increases non-selective autophagy following ischemic/hypoxic insult [45]. Previous studies have suggested that BNIP3 as an important element regulates MI/RI and cardiac remodeling [46-48]. In our present study, we found that, the expression of BNIP3 was significantly increased in non-diabetic rats after MI/R stimulated. What's more, the expression of BNIP3 obviously up-regulated in diabetic rats than in non-diabetic rats, and is further up-regulated after MI/RI with aggravated injury. On the other hand, the level of LC3II/I was significantly increased in non-diabetic rats after MI/R stimulated; but the expression of LC3II/I obviously down-regulated in diabetic rats than in non-diabetic rats and is up-regulated after MI/RI with aggravated injury. Compared with non-diabetic rats, the expression of LC3II/I was decreased in diabetic rats after MI/R insult. When down-regulated the expression of HDAC3, the level of BNIP3 significantly decreased with obvious increased LC3II/I expression and mitophagy level to attenuate 
diabetic MI/RI. These results demonstrated that BNIP3 acts an vital role in diabetic MI/RI by inducing mitochondrial dysfunction, mitophagy and cardiomyocyte death in diabetic rats.

\title{
Conclusion
}

our present study demonstrated that HDAC3 is a novel endogenous impact in diabetic MI/RI by regulating circadian genes oscillation then to induce mitophagy dysfunction, and knockdown HDAC3 expression can relieve diabetic MI/RI. Therefore, HDAC3-orchestrated circadian clock gene oscillations may provide a potentially effective molecular goal for the prophylaxis and treatment of diabetic MI/RI.

\begin{abstract}
Abbreviations
MI/RI: myocardial ischemia/reperfusion injury; STZ: streptozocin; LDA: left anterior descending coronary artery; ZT: zeitgeber; C/EBP $\beta$ : CCAAT/enhanced binding protein beta; SD: Sprague-Dawley; AAV9: adeno-associated virus serotype 9; LVDP: left ventricular developed pressure; TTC: 2,3,5-triphenyltetrazolium chloride; N: non-diabetes; D: diabetes; TEM: transmission electron microscopy; MMP: assessment of mitochondrial membrane potential; PVDF: polyvinylidene difluoride; NS: non-diabetes sham; I/R: ischemia/reperfusion; NI/R: non-diabetes ischemia/reperfusion; DS: diabetes sham; DI/R: diabetes ischemia/reperfusion; AAV-HDAC3+I/R: HBAAV9-r-HDAC3 shRNA1-GFP injection+ ischemia/reperfusion; LG: low glucose; H/R: hypoxia/reoxygenation; LG+H/R: low glucose+hypoxia/reoxygenation; HG: high glucose; HG+H/R: high glucose+hypoxia/reoxygenation; H/R+siNC: hypoxia/reoxygenation+normal control siRNA; H/R+siHDAC3: hypoxia/reoxygenation+ HDAC3 siRNA; H/R+siRev: hypoxia/reoxygenation+Rev-erb $\alpha$ siRNA.
\end{abstract}

\section{Conflict of Interests}

The authors declare that there is no conflict of interests regarding the publication of this paper.

\section{Funding}

This study was supported by grants from the National Natural Science Foundation of China (81970722, 81671891 and 81701891).

\section{Authors' contributions}

Z.Q. and Z.Y.X. conceived, designed and supervised the experiments. Z.Q. and H.M. acquired data. conducted data analysis. S.Q.L., B.Z., B.Z., Y.L.Y., and R.X. assisted in the experiment design and performance, discussed and analyzed data and gave conceptual advice. Z.Q. and Z.Y.X. wrote the manuscript. All authors discussed the results and commented on the manuscript.

\section{Acknowledgement}

We would like to thank the Central Laboratory, Renmin Hospital of Wuhan University (Wuhan, Hubei, China) for their support of our study. Pei Zhang and An-Na Du from The Core Facility and Technical Support, Wuhan Institute of Virology, for her help with producing TEM micrographs.

\section{Availability of data and materials}


All data generated or analyzed during this study are included in this published article.

\section{Consent for publication}

Not applicable.

\section{Ethics approval and consent to participate}

All animal procedures conformed to the National Institutes of Health Guide for the Care and Use of Laboratory Animals and were approved by the Laboratory Animal Welfare \& Ethics Committee (IACUC) of Renmin Hospital of Wuhan University.

\section{References}

1. Wang L, Gao P, Zhang M, Huang Z, Zhang D, Deng Q, Li Y, Zhao Z, Qin X, Jin D, Zhou M, Tang X, Hu Y, Wang L. Prevalence and Ethnic Pattern of Diabetes and Prediabetes in China in 2013. JAMA 2017; 317:2515-2523.

2. Packer M. Heart Failure:The Most Important, Preventable, and Treatable Cardiovascular Complication of Type 2 Diabetes. Diabetes Care 2018; 41:11-13.

3. Yahagi K, Kolodgie FD, Lutter C, Mori H, Romero ME, Finn AV, Virmani R. Pathology of Human Coronary and Carotid Artery Atherosclerosis and Vascular Calcification in Diabetes Mellitus. Arterioscler Thromb Vasc Biol 2017; 37:191-204.

4. Qiu Z, Lei S, Zhao B, Wu Y, Su W, Liu M, Meng Q, Zhou B, Leng Y, Xia ZY. NLRP3 Inflammasome Activation-Mediated Pyroptosis Aggravates Myocardial Ischemia/Reperfusion Injury in Diabetic Rats. Oxid Med Cell Longev 2017; 2017:9743280.

5. Leng Y, Wu Y, Lei S, Zhou B, Qiu Z, Wang K, Xia Z. Inhibition of HDAC6 Activity Alleviates Myocardial Ischemia/Reperfusion Injury in Diabetic Rats: Potential Role of Peroxiredoxin 1 Acetylation and Redox Regulation. Oxid Med Cell Longev 2018; 2018:9494052.

6. Zhou B, Lei S, Xue R, Leng Y, Xia Z, Xia ZY. DJ-1 overexpression restores ischaemic post-conditioning-mediated cardioprotection in diabetic rats: role of autophagy. Clin Sci (Lond) 2017; 131:1161-1178.

7. Eckel-Mahan K and Sassone-Corsi P. Metabolism and the circadian clock converge. Physiol Rev 2013; 93:107-135.

8. Hsieh PN, Zhang L, Jain MK. Coordination of cardiac rhythmic output and circadian metabolic regulation in the heart. Cell Mol Life Sci 2018; 75:403-416.

9. Young ME, Wilson CR, Razeghi P, Guthrie PH, Taegtmeyer H. Alterations of the circadian clock in the heart by streptozotocin-induced diabetes. J Mol Cell Cardiol 2002; 34:223-231.

10. Alibhai FJ, Tsimakouridze EV, Reitz CJ, Pyle WG, Martino TA. Consequences of circadian and sleep disturbances for the cardiovascular system. Can J Cardiol 2015; 31:860-872.

11. Montaigne D, Marechal X, Modine T, Coisne A, Mouton S, Fayad G, Ninni S, Klein C, Ortmans S, Seunes C, Potelle C, Berthier A, Gheeraert C, Piveteau C, Deprez R, Eeckhoute J, Duez H, Lacroix D, Deprez B, Jegou B, Koussa M, Edme JL, Lefebvre P, Staels B. Daytime variation of perioperative myocardial injury in cardiac surgery and its prevention by Rev-Erb $\alpha$ antagonism: a single-centre propensity-matched cohort study and a randomised study. Lancet 2018; 391:59-69.

12. Reiter Ronald, Moore Luke, Swingen Cory, Henry Timothy D, Traverse, Jay H. Circadian Dependence of Infarct 
Size and Left-Ventricular Function Following ST-Elevation Myocardial Infarction. Circ Res 2012; 110:105-110.

13. Alenghat T, Meyers K, Mullican SE, Leitner K, Adeniji-Adele A, Avila J, Bućan M, Ahima RS, Kaestner KH, Lazar MA. Nuclear receptor corepressor and histone deacetylase 3 govern circadian metabolicphysiology. Nature 2008; 456: 997-1000.

14. Thiagarajan D, Ananthakrishnan R, Zhang J, O'Shea KM, Quadri N, Li Q, Sas K, Jing X, Rosario R, Pennathur S, Schmidt AM, Ramasamy R. Aldose Reductase Acts as a Selective Derepressor of PPAR $\gamma$ and the Retinoic Acid Receptor. Cell Rep 2016; 15:181-96.

15. Kim YH, Marhon SA, Zhang Y, Steger DJ, Won KJ, Lazar MA. Rev-erba Dynamically Modulates Chromatin Looping to Control Circadian Gene Transcription. Science 2018; 359:1274-1277.

16. Xie M, Kong Y, Tan W, May H, Battiprolu PK, Pedrozo Z, Wang ZV, Morales C, Luo X, Cho G, Jiang N, Jessen ME, Warner JJ, Lavandero S, Gillette TG, Turer AT, Hill JA. Histone deacetylase inhibition blunts ischemia/reperfusion injury by inducing cardiomyocyte autophagy. Circulation 2014; 129:1139-51.

17. Aune SE, Herr DJ, Mani SK, Menick DR. Selective inhibition of class I but not class IIb histone deacetylases exerts cardiac protection from ischemia reperfusion. J Mol Cell Cardiol 2014; 72:138-145.

18. Kung TA, Egbejimi O, Cui J, Ha NP, Durgan DJ, Essop MF, Bray MS, Shaw CA, Hardin PE, Stanley WC, Young ME. Rapid attenuation of circadian clock gene oscillations in the rat heart following ischemia-reperfusion. J Mol Cell Cardiol 2007; 43:744-753.

19. Bulluck H, Nicholas J, Crimi G, White SK, Ludman AJ, Pica S, Raineri C, Cabrera-Fuentes HA, Yellon D, Rodriguez-Palomares J, Garcia-Dorado D, Hausenloy DJ. Circadian variation in acute myocardial infarct size assessed by cardiovascular magnetic resonance in reperfused STEMI patients. Int J Cardiol 2017; 230:149-154.

20. Xue R, Lei S, Xia ZY, Wu Y, Meng Q, Zhan L, Su W, Liu H, Xu J, Liu Z, Zhou B, Xia Z. Selective inhibition of PTEN preserves ischaemic post-conditioning cardioprotection in STZ-induced Type 1 diabetic rats: role of the PI3K/Akt and JAK2/STAT3 pathways. Clin Sci (Lond) 2016; 130:377-392.

21. Dombi E, Mortiboys H, Poulton J. Modulating Mitophagy in Mitochondrial Disease. Curr Med Chem 2018; 25:5597-5612.

22. Ma D, Li S, Molusky MM, Lin JD. Circadian autophagy rhythm: a link between clock and metabolism. Trends Endocrinol Metab 2012; 23:319-325.

23. Ma D and Lin JD. Circadian regulation of autophagy rhythm through transcription factor C/EBPbeta. Autophagy 2012; 8:124-125.

24. Guo L, Huang JX, Liu Y, Li X, Zhou SR, Qian SW, Liu Y, Zhu H, Huang HY, Dang YJ, Tang QQ. Transactivation of Atg4b by C/EBPbeta promotes autophagy to facilitate adipogenesis. Mol Cell Biol 2013; 33:3180-3190.

25. Roul D, Recchia FA. Metabolic alterations induce oxidative stress in diabetic and failing hearts: different pathways, same outcome. Antioxid Redox Signal 2015; 22:1502-14.

26. Muller JE, Ludmer PL, Willich SN, Tofler GH, Aylmer G, Kangos I. Circadian variation in the frequency of sudden cardiac death. Circulation 1987; 75:131-38.

27. Martino TA, Oudit GY, Herzenberg AM, Tata N, Koletar MM, Kabir GM, Belsham DD, Backx PH, Ralph MR, Sole MJ. Circadian rhythm disorganization produces profound cardiovascular and renal disease in hamsters. Am J Physiol Regul Integr Comp Physiol 2008; 294:R1675-1683.

28. Li H, Liu Z, Wang J, Wong GT, Cheung CW, Zhang L, Chen C, Xia Z, Irwin MG. Susceptibility to myocardial ischemia reperfusion injury at early stage of type 1 diabetes in rats. Cardiovascular Diabetology 2013; 12:133-143.

29. Su W, Zhang Y, Zhang Q, Xu J, Zhan L, Zhu Q, Lian Q, Liu H, Xia ZY, Xia Z, Lei S. N-acetylcysteine 
attenuates myocardial dysfunction and postischemic injury by restoring caveolin-3/eNOS signaling in diabetic rats. Cardiovasc Diabetol 2016; 15:146.

30. Durgan DJ, Pulinilkunnil T, Villegas-Montoya C, Garvey ME,Frangogiannis NG, Michael LH, Chow C-W, Dyck JRB, Young ME. Ischemia/reperfusion tolerance is time-of-day-dependent. mediation by the cardiomyocyte circadian clock. Circ Res 2010; 106:546-550.

31. Corella D, Asensio EM, Coltell O, Sorlí JV, Estruch R, Martínez-González MÁ, Salas-Salvadó J, Castañer O, Arós F, Lapetra J, Serra-Majem L, Gómez-Gracia E, Ortega-Azorín C, Fiol M, Espino JD, Díaz-López A, Fitó M, Ros E, Ordovás JM. CLOCK gene variation is associated with incidence of type-2 diabetes and cardiovascular diseases in type-2 diabetic subjects: dietary modulation in the PREDIMED randomized trial. Cardiovasc Diabetol 2016; $15: 4$.

32. Sun Z, Singh N, Mullican SE, Everett LJ, Li L, Yuan L, Liu X, Epstein JA, Lazar MA. Diet-induced lethality due to deletion of the Hdac3 gene in heart and skeletal muscle. J Biol Chem 2011; 286:33301-9.

33. Sharifi-Sanjani M, Shoushtari AH, Quiroz M, Baust J, Sestito SF, Mosher M, Ross M, McTiernan CF, St Croix CM, Bilonick RA, Champion HC, Isenberg JS. Cardiac CD47 drives left ventricular heart failure through Ca2+-CaMKII-regulated induction of HDAC3. J Am Heart Assoc 2014; 3:e000670.

34. Ikeda R, Tsuchiya Y, Koike N, Umemura Y, Inokawa H, Ono R, Inoue M, Sasawaki Y, Grieten T, Okubo N, Ikoma $\mathrm{K}$, Fujiwara $\mathrm{H}$, Kubo T, Yagita K. REV-ERB $\alpha$ and REV-ERB $\beta$ function as key factors regulating Mammalian Circadian Output. Sci Rep 2019; 9:10171.

35. Marcheva B, Ramsey KM, Buhr ED, Kobayashi Y, Su H, Ko CH, Ivanova G, Omura C, Mo S, Vitaterna MH, Lopez JP, Philipson LH, Bradfield CA, Crosby SD, JeBailey L, Wang X, Takahashi JS, Bass J. Disruption of the clock components CLOCK and BMAL1 leads to hypoinsulinaemia and diabetes. Nature 2010; 466:627-631.

36. Eckel-Mahan K and Sassone-Corsi P. Metabolism and the circadian clock converge. Physiol Rev 2013; 93:107-135.

37. Reiter R, Swingen C, Moore L, Henry TD, Traverse JH. Circadian dependence of infarct size and left ventricular function after ST elevation myocardial infarction. Circ Res 2012; 110:105-110.

38. Bray MS, Shaw CA, Moore MW, Garcia RA, Zanquetta MM, Durgan DJ, Jeong WJ, Tsai JY, Bugger H, Zhang D, Rohrwasser A, Rennison JH, Dyck JR, Litwin SE, Hardin PE, Chow CW, Chandler MP, Abel ED, Young ME. Disruption of the circadian clock within the cardiomyocyte influences myocardial contractile function, metabolism, and gene expression. Am J Physiol Heart Circ Physiol 2008; 294:H1036-H1047.

39. Lorenzo O, Ramirez E, Picatoste B, Egido J, Tunon J. Alteration of energy substrates and ROS production in diabetic cardiomyopathy. Mediators Inflamm 2013; 2013:461967.

40. Ham PB 3rd, Raju R. Mitochondrial function in hypoxic ischemic injury and influence of aging. Prog Neurobiol 2017; 157:92-116.

41. Przyklenk K, Dong Y, Undyala VV, Whittaker P. Autophagy as a therapeutic target for ischaemia /reperfusion injury? Concepts, controversies, and challenges. Cardiovasc Res 2012; 94:197-205.

42. Rabinovich-Nikitin I, Lieberman B, Martino TA, Kirshenbaum LA. Circadian-Regulated Cell Death in Cardiovascular Diseases. Circulation 2019; 139:965-980.

43. Shi RY, Zhu SH, Li V, Gibson SB, Xu XS, Kong JM. BNIP3 interacting with LC3 triggers excessive mitophagy in delayed neuronal death in stroke. CNS Neurosci Ther 2014; 20:1045-55.

44. Wang EY, Gang H, Aviv Y, Dhingra R, Margulets V, Kirshenbaum LA. p53 mediates autophagy and cell death by a mechanism contingent on Bnip3. Hypertension 2013; 62:70-77. 
45. Ma X, Godar RJ, Liu H, Diwan A. Enhancing lysosome biogenesis attenuates BNIP3-induced cardiomyocyte death. Autophagy 2012; 8:297-309.

46. Diwan A, Krenz M, Syed FM, Wansapura J, Ren X, Koesters AG, Li H, Kirshenbaum LA, Hahn HS, Robbins J, Jones WK, Dorn GW. Inhibition of ischemic cardiomyocyte apoptosis through targeted ablation of Bnip3 restrains postinfarction remodeling in mice. J Clin Invest 2007; 117:2825-2833.

47. Hamacher-Brady A, Brady NR, Logue SE, Sayen MR, Jinno M, Kirshenbaum LA, Gottlieb RA, Gustafsson AB. Response to myocardial ischemia/reperfusion injury involves Bnip3 and autophagy. Cell Death Differ 2007; 4:146-57.

48. Hamacher-Brady A, Brady NR, Gottlieb RA, Gustafsson AB. Autophagy as a protective response to Bnip3-mediated apoptotic signaling in the heart. Autophagy 2006; 2:307-309. 


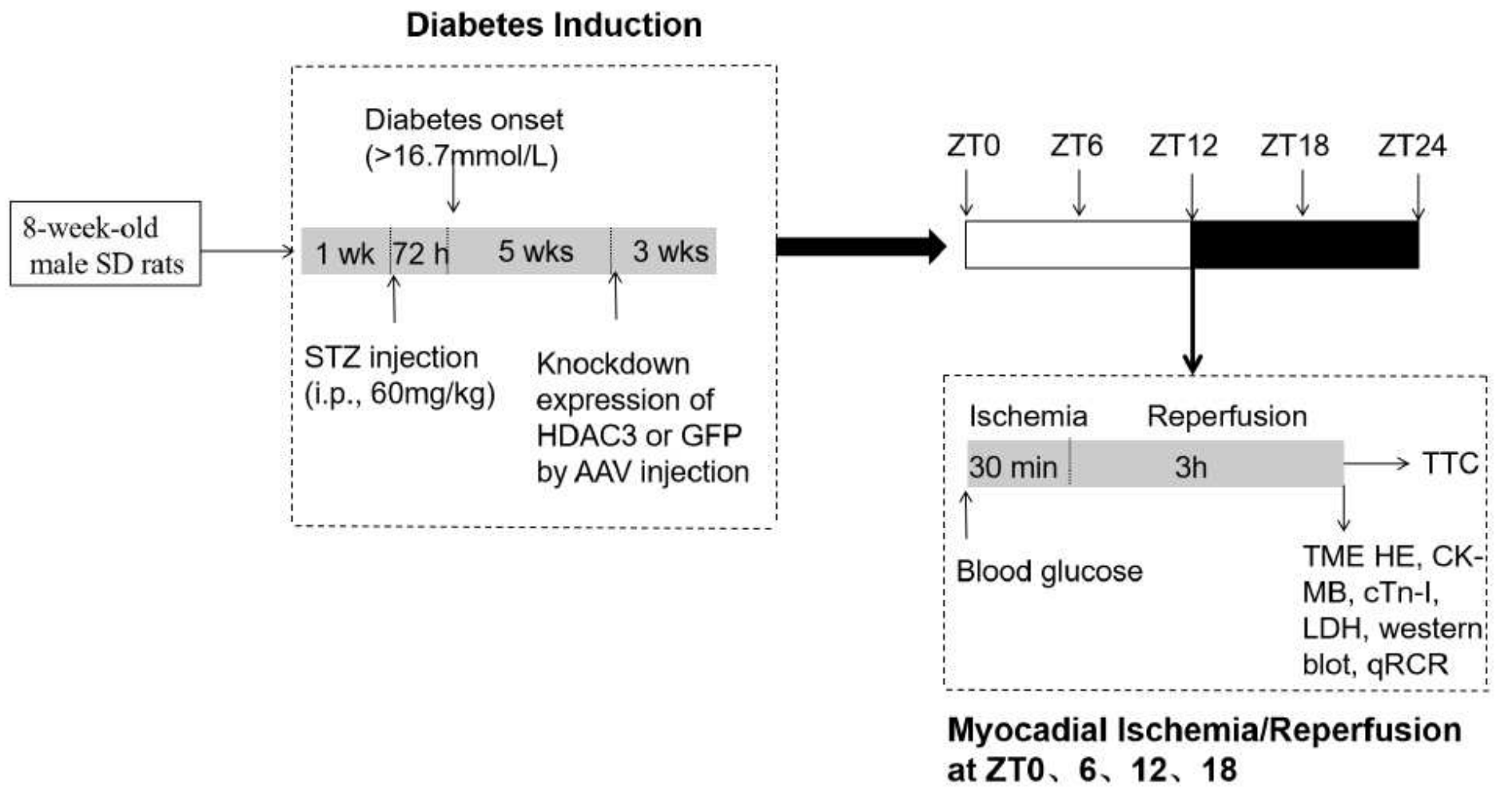

Figure 1

Schematic diagram of experimental program. It illustrates the period of diabetic induction, the time points of adeno-associated virus injection, ischemia/reperfusion administration and data acquisition.
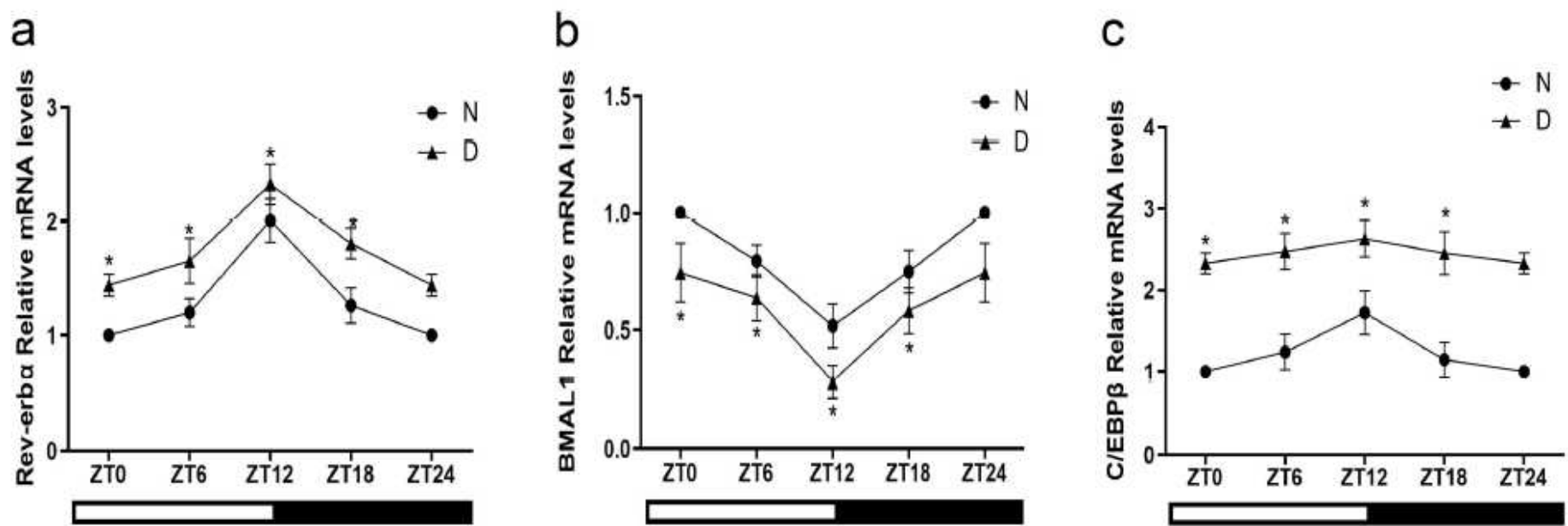

Figure 2

Rev-erba, BMAL1 and C/EBP $\beta$ mRNA diurnal variations in non-diabetic rats (NS) and diabetic rats (DM). $(a-c)$ Expression levels of Rev-erba (a) , BMAL1 (b) and C/EBPB (c) were measured by qPCR over time 
after reperfusion. $\mathrm{n}=6$ per group. ${ }^{*} \mathrm{P}<0.05$ versus $\mathrm{N}$ within $\mathrm{ZT}$. $\mathrm{N}$, non-diabetes; $\mathrm{D}$, diabetes.
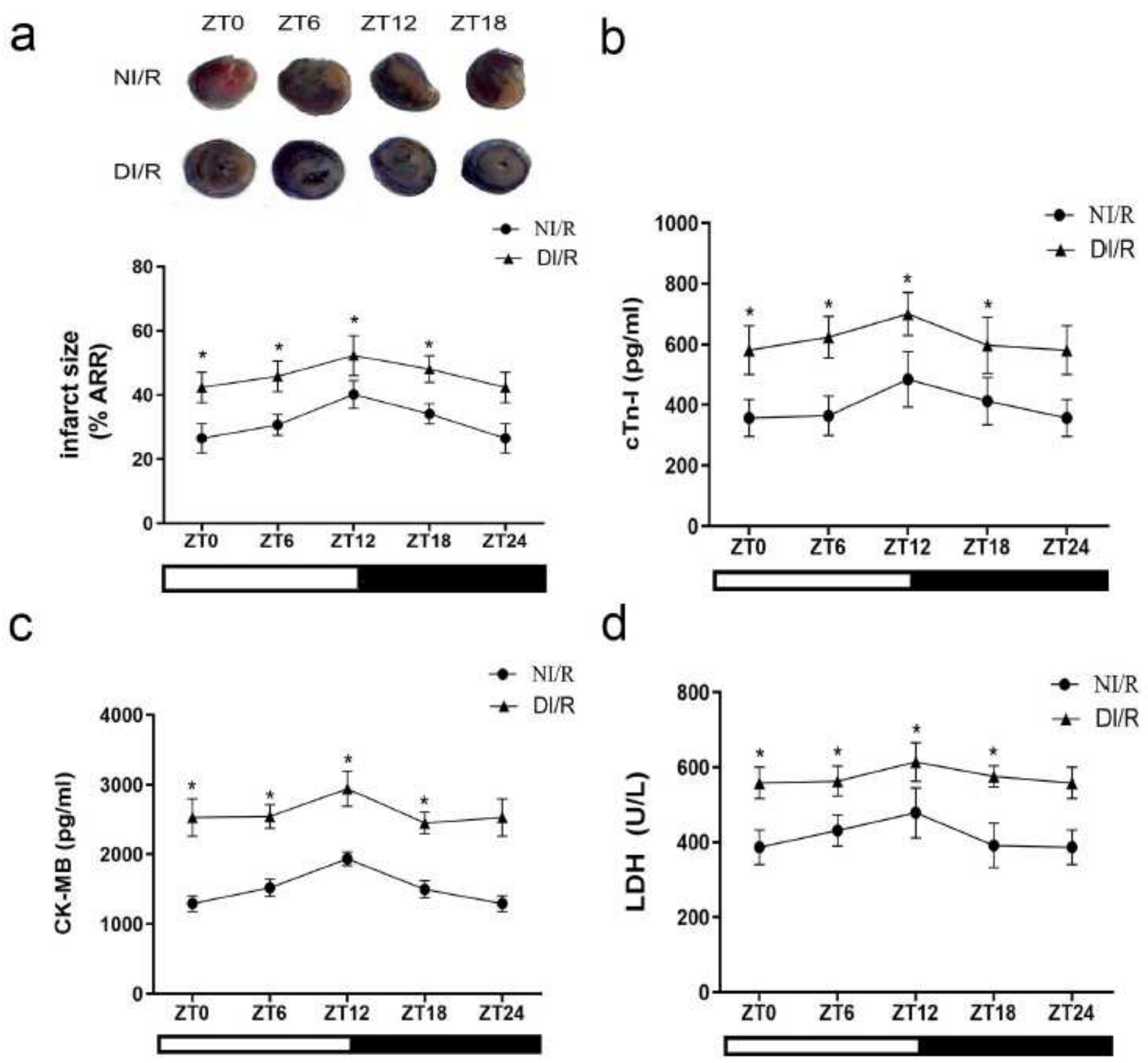

Figure 3

Infarct size, levels of CTn-I, CK-MB and LDH diurnal variations are dependent on time-of day of MI/RI in diabetic and non-diabetic rats. (a) Infarct size was detected by TTC. (b-d) The levels of cTn-I (b), CK-MB (c) and LDH (d) was detected by ELSAL in the surum of non-diabetes and diabetes with or without I/R insult. Scale bar: $2 \mathrm{~mm}$. $\mathrm{n}=6$ per group. ${ }^{*} \mathrm{P}<0.05$ versus NI/R within $\mathrm{ZT}$. NI/R, non-diabetes ischemia/reperfusion; DI/R, diabetes ischemia/reperfusion. 
a
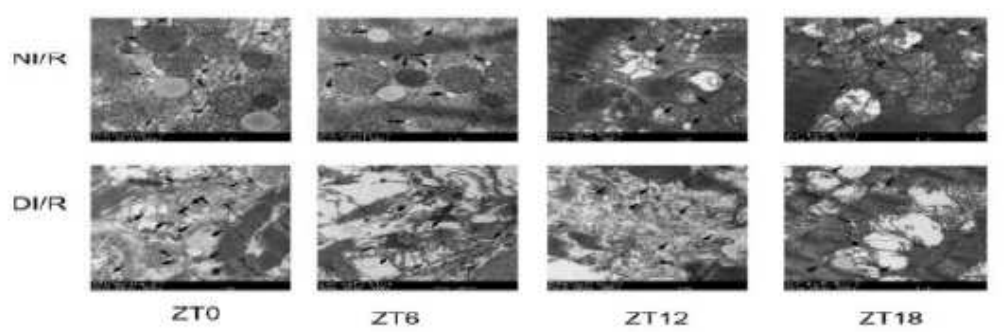

b

C

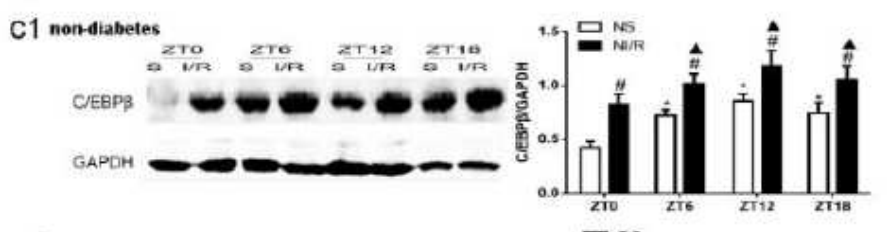

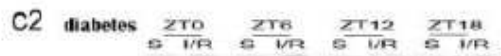
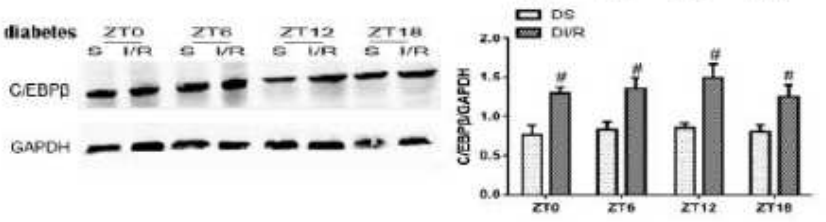

e
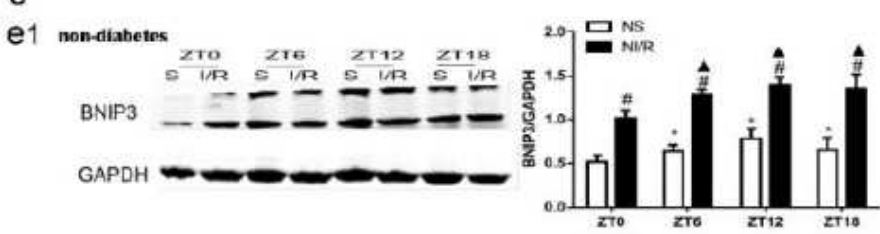

e2
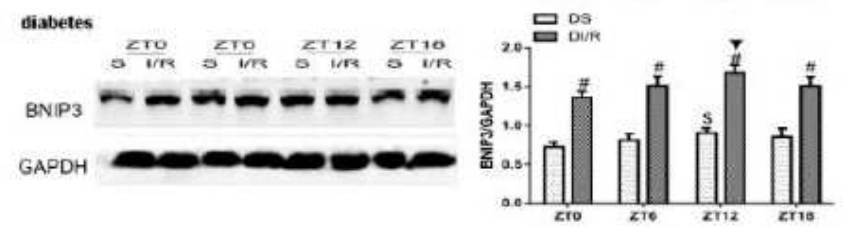

d

d1 non-fiabetes

d2 diatetes

f

f2
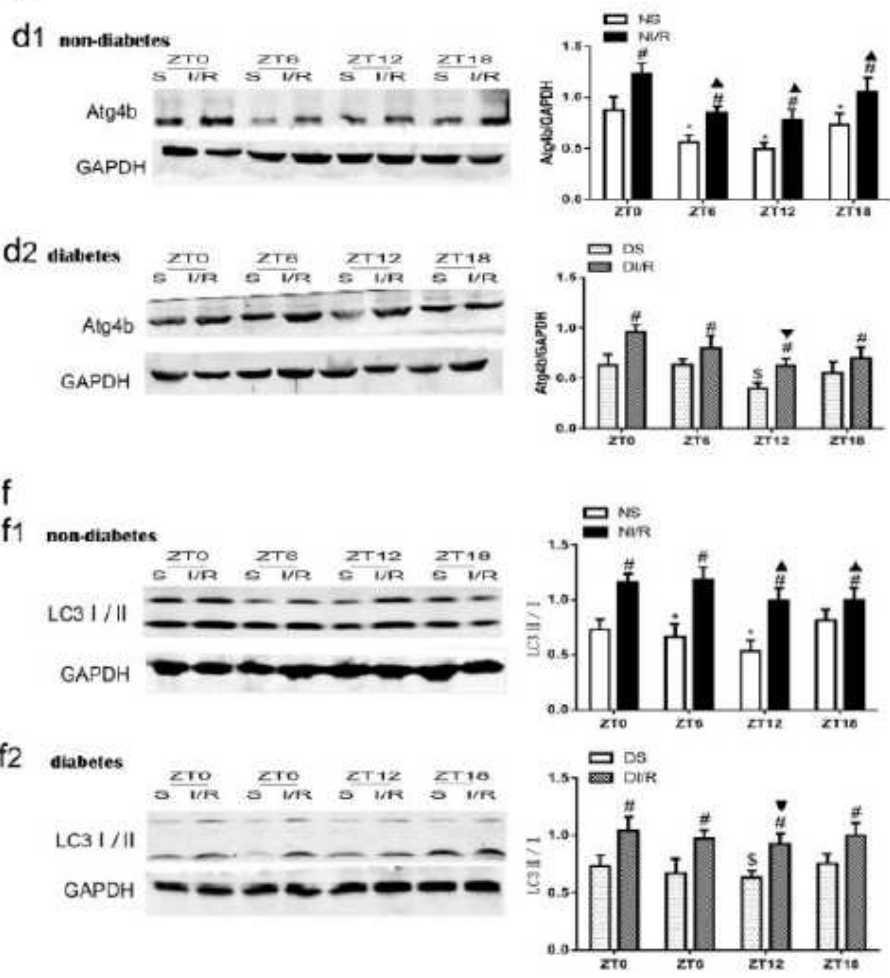

\section{Figure 4}

Mitochondrial damage, autophagosome, and the proteins expression of mitophagy-related genes diurnal variations are dependent on time-of day of $\mathrm{MI} / \mathrm{RI}$ in non-diabetic and diabetic rats. (a-b) The ultrastructural changes and autophagic vacuoles of rat hearts were detected by TEM, Scale bar: $1 \mu \mathrm{m}$. (cf) The protein levels of C/EBP $\beta$ (c), Atg4b (d), BNIP3(e) and LC3区/囚 (f) was detected by western blotting in the myocardial tissues of non-diabetes and diabetes with or without I/R insult. $n=6$ per group. $\S \mathrm{P}<0.05$ versus $\mathrm{NI} / \mathrm{R}$ within $\mathrm{ZT} ; \& \mathrm{P}<0.05$ versus $\mathrm{ZTO}$ within $\mathrm{NI} / \mathrm{R} ;{ }^{*} \mathrm{P}<0.05$ versus $\mathrm{ZTO}$ within $\mathrm{NS} ; \# \mathrm{P}<0.05$ versus sham within $Z T ; \$ P<0.05$ versus $Z T 0$ within $D S ; \otimes P<0.05$ versus $Z T 0$ within $N I / R$; $\otimes P<0.05$ versus $Z T 0$ within DI/R. NS, non-diabetes sham; NI/R, non-diabetes ischemia/reperfusion; DS, diabetes sham; DI/R, diabetes ischemia/reperfusion. 
a

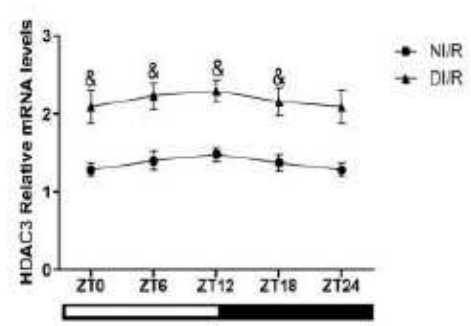

d

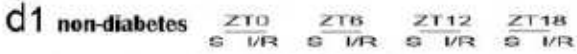

HDAC3

GAPDH

d2
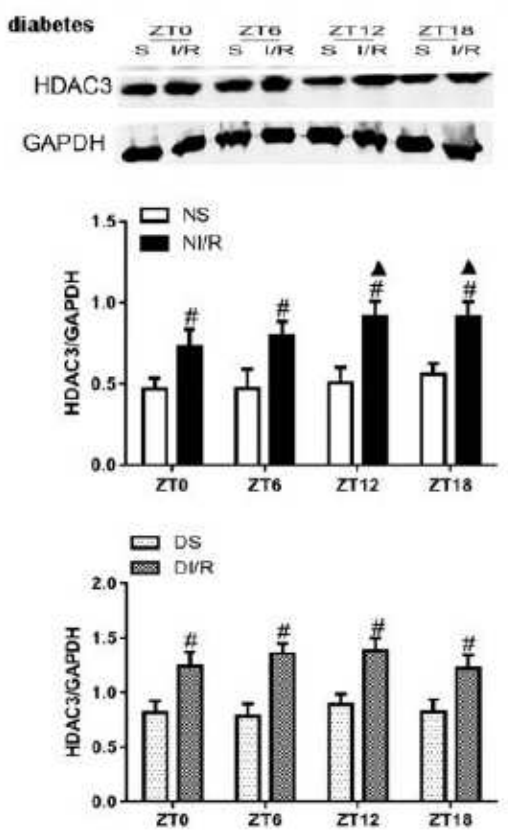

b

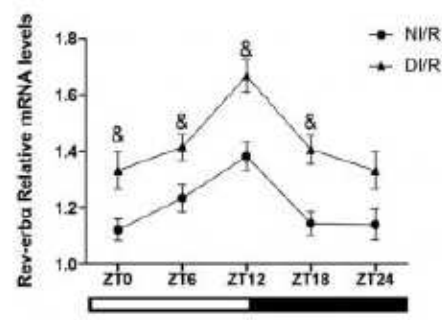

e

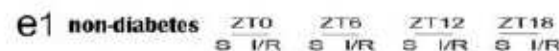

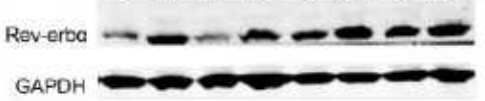

e2
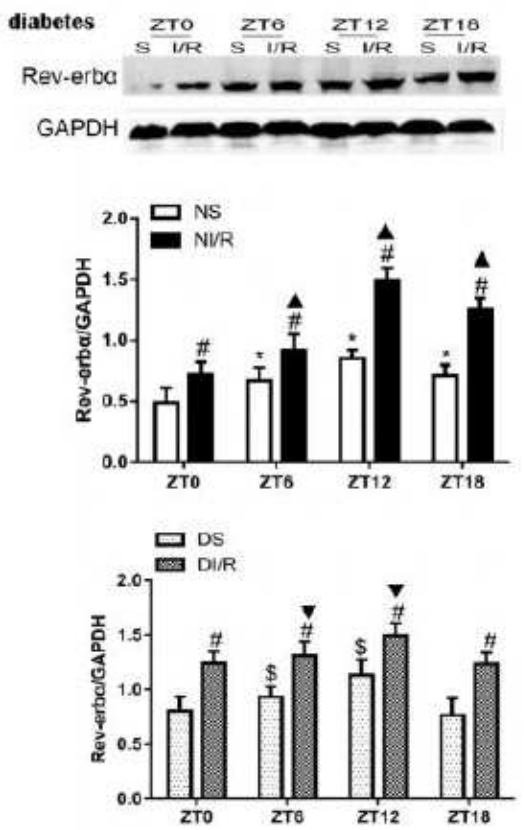

C

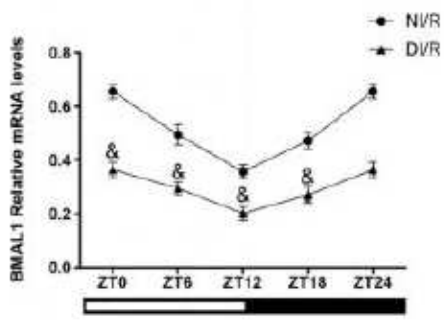

f

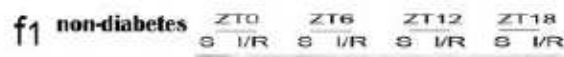
BMAL1 $0-\infty-\infty$ GAPDH

f2
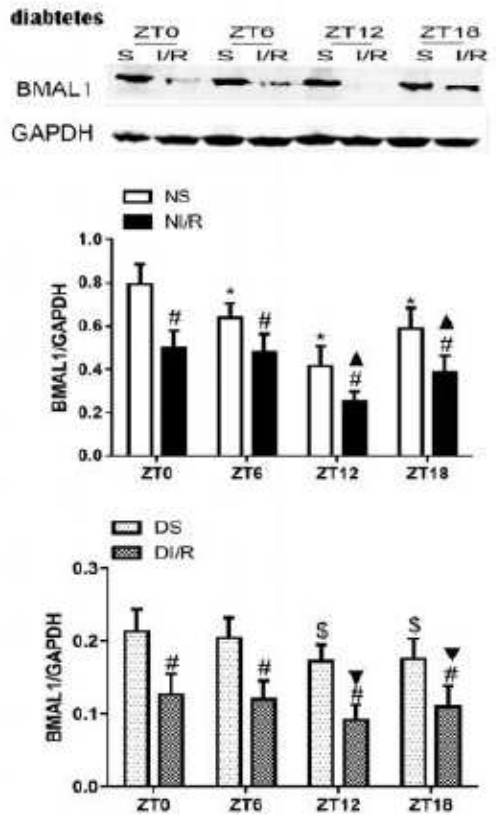

Figure 5

The mRNA and protein expression of HDAC3 and circadian genes (Rev-erba and BMAL1) are dependent on time-of day of MI/RI in non-diabetes and diabetes. (a-c) The mRNA levels of HDAC3 (a), Rev-erba (b) and BMAL1 (c) were analysed by qPCR. (d-f) The protein levels of HDAC3 (d), Rev-erba (e) and BMAL1 (f) was analysed by western blotting in myocadial tissues of non-diabetes and diabetes with or without $\mathrm{MI} / \mathrm{R}$ insult. $n=6$ per group. $\& P<0.05$ versus $N I / R$ within $Z T ;{ }^{*} P<0.05$ versus $Z T 0$ within $N S$; $\# P<0.05$ versus sham within $Z T ; \$ P<0.05$ versus $Z T 0$ within $D S ; \otimes P<0.05$ versus $Z T 0$ within $N I / R ; \otimes P<0.05$ versus $Z T 0$ within DI/R. NS, non-diabetes sham; NI/R, non-diabetes ischemia/reperfusion; DS, diabetes sham; DI/R, diabetes ischemia/reperfusion. 
a

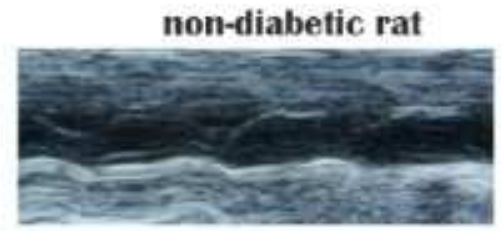

diabetes rat

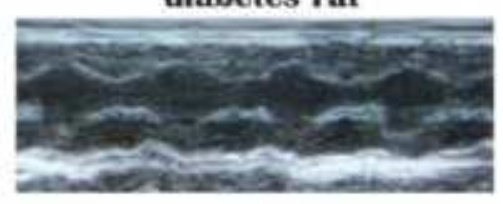

C
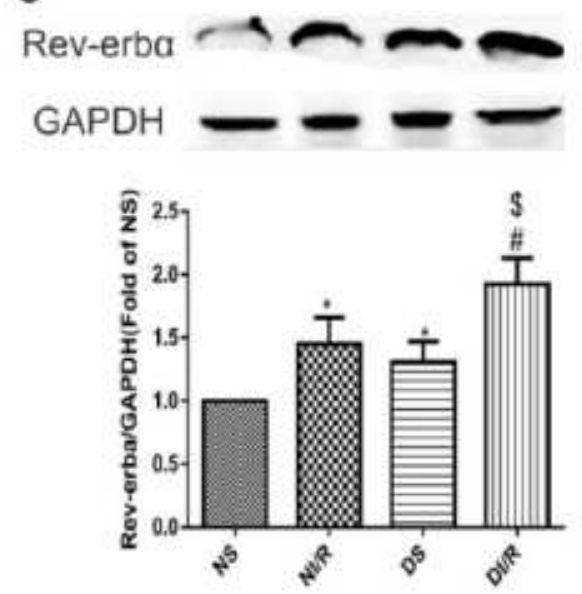

$f$
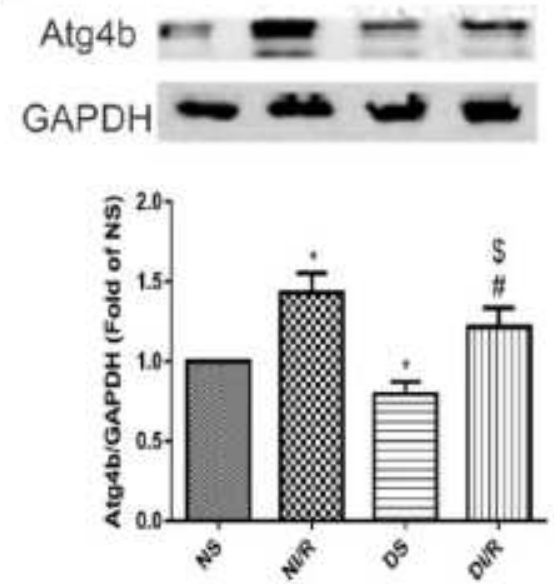

b

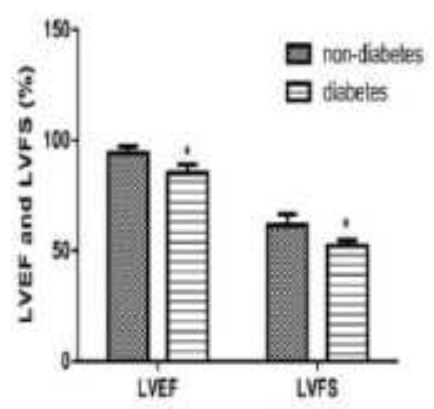

HDAC3

GAPDH

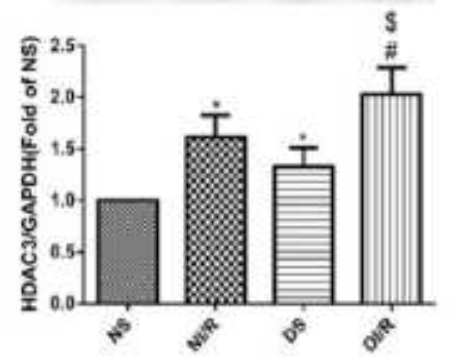

e
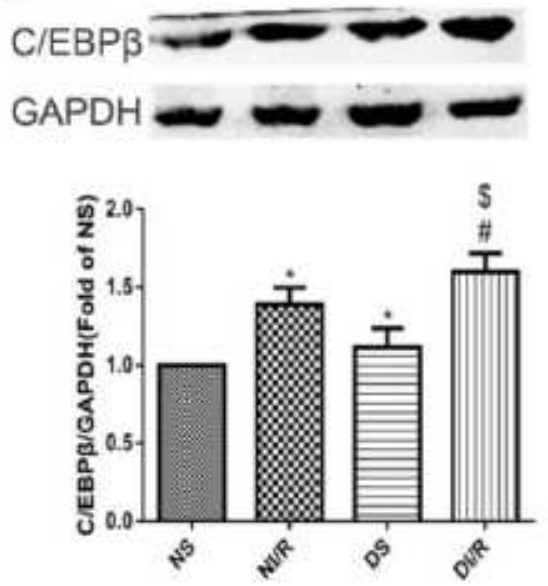

$\mathrm{h}$

g
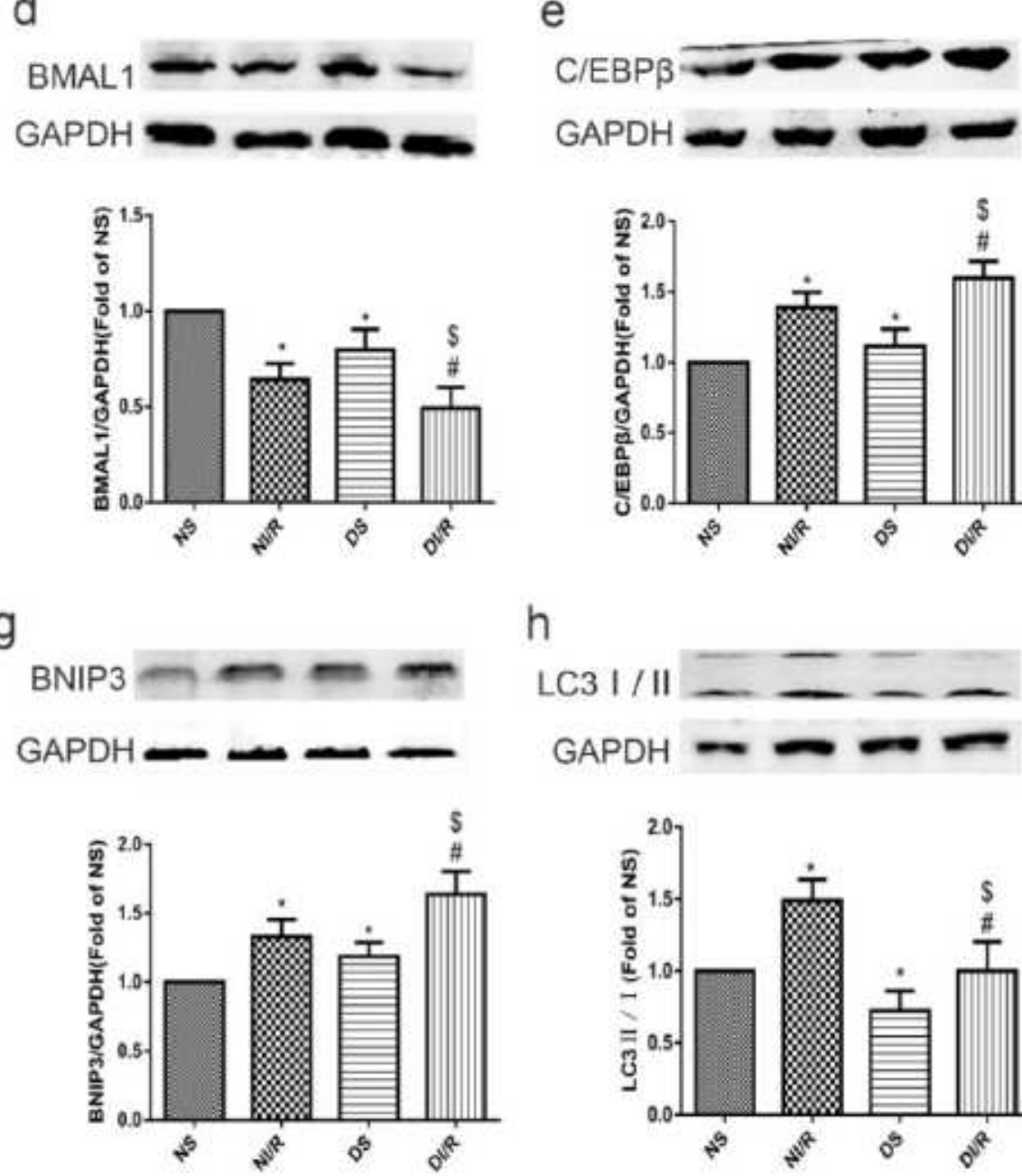

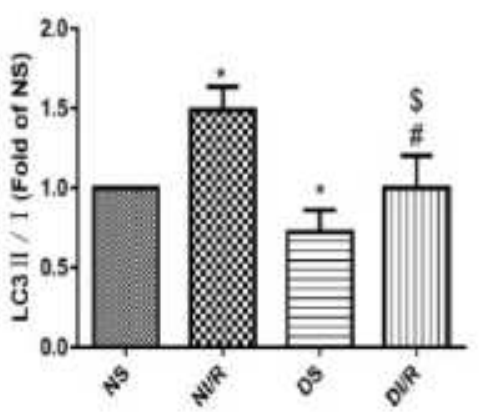

Figure 6

HDAC3 up-regulated expression promotes cardiac dysfunction and down-regulates autophagy by mediated circadian genes in diabetic rats to aggravate MI/RI. (a) Cardiac function was assessed by echocardiography in non-diabetic and diabetic rats. (b-h) The protein levels of HDAC3 (b), Rev-erba (c), BMAL1 (d), C/EBPß (e), Atg4b (f), BNIP3(g) and LC3囚/囚 (h) was detected by western blotting. Scale bar: 2 
mm. $n=6$ per group. ${ }^{*} \mathrm{P}<0.05$ versus $\mathrm{NS} ; \# \mathrm{P}<0.05$ versus $\mathrm{DS} ; \mathrm{SP}<0.05$ versus NI/R. NS, non-diabetes sham; $\mathrm{NI} / \mathrm{R}$, non-diabetes ischemia/reperfusion; DS, diabetes sham; DI/R, diabetes ischemia/reperfusion.

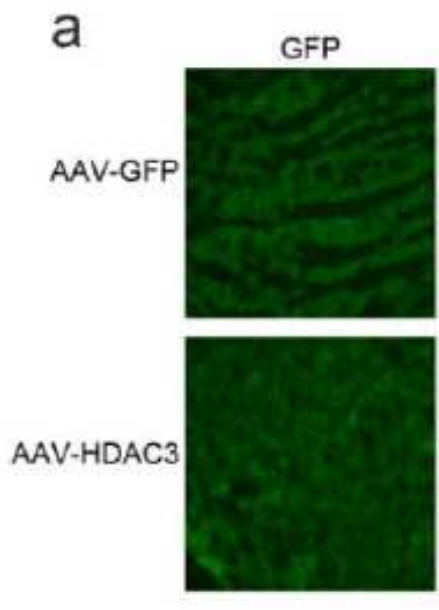

d

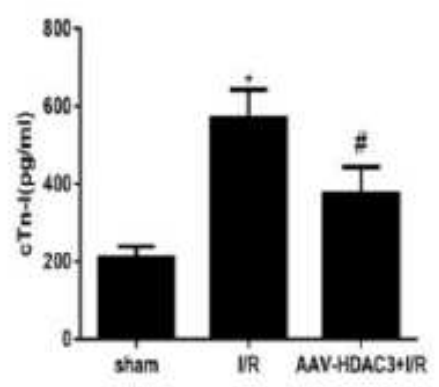

b
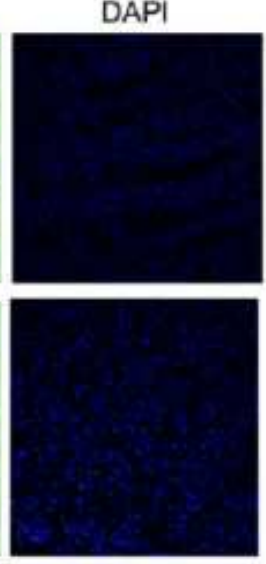

e

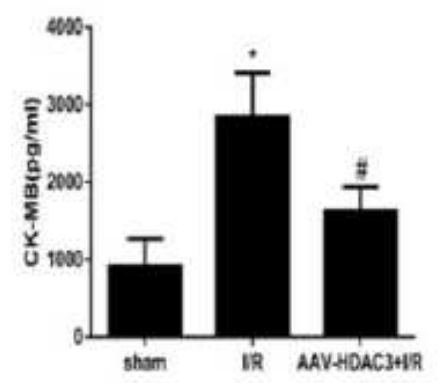

C
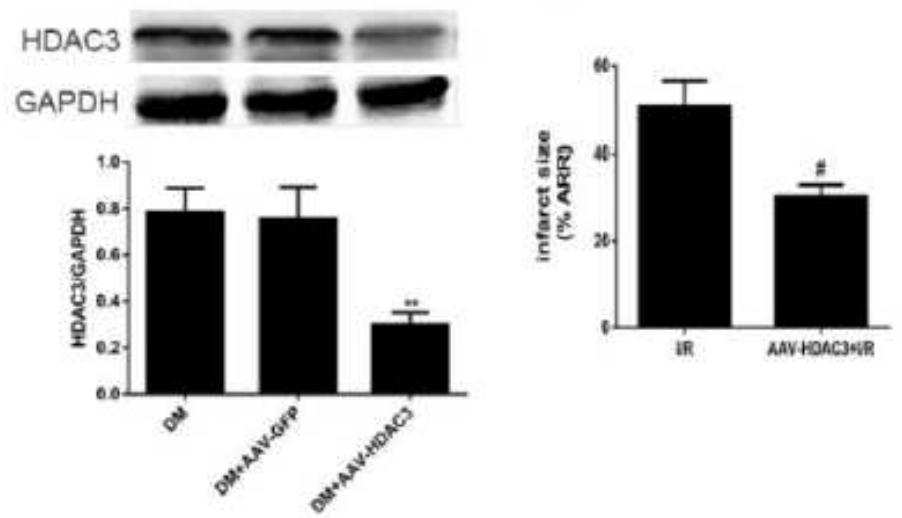

f

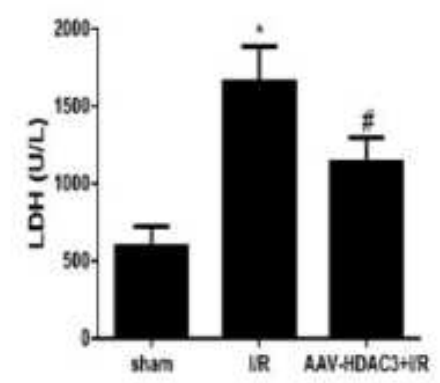

\section{Figure 7}

Knockdown expression of HDAC3 attenuated MI/RI of diabetes. (a) Representative images of immunofluorescence staining for GFP and DAPI in myocardial tissue after AAV-GFP and AAV-HDAC3 transfection. (b) Protein expression of HDAC3 was analyed by western blotting. (c) Infarct size was measured by TTC. (d-f) The serum levels of cTn-I (d), CK-MB (e) and LDH (f) were measured by ELISA assay kits. scale bar $=100 \mu \mathrm{m} . \mathrm{n}=6$ per group. ${ }^{* *} \mathrm{P}<0.01$ versus $\mathrm{DM}$; ${ }^{*} \mathrm{P}<0.05$ versus sham; \#P<0.05 versus I/R. I/R, ischemia/reperfusion; AAV-HDAC3+I/R, HBAAV9-r-HDAC3 shRNA1-GFP injection+ ischemia/reperfusion. 
a

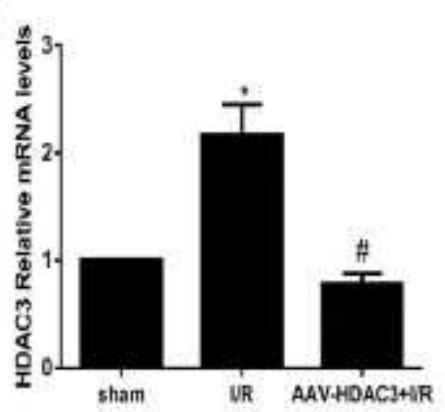

d
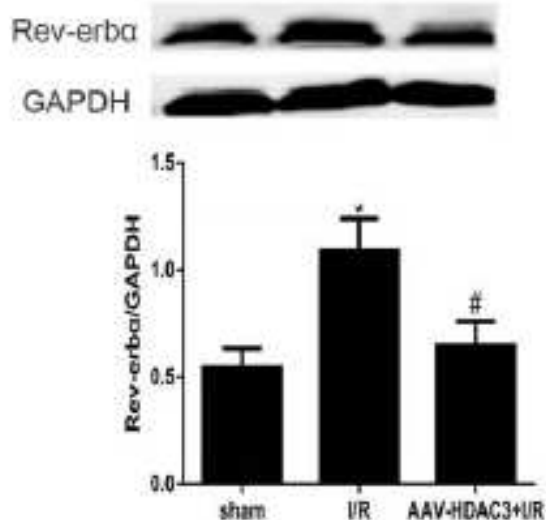

g
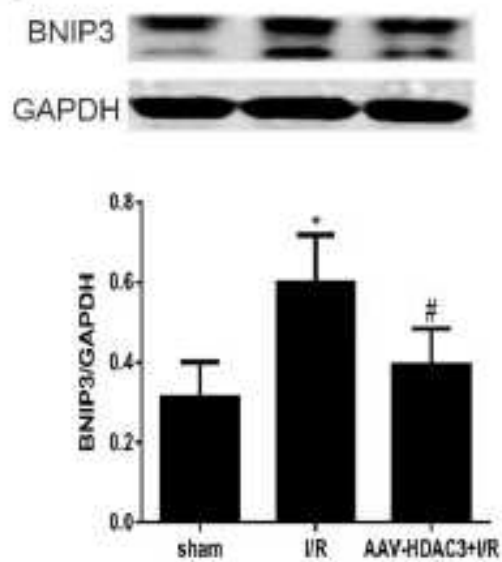

b

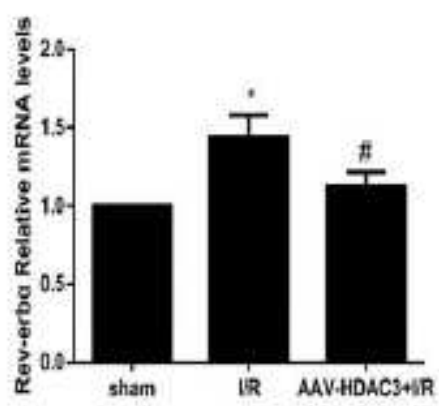

e
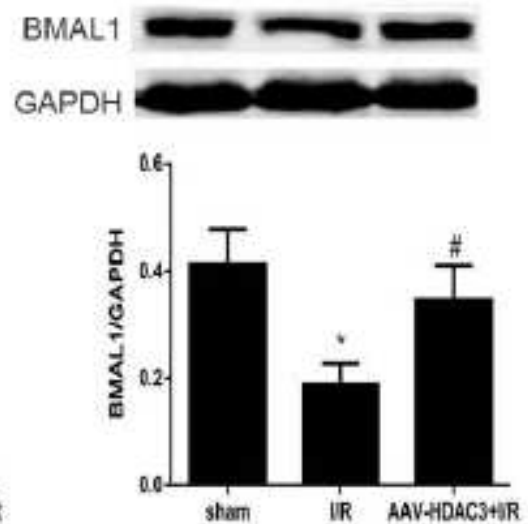

h
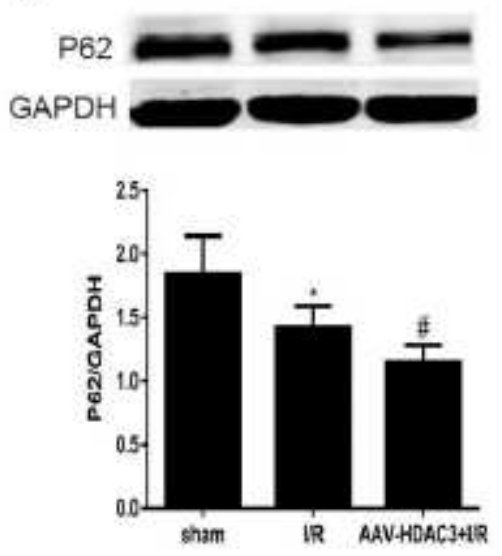

C

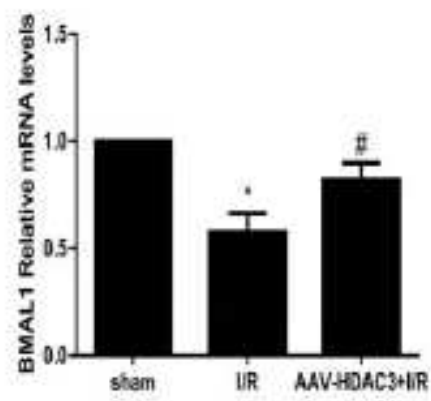

f

CIEBPB

GAPDH

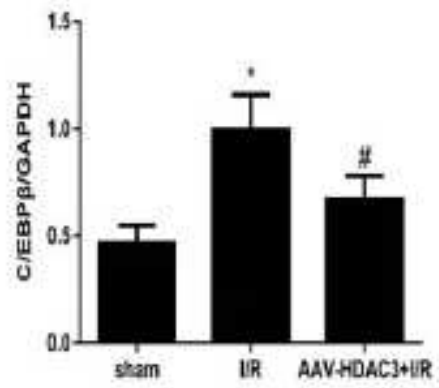

i
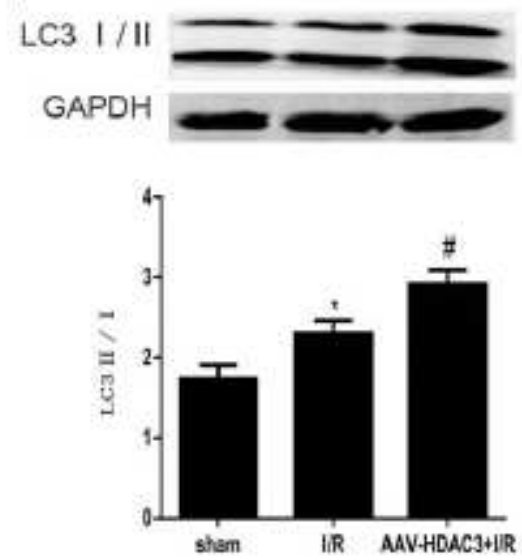

\section{Figure 8}

Knockdown expression of HDAC3 attenuated diabetic MI/RI by regulating Rev-erba/BMAL1 circadian oscillations to recover mitophagy level. (a-c) The mRNA expressions of HDAC3 (a), Rev-erba (b) and BMAL1 (c) were analysed by qPCR. (d-i) The protein levels of Rev-erba (d), BMAL1 (e), C/EBPß (f), BNIP3(g), P62 (h) and LC3区/ (i) were measured by western blotting in non-diabetes and diabetes with or without I/R insult. $\mathrm{n}=6$ per group. ${ }^{*} \mathrm{P}<0.05$ versus sham; $\# \mathrm{P}<0.05$ versus I/R. I/R, ischemia/reperfusion; AAV-HDAC3+I/R, HBAAV9-r-HDAC3 shRNA1-GFP injection+ ischemia/reperfusion. 
a

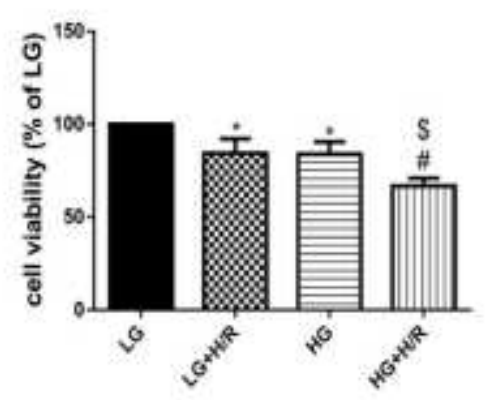

C
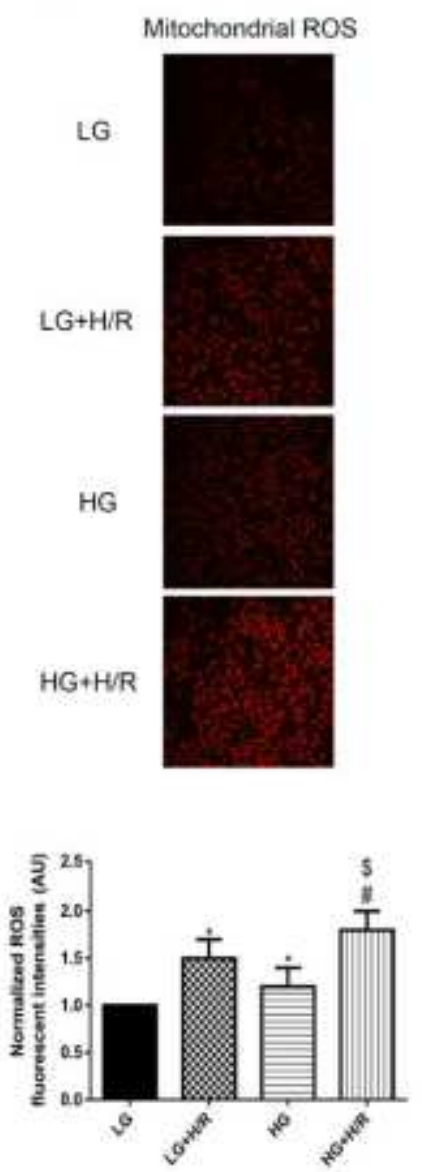

d
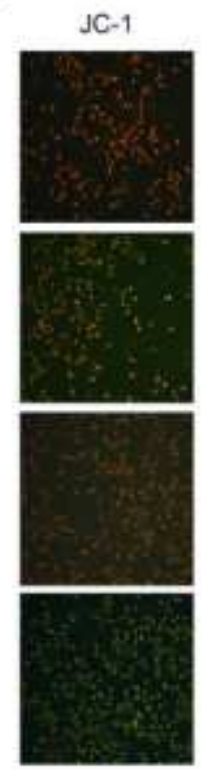

b

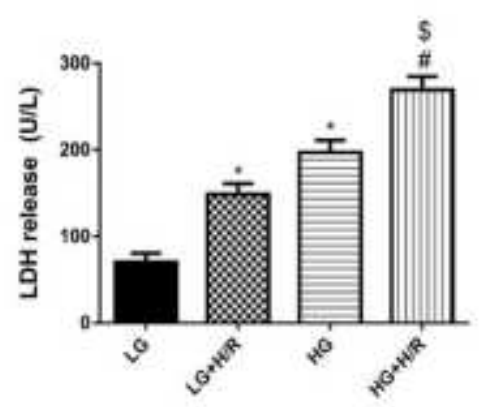

e

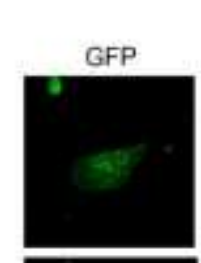

Autophagic flux

MRFP
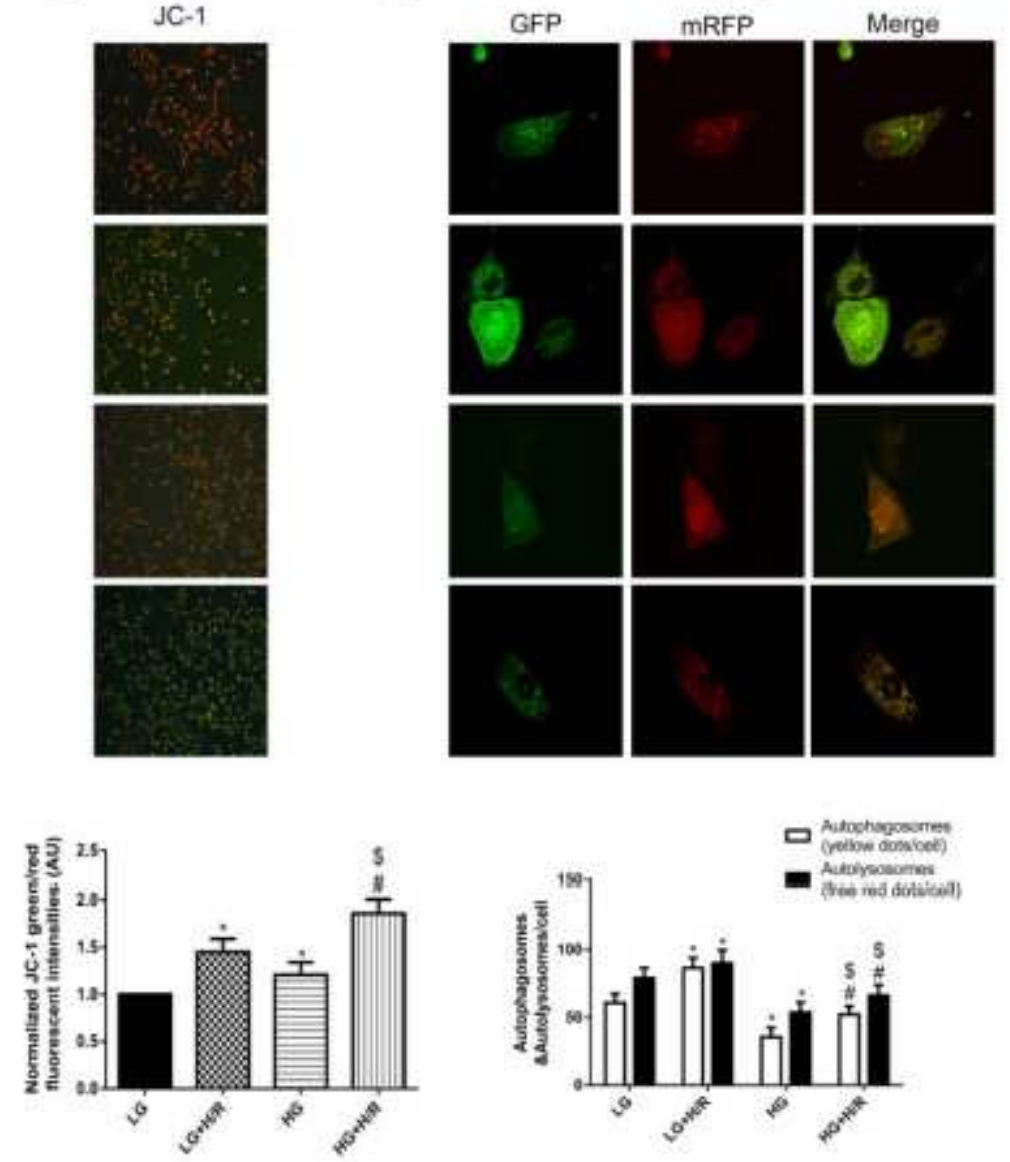

\section{Figure 9}

HG aggravates H/R injury of cardiomyocytes by reducing autophagic flow levels and mitochondrial function. (a) Cell viability was analysed by CCK-8 kit. (b) Serum level of LDH was detected by ELISA kit. (c-d) Mitochondrial ROS (c) and JC-1 (d) were detected to analyse mitochondrial function. Scale bar: 100 $\mu \mathrm{m}$. (e) Tandem fluorescent mRFP-GFP-LC3 adenovirus was used to detect the autophagic flux. Scale bar: $20 \mu \mathrm{m}$. $\mathrm{n}=6$ per group. *P<0.05 LG; \#P<0.05 versus HG; $\$ \mathrm{P}<0.05$ versus LG+H/R. LG, low glucose; LG+H/R, low glucose+hypoxia/reoxygenation; $\mathrm{HG}$, high glucose; $\mathrm{HG}+\mathrm{H} / \mathrm{R}$, high glucose+hypoxia/reoxygenation. 
a

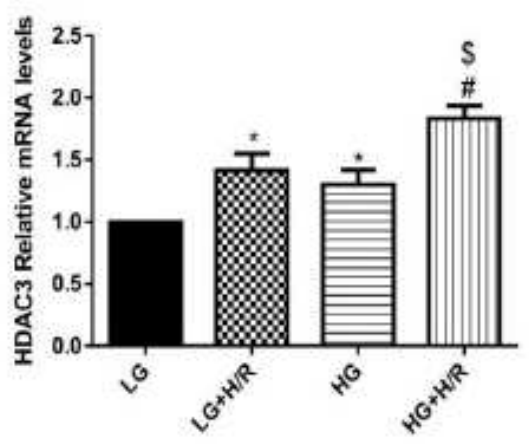

d
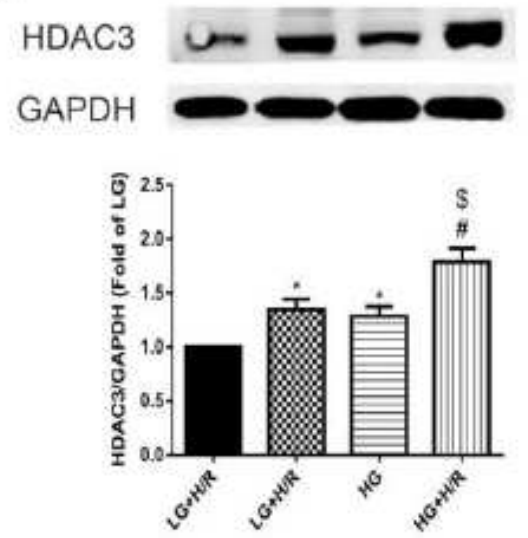

g
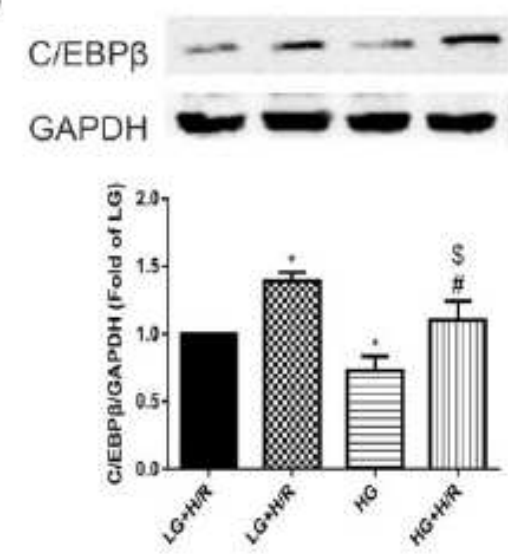

b

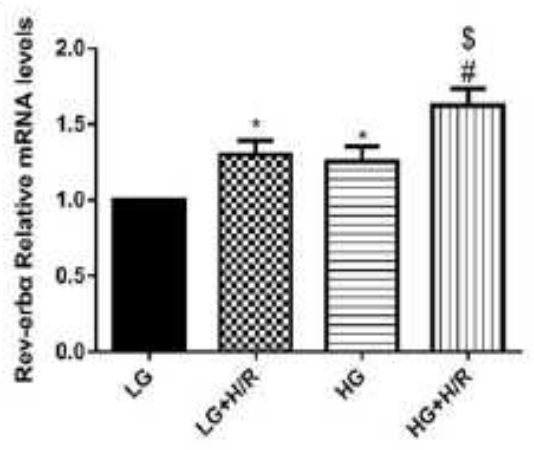

e
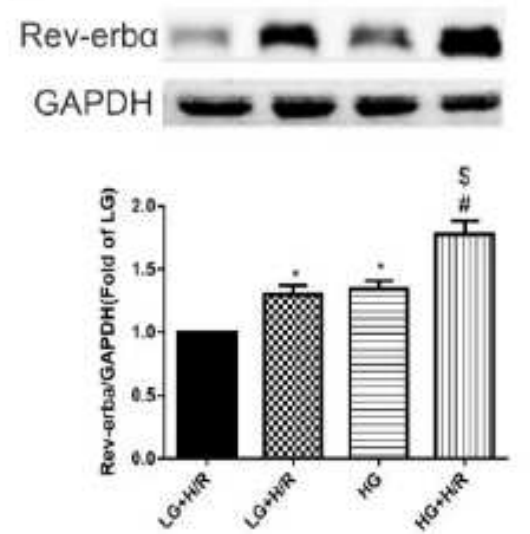

h
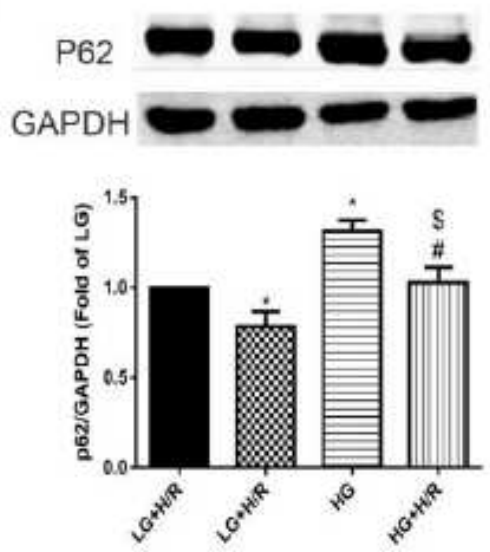

C

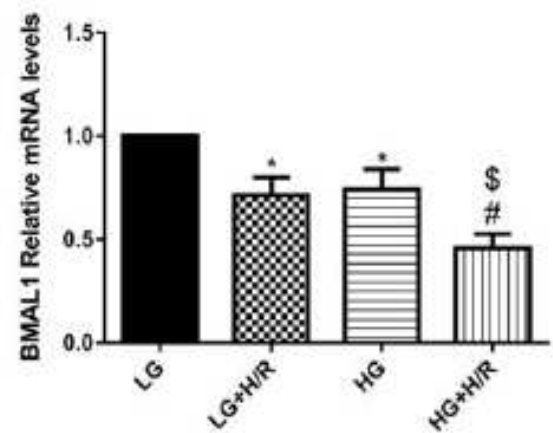

f
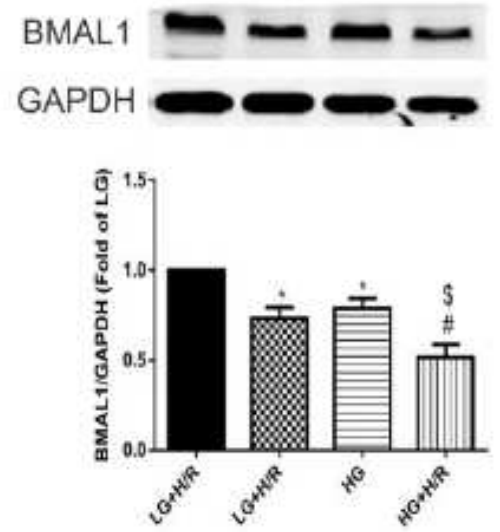

i
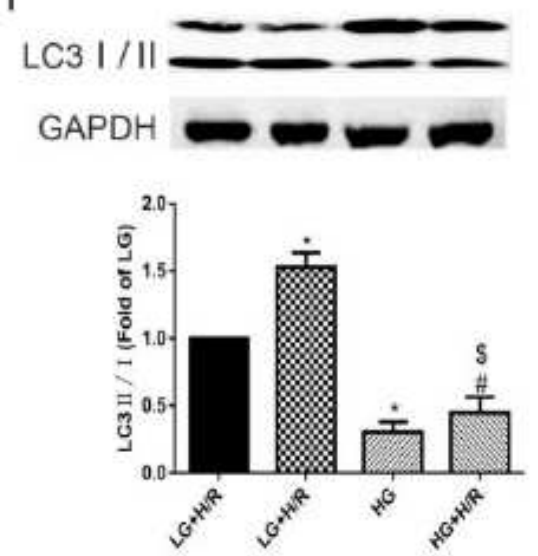

Figure 10

Increased expression of HDAC3 mediates Rev-erba/BMAL1 in high glucose to increase the vulnerability of H/R by inhibiting mitophagy of neonatal rat cardiomyocytes. (a-c) The mRNA levels of HDAC3 (a), Reverba (b) and BMAL1 (c) were analysed by qPCR. (d-i) The protein levels of HDAC3 (d), Rev-erba (e), BMAL1 (f), C/EBPß (g), P62 (h) and LC3区/ $(\mathrm{i})$ were analysed by western blotting in the cultured neonatal rat cardiomyocytes. $n=6$ per group. * $P<0.05$ LG; \#P<0.05 versus $H G$; $\$ P<0.05$ versus $L G+H / R$. LG, low glucose; LG+H/R, low glucose+hypoxia/reoxygenation; $H G$, high glucose; $H G+H / R$, high glucose+hypoxia/reoxygenation. 
a

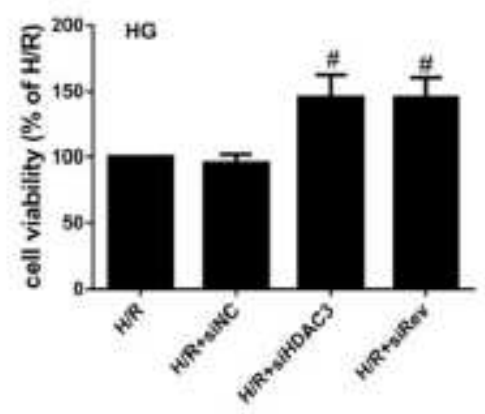

C
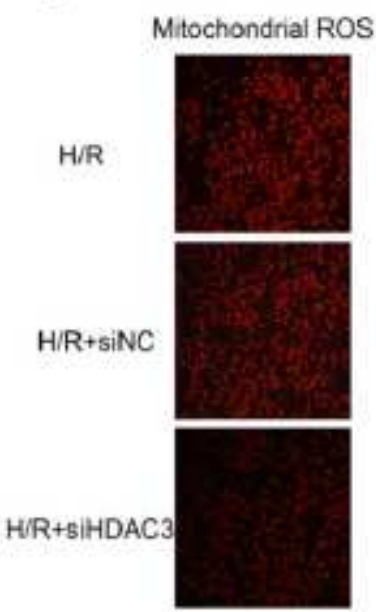

H/R+siRev
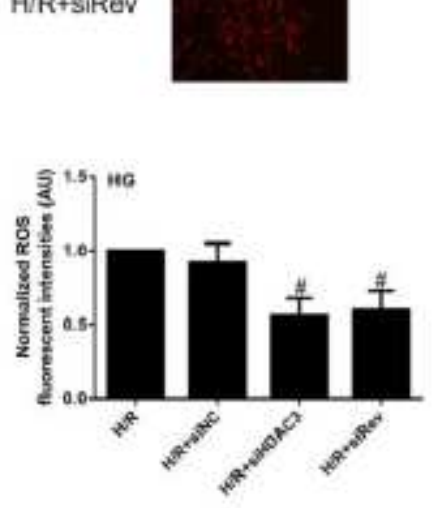

d
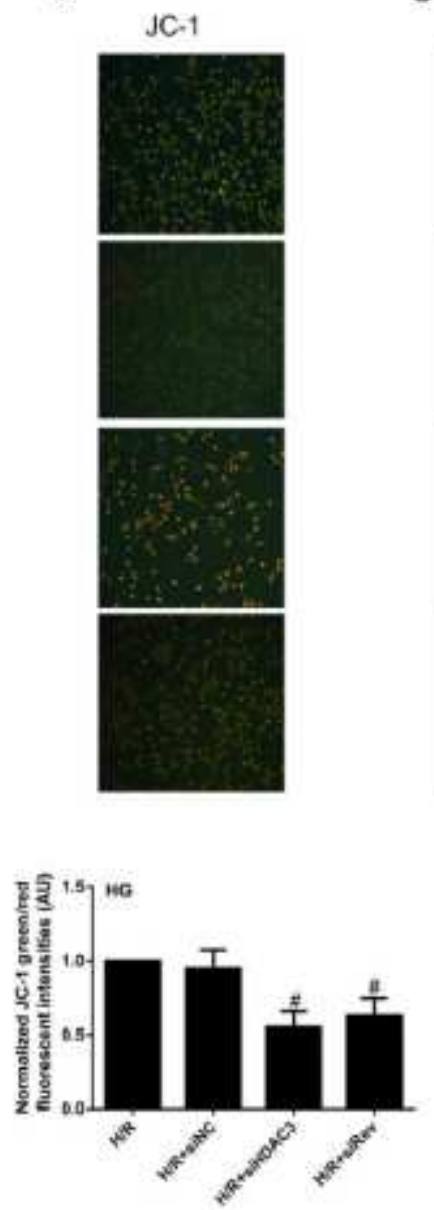

e b
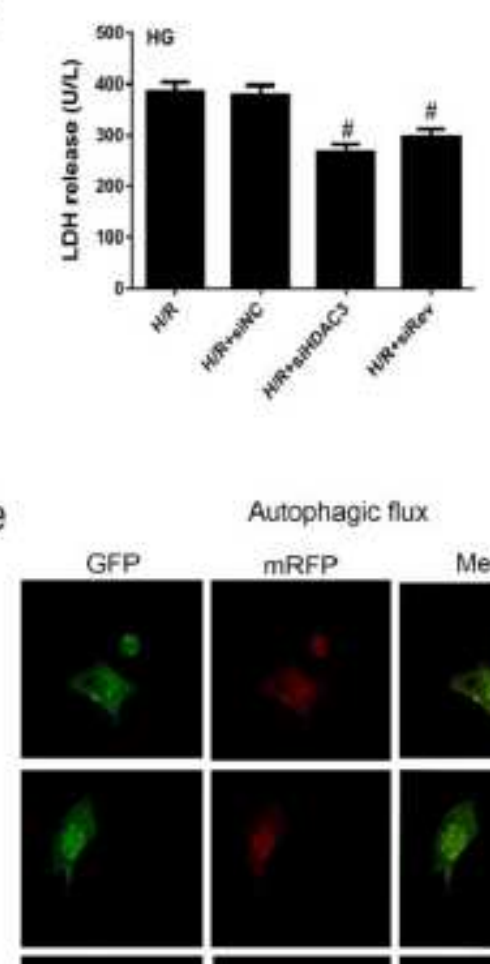

Autophagic flux

mRFP

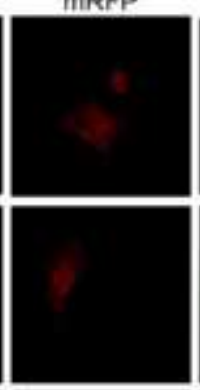

Merge
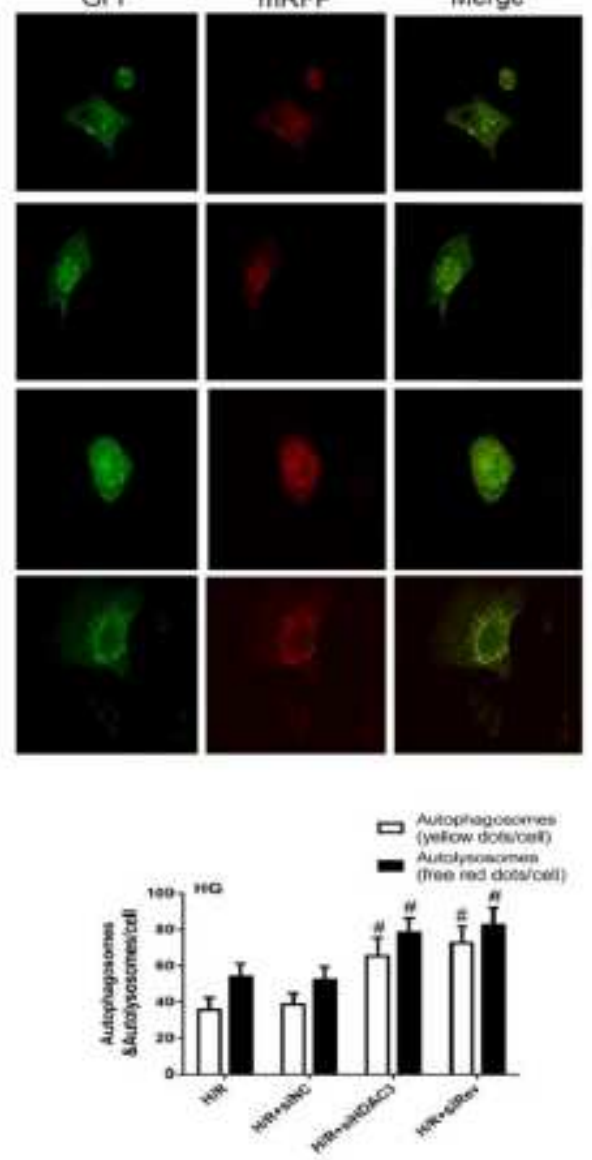

Figure 11

Knockdown HDAC3/Rev-erba expression could attenuated H/R injury of neonatal rat cardiomyocytes by increasing autophagic flow levels and mitochondrial function. (a) Cell viability was analysed by CCK-8 kit. (b) The serum level of LDH was analysed by ELISA kit. (c-d) Mitochondrial ROS (c) and JC-1 (d) were detected to anlyse mitochondrial function. Scale bar: $100 \mu \mathrm{m}$. (e) Tandem fluorescent mRFP-GFP-LC3 adenovirus was used to detect autophagic flux. Scale bar: $20 \mu \mathrm{m}$. $n=6$ per group. $\# P<0.05$ versus $H / R$. $\mathrm{H} / \mathrm{R}$, hypoxia/reoxygenation; $\mathrm{H} / \mathrm{R}+\mathrm{siNC}$, hypoxia/reoxygenation+normal control siRNA; H/R+siHDAC3, hypoxia/reoxygenation+ HDAC3 siRNA; H/R+siRev, hypoxia/reoxygenation+Rev-erba siRNA. 
a

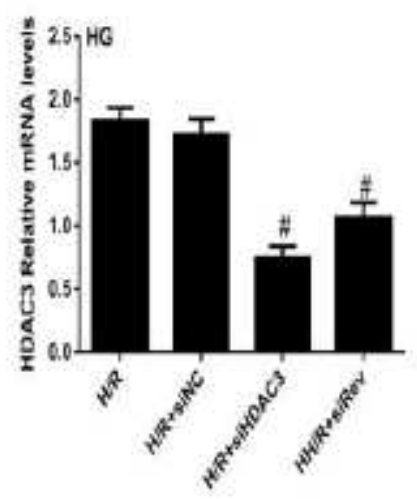

d
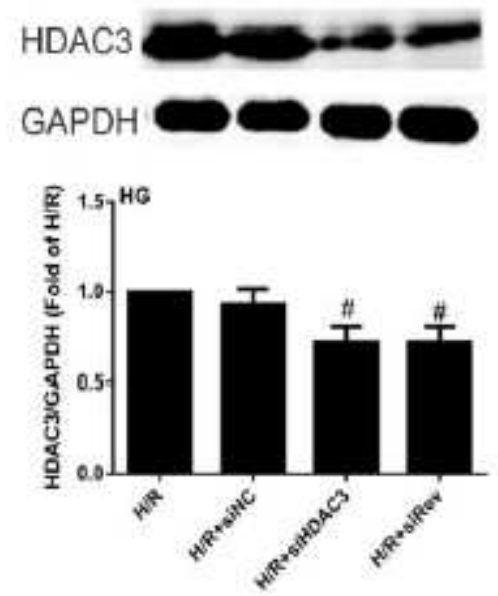

g
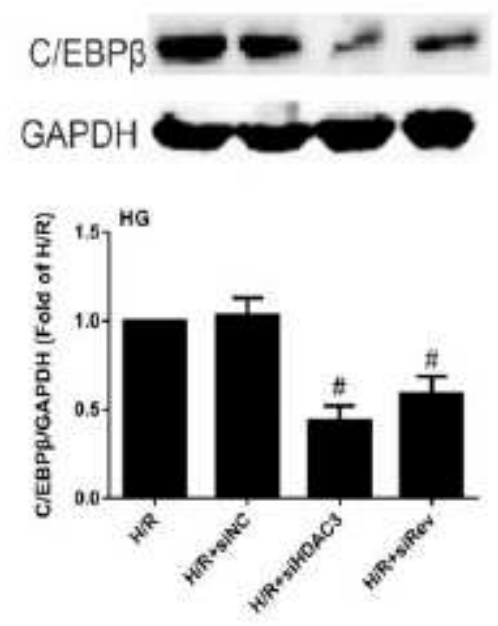

b

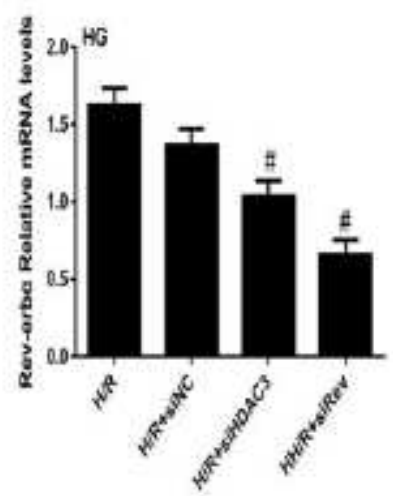

e
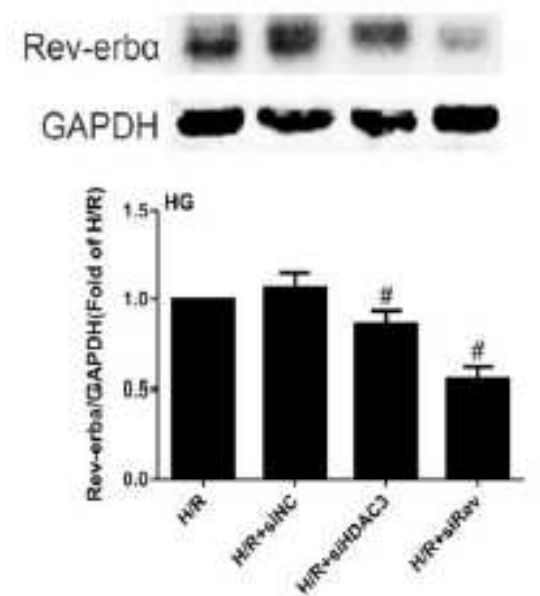

h
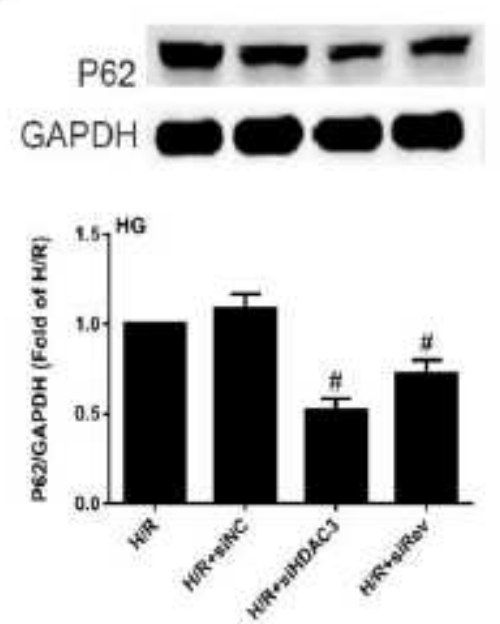

C

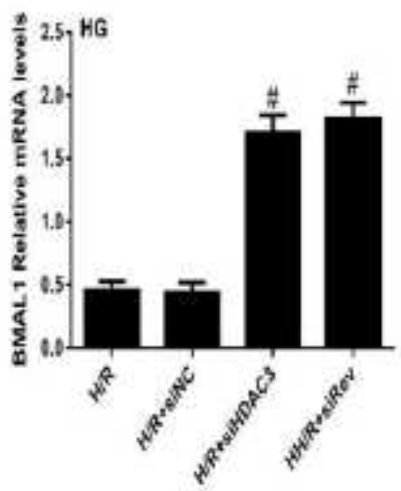

f
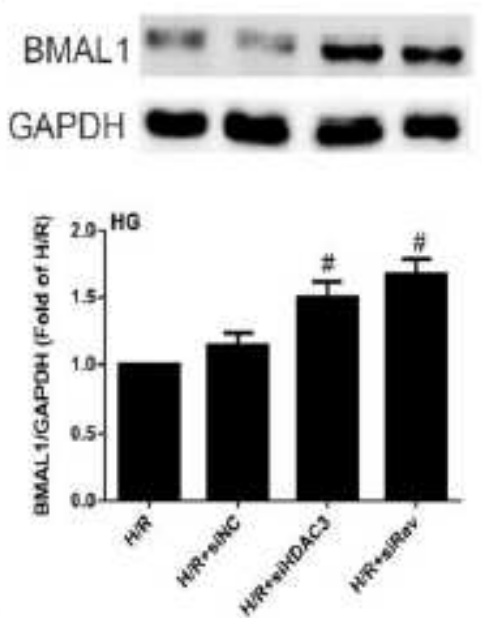

i
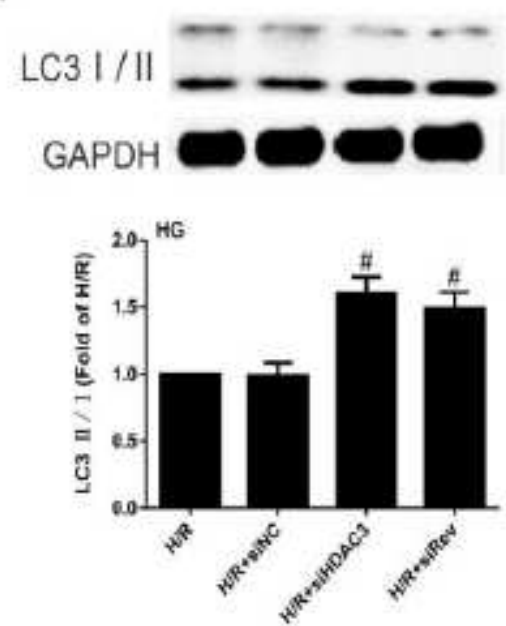

Figure 12

Knockdown HDAC3/Rev-erba expression could attenuated H/R injury of neonatal rat cardiomyocytes by up-regulating BMAL1 expression to activate mitophagy of neonatal rat cardiomyocytes under HG condition. (a-c) The mRNA levels of HDAC3 (a), Rev-erba (b) and BMAL1 (c) were analysed by qPCR. (d-i) The protein levels of HDAC3 (d), Rev-erba (e), BMAL1 (f), C/EBPß (g), P62 (h) and LC3区/ه (i) were analysed by western blotting in the cultured neonatal rat cardiomyocytes. $n=6$ per group. $\# P<0.05$ versus 
$H / R$. H/R, hypoxia/reoxygenation; H/R+siNC, hypoxia/reoxygenation+normal control siRNA; $\mathrm{H} / \mathrm{R}+$ siHDAC3, hypoxia/reoxygenation+ HDAC3 siRNA; H/R+siRev, hypoxia/reoxygenation+Rev-erba SiRNA. 